Portland State University

PDXScholar

\title{
Spacetime Numerical Techniques for the Wave and Schrödinger Equations
}

Paulina Ester Sepùlveda Salas

Portland State University

Follow this and additional works at: https://pdxscholar.library.pdx.edu/open_access_etds

Part of the Applied Mathematics Commons

Let us know how access to this document benefits you.

\section{Recommended Citation}

Sepùlveda Salas, Paulina Ester, "Spacetime Numerical Techniques for the Wave and Schrödinger Equations" (2018). Dissertations and Theses. Paper 4206.

https://doi.org/10.15760/etd.6094

This Dissertation is brought to you for free and open access. It has been accepted for inclusion in Dissertations and Theses by an authorized administrator of PDXScholar. Please contact us if we can make this document more accessible: pdxscholar@pdx.edu. 
Spacetime Numerical Techniques for the Wave and Schrödinger Equations

by

Paulina Ester Sepúlveda Salas

A dissertation submitted in fulfillment of the requirements for the degree of

Doctor of Philosophy

in

Mathematical Sciences

Dissertation Committee:

Jay Gopalakrishnan, Chair

Panayot Vassilevski

Jeffrey Ovall

Johnny Guzmán

Erik Sánchez

Portland State University

2018 
(C) 2018 Paulina Ester Sepúlveda Salas 


\begin{abstract}
The most common tool for solving spacetime problems using finite elements is based on semidiscretization: discretizing in space by a finite element method and then advancing in time by a numerical scheme. Contrary to this standard procedure, in this dissertation we consider formulations where time is another coordinate of the domain. Therefore, spacetime problems can be studied as boundary value problems, where initial conditions are considered as part of the spacetime boundary conditions.

When seeking solutions to these problems, it is natural to ask what are the correct spaces of functions to choose, to obtain wellposedness. This motivates the study of an abstract theory for unbounded partial differential operators associated with a general boundary value problem on a bounded domain. A framework for choosing the spaces is introduced, and conditions for the solvability of weak formulations are provided. We apply this framework to study wave problems on tents and to study wellposed discontinuous Petrov-Galerkin (DPG) formulations for the Schrödinger and wave equations. Several numerical issues are also discussed.
\end{abstract}




\section{Acknowledgements}

First of all, I thank my advisor, Prof. Jay Gopalakrishnan, for guiding me in the process of obtaining my doctorate degree, for his support, time and for all the opportunities he gave me to travel to conferences and math meetings. It has been an honor to work with him during these years.

I also thank Prof. Ignacio Muga Urquiza, professor at Pontificia Universidad Católica de Valparaíso. He encouraged me to continue my studies when I was carrying through my bachelors degree. He also suggested that I continue my studies in the USA and helped me in the process to reach higher levels of education. He has always motivated me to pursue my academic goals.

Thanks to Dr. David George, Research Mathematician at USGS Cascades Volcano Observatory, with whom I worked with in my first internship. Thanks to Prof. Panayot Vassilevski, with whom I had the pleasure of working during my last internship in Lawrence Livermore National Laboratory, and all the people in the lab that I met during my stay in Livermore. Thanks to all the members of my committee for being part of this process as well.

I also thank Prof. Joachim Schöberl, who was willing to explain to me how the finite element sofware, NGSolve, is implemented during his visit to Portland State University on Summer 2016. Thanks to his initial help and with the subsequent guidance of Prof. Gopalakrishnan, we were able to implement a repository for solving linear elasticity with weakly symmetric stress tensors using the NGSolve library. 
Thanks to the Fariborz Maseeh Department of Mathematics and especially to the Portland State University community for the enriching Maseeh Mathematics \& Statistics Colloquia, and for the opportunities to present part of my work in the Applied \& Computational Mathematical Seminar, in the SIAM Chapter meetings and in the group meetings led by Prof. Jay Gopalakrishnan. I also thank the Eugene Enneking Doctoral Fellowship for the financial support on my first years at Portland State University. Thanks to Department of Mathematics for the wonderful opportunity to be a math instructor during my last year of studies.

Thanks to Prof. Michael Karkulik, who is currently a professor at Universidad Federico Santa Maria in Chile, and Dr. Kirill Voronin, research assistant at Portland State University, for the many talks about math. Thanks to Dr. Chak Shing Lee, postdoc at Lawrence Livermore National Laboratory, for his guidance in software implementations using the finite element library MFEM and thanks to many other professors with whom I had the pleasure to talk about mathematics and physics during my stay at Portland State University.

This work would not have been possible without the support of CONICYT Becas Chile 72130448, NSF grant (DMS-1318916), the AFOSR grant (FA9550-12-1-0484). The numerical experimentation was facilitated by the resources of Portland Institute for Computational Sciences (PICS) acquared under NFS grant DMS-1624776.

Finally, I thank God, my loving family, my friends, all the people that supported me in my studies and my stay at Portland State University. All these people motivated me to give my best to achieve my goals and dreams and made my stay here a great experience. Special thanks my friends from Chile, Spain, Poland, Korea, Japan, and the USA, whom I consider part of my family. 


\section{Table of Contents}

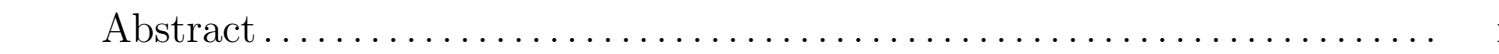

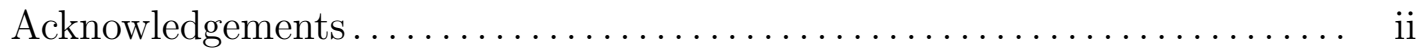

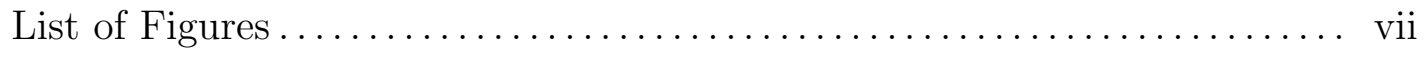

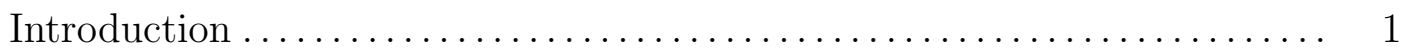

$1 \quad$ Abstract ultraweak formulation ........................... 5

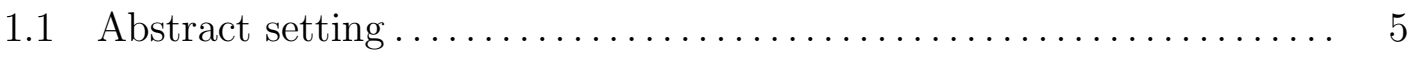

1.2 The graph spaces and boundary operator $\ldots \ldots \ldots \ldots \ldots \ldots \ldots \ldots$

1.3 Selecting domains of differential operators $\ldots \ldots \ldots \ldots \ldots \ldots \ldots \ldots$

$1.4 \quad$ A mesh dependent weak formulation $\ldots \ldots \ldots \ldots \ldots \ldots \ldots \ldots \ldots \ldots \ldots$

\begin{tabular}{|l}
\hline $1.4 .1 \quad$ A proof of wellposedness $\ldots \ldots \ldots \ldots \ldots \ldots \ldots \ldots \ldots \ldots \ldots \ldots$ \\
15
\end{tabular}

1.4 .2 An alternate proof of wellposedness ................ 17

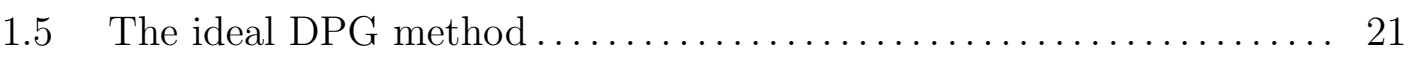

1.5.1 An Example of wellposed problem under this setting ....... 22

1.6 Defining the $V$ and $V^{*}$ spaces from spaces of smooth functions ...... 24

$2 \quad$ Hyperbolic systems in one space dimension $\ldots \ldots \ldots \ldots \ldots \ldots \ldots \ldots \ldots \ldots$

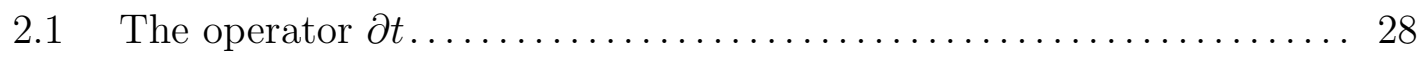

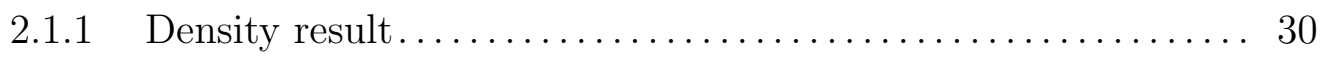

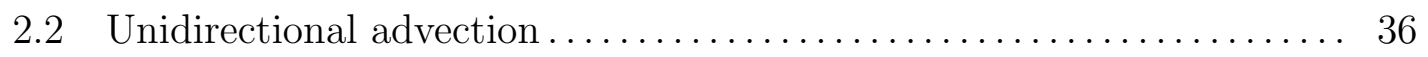

$2.3 \quad$ Advection operator on tent-shaped domains $\ldots \ldots \ldots \ldots \ldots \ldots \ldots$. 41 
$2.4 \quad$ Linear symmetric hyperbolic system $\ldots \ldots \ldots \ldots \ldots \ldots \ldots \ldots \ldots \ldots$

$3 \quad$ A tent pitching scheme for the wave equation $\ldots \ldots \ldots \ldots \ldots \ldots \ldots \ldots \ldots$

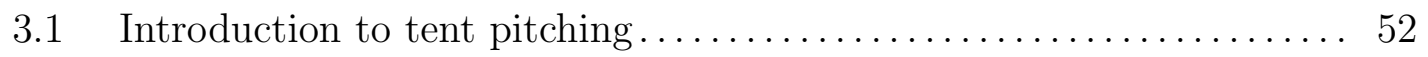

3.2 Model problem: wave equation $\ldots \ldots \ldots \ldots \ldots \ldots \ldots \ldots \ldots \ldots \ldots \ldots \ldots$

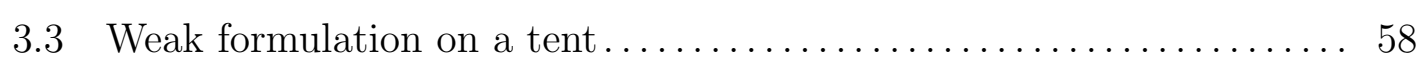

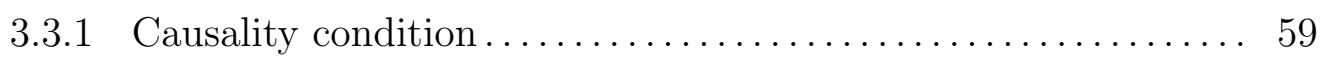

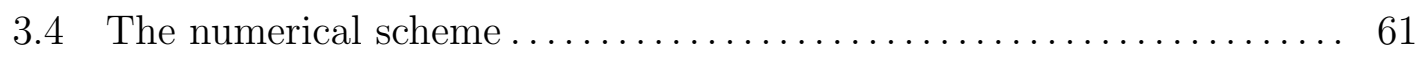

3.4.1 Conforming discretization on a tent $\ldots \ldots \ldots \ldots \ldots \ldots \ldots \ldots$. 61

3.4 .2 Advancing in time by tent pitching $\ldots \ldots \ldots \ldots \ldots \ldots \ldots \ldots$

3.4 .3 Propagation formula $\ldots \ldots \ldots \ldots \ldots \ldots \ldots \ldots \ldots \ldots \ldots \ldots \ldots \ldots$

3.4 .4 Error analysis on uniform grids $\ldots \ldots \ldots \ldots \ldots \ldots \ldots \ldots \ldots \ldots$

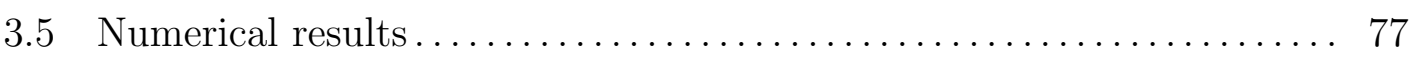

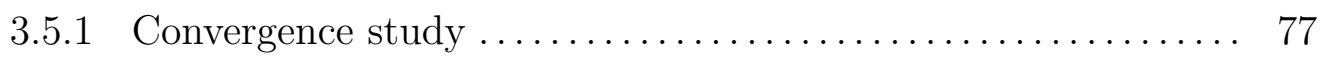

3.5.2 Material interfaces and other boundary conditions .......... 79

$4 \quad$ A spacetime DPG method for the Schrodinger equation $\ldots \ldots \ldots \ldots \ldots \ldots$. 84

$4.1 \quad$ Introduction to the problem $\ldots \ldots \ldots \ldots \ldots \ldots \ldots \ldots \ldots \ldots \ldots \ldots \ldots \ldots$

$4.2 \quad$ Wellposed formulation $\ldots \ldots \ldots \ldots \ldots \ldots \ldots \ldots \ldots \ldots \ldots \ldots \ldots \ldots \ldots \ldots$

4.2 .1 Strong formulation $\ldots \ldots \ldots \ldots \ldots \ldots \ldots \ldots \ldots \ldots \ldots \ldots \ldots \ldots \ldots$

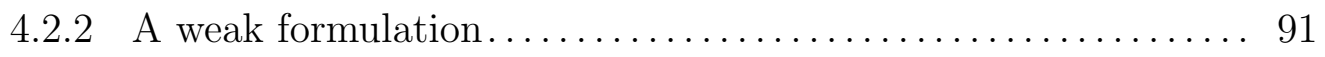

$4.2 .3 \quad$ Verification of the density assumption $\ldots \ldots \ldots \ldots \ldots \ldots \ldots 2$

4.3 Error Estimates for the ideal DPG method ................. 96

$5 \quad$ A spacetime DPG method for the first order wave equation $\ldots \ldots \ldots \ldots \ldots$. 102

5.1 Model problem: The transient wave problem $\ldots \ldots \ldots \ldots \ldots \ldots \ldots \ldots$. 102

5.1 .1 The formal wave operator $\ldots \ldots \ldots \ldots \ldots \ldots \ldots \ldots \ldots \ldots \ldots \ldots$

5.1 .2 The unbounded wave operator $\ldots \ldots \ldots \ldots \ldots \ldots \ldots \ldots \ldots \ldots \ldots$ 


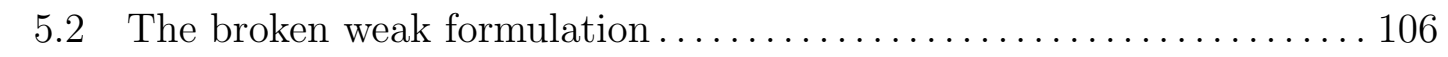

$5.3 \quad$ Verification of the density condition $\ldots \ldots \ldots \ldots \ldots \ldots \ldots \ldots \ldots \ldots \ldots, \ldots \ldots$

5.4 The method and its error estimates ........................... 116

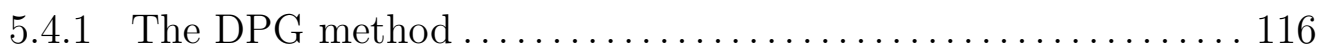

$5.5 \quad$ Implementation and numerical results $\ldots \ldots \ldots \ldots \ldots \ldots \ldots \ldots \ldots \ldots \ldots$

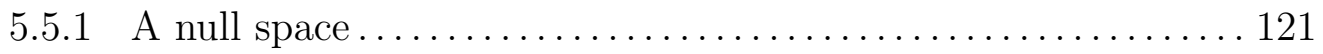

5.5 .2 Techniques to solve despite the null space $\ldots \ldots \ldots \ldots \ldots \ldots 123$

5.5.3 Convergence rates in two-dimensional spacetime ............ 124

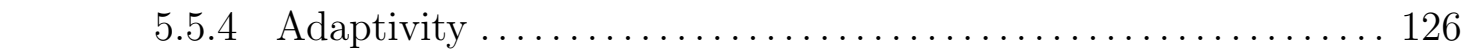

5.5 .5 Convergence rates in three-dimensional spacetime........... 127

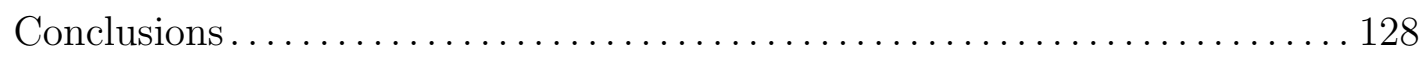

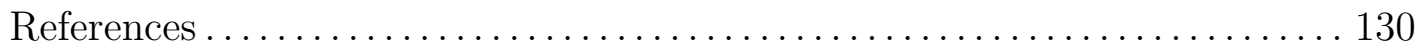




\section{List of Figures}

Figure $1.1 \quad$ Rates of convergence for $u$ in the $L^{2}$-norm using a triangular mesh. 24

Figure $2.1 \quad$ Reference domain $K \subset \mathbb{R}^{2} \quad 28$

Figure $2.2 \quad$ Spacetime tent in $\mathbb{R}^{d}$ with $d=3$.

Figure $2.3 \quad$ On the left is a tent $K$ with $A=\partial_{t}+0.5 \partial_{x}$ that satisfies (2.3.3).

On the right is $\hat{K}$ obtained after applying the map in the proof of

Theorem 2.3 .2 with mapped over operator $\hat{A}=\partial_{\hat{t}}$.

Figure $3.1 \quad$ Tent pitching mesh (read row by row) 53

Figure $3.2 \quad$ Mapping $\Omega$ to $\hat{\Omega}_{1}$ using the $H_{1}$ transformation. $\quad 56$

$\begin{array}{lll}\text { Figure } 3.3 \quad \text { Three types of tents } & 60\end{array}$

$\begin{array}{lll}\text { Figure } 3.4 \quad \text { Type I: } h_{r}>0, h_{l}>0 & 71\end{array}$

Figure $3.5 \quad \zeta(x, t)$ basis function for tent of Type I 73

$\begin{array}{lll}\text { Figure } 3.6 \quad \text { The stencil } & 75\end{array}$

$\begin{array}{lll}\text { Figure } 3.7 & \text { Comparison with CTCS scheme } & 78\end{array}$

Figure $3.8 \quad$ Wave propagation through an impedance-matched interface 81

Figure $3.9 \quad$ Case of reflected and transmitted waves 83

Figure $4.1 \quad$ Real part of $U_{M}(x, t)$ with different values of $M$.

Figure $4.2 \quad$ Schematic of the spacetime domain 89

\begin{tabular}{lll|l|l|}
\hline Figure & Extension and translation in the proof of Theorem & 4.2 .7
\end{tabular} 
Figure $4.4 \quad$ Degrees of freedom in the $p=3$ (left) and $p=5$ (right) cases.

Figure $5.1 \quad$ Left: Extended domains $Q_{1}$ and $Q_{2}$ when $\Omega \subseteq \mathbb{R}^{3}$. Right: Translation by $\delta$ in the $t$ direction.

Figure $5.2 \quad$ Example of a spacetime shape function $z_{j}$ in the kernel

Figure $5.3 \quad$ Iterates from the adaptive algorithm. Numerical pressure $\mu$ is shown for $p=3$. Time axis is vertical.

Figure $5.4 \quad$ Convergence rates for $\left\|u-u_{h}\right\|$ on triangular meshes using Technique 1 on the left, and on rectangular meshes using Technique 1 on the right

Figure $5.5 \quad$ Convergence rates for $\left\|u-u_{h}\right\|$ on tretrahedral meshes using Technique 2 on the left, and on hexahedral meshes using Technique 1 on the right 


\section{Introduction}

A wide variety of physical problems can be described using partial differential equations involving time and space. For example, the wave equation can arise in acoustics, electromagnetism, elasticity problems, and other areas. Many numerical methods already exist to solve these time-dependent problems. The most widely used technique is based on semidiscretization: discretizing in space and advancing in time by a time stepping scheme. One of the major drawbacks of these methods when considering hyperbolic problems is that they impose a global Courant-Friedrichs-Lewy (CFL) condition (see [14]), which makes local mesh-refinement a difficult task. One alternative to deal with this problem is to consider time as another coordinate and formulate a spacetime boundary value problem. The advantages of this approach include a natural way of treating moving boundary conditions, easy spacetime adaptivity, possibility to obtain convergence even under limited spacetime regularity and exploitation of parallelism without causality constraints, among others (see for example [16], [51], [52], 64] and references therein).

The first ideas to advance a numerical solution in time by local operations in space time regions were explored in $[\mathbf{5 3}, 4]$. Recurrence relations on multiple slabs of rectangular spacetime elements were considered in [44]. These ideas were generalized to non-regular spacetime elements for beams and plates in [5]. Closest in ancestry to one of the methods we consider in this dissertation is found in [56], where it was called explicit spacetime elements. 
The spacetime discontinuous Galerkin (SDG) method was announced almost at the same time in [48, [56] and continues to see active development over the years in engineering applications [49, 55, 71].

An important numerical technique that we shall highlight in this dissertation was first introduced in two papers [33, 50]. There we can find the first tent pitching ideas that were brought into the numerical analysis community. A tent pitching algorithm, which was first introduced in [67], generates an unstructured mesh for the spacetime domain using an advancing-front scheme. Recent advances include in [39], where the mapped tent pitching (MTP) method has been introduced in higher dimensions. This technique transforms the equations within a spacetime tent to a domain where space and time are separable. In this dissertation, we are interested in the study of the properties of functions of Hilbert spaces endowed with a graph norm, and their traces in tent-shaped domains, where inflow and outflow part of the boundary meet. Moreover, we are interested in the design of an explicit finite element method of the tent-shaped type whose discrete spaces conform to the weak continuity properties of the space. We also highlight other numerical techniques that consider spacetime domains (see [68]).

In this dissertation we are also interested in studying the feasibility of using the discontinuous Petrov-Galerkin (DPG) method for the spacetime Schrödinger and wave equations. The DPG method was introduced in its current form in [17] and makes use of different trial and test spaces, where optimal test functions are considered using broken test norms that allow their local calculation and guarantees the stability of the method (see [17, 19, 22, 21]). The DPG method has been successfully applied to a wide variety of problems such as second order elliptic problems [18], convective phenomena, 15, 17, 23], elasticity [6, 10, 38, 45], etc. Among the desirable properties of the DPG method are mesh-independent stability, inheritance of discrete stability 
from the wellposedness of the undiscretized problem, and a built-in error estimator that exhibits good pre-asymptotic mesh-independent stability properties. Applications of the DPG method for spacetime problems have already been computationally studied in [28 for the transient parabolic partial differential equations and [27] for the time-dependent convection-diffusion equation. We also note that a scheme that combines DPG spatial discretization with backward Euler time stepping for the heat equation has been analyzed in [34].

One of the contributions of the present dissertation is to provide a general theory for the wellposedness of ultraweak formulations for boundary value poblems. Spacetime formulations that consider time as part of the domain can be studied in the context of boundary value problems, where initial and boundary conditions are incorporated into boundary conditions of the spacetime domain. In order to seek solutions in suitable spaces, allowing source terms in a non-regular space, we study the unbounded differential operators related to the problem. We state, in a general context, what are the necessary conditions for the weak formulations to be wellposed. Moreover, we show a strategy to avoid the explicit use of traces, which are difficult to define in non-standard graph spaces. We are interested in applying these numerical techniques for the Schrödinger and wave equation.

This dissertation consists of five chapters. In Chapter 1, we introduce an abstract setting for a general boundary value problem, define non-standard Hilbert spaces on graph norms related to unbounded operators that include the boundary conditions of the problems in a weak sense, and show the sufficient conditions for a broken weak formulation to be wellposed. In Chapter 2 we introduce a theory for the wellposedness of local problems in tent-shaped domains for linear hyperbolic problems, and prove trace lemmas for these operators in a weak sense. In Chapter 3 we present an explicit finite element discretization of the tent pitching type for solving the wave equation, 
and study the conditions to obtain a conforming method, based on the weak continuity property of the traces of functions in the lowest order case. In Chapter 4, we apply the theory developed in Chapter 1 to explore a weak formulation and a numerical technique for the second order Schrödinger equation with Dirichlet boundary conditions. From a theoretical point of view, the study of the Schrödinger equation gives an important example of a partial differential equation that should not be reformulated as a first order system. This is the reason for going beyond traditional Friedrichs systems [30] in Chapter 1. In Chapter 5, we study a suitable DPG formulation for the wave equation, following the approach laid out in our study of the DPG method for the Schrödinger equation. One important contribution of this chapter is the density result of smooth functions with boundary conditions for the space where we seek the solutions in any $d$-dimensional hyperrectangle. The key results presented here can also be found in the following journal articles [40, [16] and [37, which were written during this doctoral study. 


\section{Abstract ultraweak formulation}

In this chapter, we are interested in deriving and identifying the sufficient conditions for the wellposedness of a mesh-dependent weak formulation of a boundary value problem involving a general partial differential operator. To motivate this framework, we will study and apply this abstract framework to the Laplace problem throughout this chapter.

\subsection{Abstract setting}

Let $\Omega \subseteq \mathbb{R}^{d}$ be a bounded non-empty open set in $d \geq 1$ dimensions, and let the operator $A: L^{2}(\Omega)^{m} \rightarrow L^{2}(\Omega)^{l}$ be a (unbounded) linear operator such that the $i$ th component of $A u$ is

$$
[A u]_{i}=\sum_{j=1}^{m} \sum_{|\alpha| \leq k} \partial^{\alpha}\left(a_{i j \alpha} u_{j}\right),
$$

where $l, m, k$ are integers, $a_{i j \alpha}: \Omega \rightarrow \mathbb{C}$ are complex valued functions for all $i=1, \ldots l$, $j=1, \ldots, m$, and all multi-indices $\alpha=\left(\alpha_{1}, \ldots \alpha_{d}\right)$ whose length is $|\alpha|=\alpha_{1}+\cdots+\alpha_{d} \leq$ $k$. As usual, $\partial^{\alpha}=\partial_{1}^{\alpha_{1}} \cdots \partial_{d}^{\alpha_{d}}, \mathcal{D}(\bar{\Omega})$ denote the restrictions of functions from $\mathcal{D}\left(\mathbb{R}^{d}\right)$ to $\Omega$. Here, $\mathcal{D}\left(\mathbb{R}^{d}\right)$ denotes the space of $C^{\infty}$ scalar-valued functions with compact support, and $\mathcal{D}^{\prime}(\Omega)$ denotes the space of distributions. $L^{2}(\Omega)^{m}$ represents the space of vector functions where each of their $m$-components is in $L^{2}(\Omega)$. The formal adjoint of $A$ is given by $A^{*}$, and its $j$ th component is given by

$$
\left[A^{*} v\right]_{j}=\sum_{i=1}^{l} \sum_{|\alpha| \leq k}(-1)^{|\alpha|} \overline{a_{j i \alpha}} \partial^{\alpha} v_{j} .
$$


We assume that the coefficients $a_{i j \alpha}$ are such that

$$
A^{*} u \in \mathcal{D}^{\prime}(\Omega)^{m} \quad \text { for all } u \in L^{2}(\Omega)^{l} .
$$

EXAMPLE 1.1.1. As motivation, we will study and apply this setting to the Laplace problem throughout this chapter. We are interested in finding $u$ such that

$$
\begin{aligned}
-\operatorname{div}(\nabla u)=f & \text { on } \Omega, \\
u=0 & \text { on } \partial \Omega,
\end{aligned}
$$

with $f \in L^{2}(\Omega)$. Here, $\Omega$ is a Lipschitz domain and $\partial \Omega$ denotes its boundary. Setting $\sigma=\nabla u$, we can rewrite the second order problem 1.1 .2 and obtain the following equivalent first order system. Now we seek $\sigma$ and $u$ such that

$$
\begin{aligned}
\sigma-\nabla u=0 & \text { on } \Omega, \\
-\operatorname{div}(\sigma)=f & \text { on } \Omega, \\
u=0 & \text { on } \partial \Omega .
\end{aligned}
$$

Introducing a new vector-unknown $w=[\sigma, u]^{T}$, the diagonal identity operator $I_{d \times d}$, and defining the unbounded operator $A: L^{2}(\Omega)^{d+1} \rightarrow L^{2}(\Omega)^{d+1}$ such that

$$
A w=\left[\begin{array}{cc}
I_{d \times d} & -\nabla \\
-\operatorname{div} & 0
\end{array}\right] w
$$

we can rewrite the equations $1.1 .3 \mathrm{a}$ into the equivalent problem of finding a vector function $w$ such that

$$
A w=\left[\begin{array}{l}
0 \\
f
\end{array}\right] .
$$


In this example, the operator $A$ is of the form $(\mathrm{A}$-a when setting $m=l=d+1$ and $k=1$. Since its formal adjoint is given by $A^{*} w=\left[\begin{array}{cc}I_{d \times d} & \nabla \\ \operatorname{div} & 0\end{array}\right] w$, then condition (A-b) is also satisfied.

\subsection{The graph spaces and boundary operator}

In this section, we introduce spaces of functions related to the operators defined in $\mathrm{A}-\mathrm{a}$ and $\mathrm{A}-\mathrm{b})$. For any non-empty open subset $S \subseteq \Omega$, we define the space

$$
W(S)=\left\{u \in L^{2}(S)^{m}: A u \in L^{2}(S)^{l}\right\}
$$

normed by $\|u\|_{W(S)}=\left(\|u\|_{S}^{2}+\|A u\|_{S}^{2}\right)^{1 / 2}$, and the space

$$
W^{*}(S)=\left\{u \in L^{2}(S)^{l}: A^{*} u \in L^{2}(S)^{m}\right\}
$$

normed by $\|u\|_{W^{*}(S)}=\left(\|u\|_{S}^{2}+\left\|A^{*} u\right\|_{S}^{2}\right)^{1 / 2}$. Here and throughout, $(\cdot, \cdot)_{S}$ and $\|$. $\|_{S}$ denotes the inner product and the norm, respectively, in $L^{2}(S)$ or its Cartesian products. To simplify the notation, we abbreviate $W=W(\Omega), W^{*}=W^{*}(\Omega)$. Clearly these are inner product spaces.

Lemma 1.2.1. The spaces $W(S)$ and $W^{*}(S)$ are Hilbert spaces.

Proof. Since the proofs for $W(S)$ and $W^{*}(S)$ are similar, we only show the first. Suppose $u_{n}$ is a Cauchy sequence in $W(S)$. Then $u_{n}$ is Cauchy in $L^{2}(S)^{m}$ and $A u_{n}$ is Cauchy in $L^{2}(S)^{l}$. Hence there is a $u \in L^{2}(S)^{m}$ and $f \in L^{2}(S)^{l}$ such that $\left\|u-u_{n}\right\|_{S} \rightarrow 0$ and $\left\|f-A u_{n}\right\|_{S} \rightarrow 0$. We will show that $u$ is in $W(S)$.

Let $\phi \in \mathcal{D}(S)^{l}$. For each $w \in L^{2}(S)^{m}$, the distributional action of $A w$ on $\bar{\phi}$, denoted by $\langle A w, \phi\rangle_{\mathcal{D}(S)^{l}}$, equals $\left(w, A^{*} \phi\right)_{S}$ for all $\phi$ in $\mathcal{D}(S)^{l}$. Thus $A w \in L^{2}(\Omega)^{l}$. To 
complete the proof, note that

$$
\langle A u, \phi\rangle_{\mathcal{D}(S)^{l}}=\left(u, A^{*} \phi\right)_{S}=\lim _{n \rightarrow \infty}\left(u_{n}, A^{*} \phi\right)_{S}=\lim _{n \rightarrow \infty}\left(A u_{n}, \phi\right)_{S}=(f, \phi)_{S}
$$

for all $\phi$ in $\mathcal{D}(S)^{l}$. Hence $A u=f$ and $u$ is in $W(S)$.

Next, define bounded linear operators $D_{S}: W(S) \rightarrow W^{*}(S)^{\prime}$ and $D_{S}^{*}: W^{*}(S) \rightarrow$ $W(S)^{\prime}$ by

$$
\begin{aligned}
\left\langle D_{S} u, v\right\rangle_{W^{*}(S)} & =(A u, v)_{S}-\left(u, A^{*} v\right)_{S}, \\
\left\langle D_{S}^{*} v, u\right\rangle_{W(S)} & =\left(A^{*} v, u\right)_{S}-(v, A u)_{S},
\end{aligned}
$$

for all $u \in W(S)$ and $v \in W^{*}(S)$. When $S=\Omega$, we abbreviate $D_{S}$ and $D_{S}^{*}$ to $D$ and $D^{*}$, respectively. Here and in the remainder, we use $\langle\cdot, \cdot\rangle_{X}$ to denote the action of a linear functional in $X^{\prime}$ on an element of $X$.

ExAmple 1.2.2. For the system considered in Example 1.1.1), $W=W^{*}=$ $H(\operatorname{div}, \Omega) \times H^{1}(\Omega)$. Here, $H(\operatorname{div}, \Omega)=\left\{\sigma \in L^{2}(\Omega)^{d}: \operatorname{div} \sigma \in L^{2}(\Omega)\right\}$, and $H^{1}(\Omega)=$ $\left\{u \in L^{2}(\Omega): \nabla u \in L^{2}(\Omega)^{d}\right\}$. Using the well-known trace theorems for $H^{1}(\Omega)$ and $H(\operatorname{div}, \Omega)$ and Green identities, (see [7]) the identity

$$
\langle D u, v\rangle_{W}=(A u, v)-\left(u, A^{*} v\right)=-\left\langle\hat{u}_{\sigma_{n}}, \hat{v}_{\mu}\right\rangle_{H^{1 / 2}(\partial \Omega)}-\left\langle\hat{v}_{\sigma_{n}}, \hat{u}_{\mu}\right\rangle_{H^{1 / 2}(\partial \Omega)}
$$

holds for any $u=\left(u_{\sigma}, u_{\mu}\right)$ and $v=\left(v_{\sigma}, v_{\mu}\right) \in W$, where $\hat{u}_{\sigma_{n}}, \hat{v}_{\sigma_{n}}$ represent the wellknown normal traces of $u_{\sigma}, v_{\sigma}$ respectively, while $\hat{u}_{\mu}$ and $\hat{v}_{\mu}$ represent the traces of $u_{\mu}$ and $v_{\mu}$ respectively. Here and throughout, $n$ represents the unit outward normal vector to the respective domain. 


\subsection{Selecting domains of differential operators}

Next, we consider $A: \operatorname{dom}(A) \subset L^{2}(\Omega)^{m} \rightarrow L^{2}(\Omega)^{l}$ as an unbounded linear operator whose domain (denoted by $\operatorname{dom}(A))$ is chosen such that

$$
\mathcal{D}(\Omega)^{m} \subseteq \operatorname{dom}(A) .
$$

This implies that $A$ is a densely defined operator, and therefore there exists a unique adjoint operator whose domain is given by

$$
\left\{v \in L^{2}(\Omega)^{l}: \exists \ell \in L^{2}(\Omega)^{m} \text { such that }(A u, v)=(u, \ell) \text {, for all } u \in \operatorname{dom}(A)\right\}
$$

We take a moment to explain why we have chosen the notation $A^{*}$ to denote the formal adjoint, which in the literature is used to define the the adjoint operator in the sense of unbounded operators. For reasons of clarity, we will very shortly assume that the adjoint operator is denoted by $A^{\prime}$ and show that they are equal on the domain of the adjoint operator.

Identifying the dual of (Cartesian products of) $L^{2}(\Omega)$ with itself, the domain of $A^{\prime}$ is given by (1.3.1) and it satisfies $(A u, v)_{\Omega}=\left(u, A^{\prime} v\right)_{\Omega}$ for all $u \in \operatorname{dom}(A)$ and for all $v$ belonging to the set defined in (1.3.1). If we choose $u \in \mathcal{D}(\Omega)^{m}$, we can conclude that the distribution $A^{*} s=A^{\prime} s$, for all $s \in \operatorname{dom}\left(A^{\prime}\right)$. Hence, since the adjoint operator is a closed linear operator uniquely defined after setting the domain of $A$ on the set (1.3.1) (see [7]), we keep the notation of $A^{*}$ to denote both: the differential operator in (1.1.1) and the formal adjoint of the unbounded operator $A$.

When $\operatorname{dom}(A)$ is endowed with the topology of $W(\Omega)$, we call it $V$, i.e., although $V$ and $\operatorname{dom}(A)$ coincide as sets, $V$ has the topology of $W(\Omega)$ and $\operatorname{dom}(A)$ has the topology of $L^{2}(\Omega)^{m}$. Similarly, $\operatorname{dom}\left(A^{*}\right)$ is called $V^{*}$ when it is endowed with the topology of $W^{*}(\Omega)$. For the next result, recall that the left annihilator of any subspace 
$R$ of the dual space $X^{\prime}$ of any Banach space $X$ is defined by ${ }^{\perp} R=\left\{w \in X:\left\langle s^{\prime}, w\right\rangle_{X}=\right.$ 0 for all $\left.s^{\prime} \in R\right\}$.

LEMma 1.3.1. In the setting of (A-a), A-b and (A-c), we have $V^{*}={ }^{\perp} D(V)$.

Proof. Let us first prove that $V^{*} \subseteq D(V)$. According to the (above-mentioned) definition of $\operatorname{dom}\left(A^{*}\right)$, for any $v \in \operatorname{dom}\left(A^{*}\right)=V^{*}$, there is an $\ell \in L^{2}(\Omega)^{l}$ such that

$$
(A u, v)_{\Omega}=(u, \ell)_{\Omega}, \quad \forall u \in V
$$

Due to $\mathrm{A}-\mathrm{C}$, we may choose $u$ in $\mathcal{D}(\Omega)^{m}$. By $\mathrm{A}-\mathrm{b}, A^{*} v$ is a distribution and by (1.3.2 this distribution is in $L^{2}(\Omega)^{m}$ and equals $\ell$. In particular, $v$ is in $W^{*}(\Omega)$. Hence (1.2.3) is applicable, and in combination with 1.3 .2 yields $\langle D u, v\rangle_{W^{*}(\Omega)}=$ $(A u, v)_{\Omega}-(u, \ell)_{\Omega}=0$ for all $u \in V$. Hence $v \in{ }^{\perp} D(V)$ and we have proved that $V^{*} \subseteq{ }^{\perp} D(V)$. The reverse containment is also easy to prove. Indeed, if $w \in{ }^{\perp} D(V)$, then $w \in W^{*}$ then $\langle D u, w\rangle=0$ for all $u \in \operatorname{dom}(A)$. Thus, setting $\ell=A^{*} w$, we obtain $(A u, w)_{\Omega}-(u, \ell)_{\Omega}=0$ for all $u \in V$. Then $w \in V^{*}$.

ExAmple 1.3.2. Let $u, v$ be in $W=H(\operatorname{div}, \Omega) \times H^{1}(\Omega)$ in our model problem 1.1.2), where $u=\left(u_{\sigma}, u_{\mu}\right)$ and $v=\left(v_{\sigma}, v_{\mu}\right)$. 1.2.5) We define $V=H(\operatorname{div}, \Omega) \times$ $H_{0}^{1}(\Omega)$. It is well known that $\mathrm{A}$-c holds, and that $V$ is a subspace of $L^{2}(\Omega)^{d+1}$. Therefore, the adjoint operator is uniquely defined on

$$
\begin{aligned}
\operatorname{dom}\left(A^{*}\right)=\left\{v=\left(v_{\sigma}, v_{\mu}\right) \in L^{2}(\Omega)^{d+1}: \exists \ell \in L^{2}(\Omega)^{d+1}\right. \text { such that } \\
\left.(A u, v)=\left(\left[\begin{array}{c}
u_{\sigma}-\nabla u_{\mu} \\
-\operatorname{div} u_{\sigma}
\end{array}\right], v\right)=(u, \ell), \quad \forall u \in V\right\} .
\end{aligned}
$$

Since the distribution $A^{*} v \in L^{2}(\Omega)^{d+1}$ for all $v \in \operatorname{dom}\left(A^{*}\right)$, then $\operatorname{dom}\left(A^{*}\right)$ is a subspace of $W^{*}$, and defined as $V^{*}$, as a space with the topology of $W^{*}$. 
From 1.2 .5 and 1.3 .3$)$ we have

$$
V^{*}=H(\operatorname{div}, \Omega) \times H_{0}^{1}(\Omega)
$$

Thus, $V=V^{*}$.

\subsection{A mesh dependent weak formulation}

We are interested in the boundary value problem of finding $u$ satisfying

$$
A u=f, \quad u-g \in V
$$

given $f \in L^{2}(\Omega)^{l}$ and $g \in W$. Homogeneous boundary conditions are incorporated in $V$. In this way, spacetime problems with non-homogeneous spacetime boundary conditions can be abstracted into this form. When $l=m$, it makes sense to talk about the solvability of the adjoint problem: finding $u \in W^{*}$, such that $u-g^{*} \in V^{*}$, with $g^{*} \in W^{*}$, satisfying $A^{*} u=f$.

Consider the scenario where $\Omega$ is partitioned into a mesh $\Omega_{h}$ of finitely many open elements $K$ such that $\bar{\Omega}=\cup_{K \in \Omega_{h}} \bar{K}$. Here the index $h$ denotes $\max _{K \in \Omega_{h}} \operatorname{diam}(K)$. Recall $D_{K}$ and $D_{K}^{*}$ by replacing $S$ by $K$ in 1.2 .3 and (1.2.4).

Additionally, set

$$
W_{h}=\prod_{K \in \Omega_{h}} W(K), \quad\left(W_{h}^{*}\right)^{\prime}=\prod_{K \in \Omega_{h}} W^{*}(K)^{\prime}
$$

The spaces $W_{h}^{*}$ and $W_{h}^{\prime}$ are defined similarly. The component on an element $K$ of functions in such product spaces are indicated by placing $K$ as a subscript, e.g., for any $w$ in $W_{h}$, the component of $w$ on element $K$ is denoted by $w_{K}$. Let $D_{h}: W_{h} \rightarrow\left(W_{h}^{*}\right)^{\prime}$ 
be the continuous linear operator defined by

$$
\left\langle D_{h} w, v\right\rangle_{W_{h}^{*}}=\sum_{K \in \Omega_{h}}\left\langle D_{K} w_{K}, v_{K}\right\rangle_{W^{*}(K)}
$$

for all $w \in W_{h}$ and $v \in W_{h}^{*}$. To simplify notation, we abbreviate $\left\langle D_{h} w, v\right\rangle_{W_{h}^{*}}$ to $\left\langle D_{h} w, v\right\rangle_{h}$, i.e., duality pairing in $W_{h}^{*}$ is simply denoted by $\langle\cdot, \cdot\rangle_{h}$. For any $w \in W_{h}$, we denote by $A_{h} w$ the function obtained by applying $A$ to $w_{K}$, element by element, for all $K \in \Omega_{h}$. The resulting function $A_{h} w$ is an element of $\Pi_{K \in \Omega_{h}} L^{2}(K)^{l}$, which is identified to be the same as $L^{2}(\Omega)^{l}$. The operator $A_{h}^{*}: W_{h}^{*} \rightarrow L^{2}(\Omega)^{m}$ is defined similarly. Thus

$$
\left\langle D_{h} w, v\right\rangle_{h}=\left(A_{h} w, v\right)_{\Omega}-\left(w, A_{h}^{*} v\right)_{\Omega}
$$

for all $w \in W_{h}$ and $v \in W_{h}^{*}$.

Lemma 1.4.1. For all $w \in W$ and $v \in W^{*}$, we have $\left\langle D_{h} w, v\right\rangle_{h}=\langle D w, v\rangle_{W^{*}}$.

Proof. If $w \in W$ and $v \in W^{*}$, then $A_{h} w=A w$ and $A_{h}^{*} v=A^{*} v$. Using this in 1.4 .3$),\left\langle D_{h} w, v\right\rangle_{h}=(A w, v)_{\Omega}-\left(w, A^{*} v\right)_{\Omega}=\langle D w, v\rangle_{W^{*}}$ whenever $w$ is in $W$ and $v$ is in $W^{*}$.

To derive the mesh-dependent weak formulation, multiply 1.4 .1 by a test function $v \in W_{h}^{*}$ and apply the definition of $D_{K}$. Summing over all $K \in \Omega_{h}$, we obtain $\left(u, A_{h}^{*} v\right)_{\Omega}+\left\langle D_{h}(u-g), v\right\rangle_{h}=(f, v)_{\Omega}-\langle D g, v\rangle_{W^{*}}$ for all $v$ in $W_{h}^{*}$. Let

$$
Q=\left\{r \in\left(W_{h}^{*}\right)^{\prime}: \text { there is a } u \in V \text { such that } r=D_{h} u\right\} \text {. }
$$

Setting $D_{h} u$ to be a new unknown $q$ in $Q$ and defining the sesquilinear form $b((u, q), v)=\left(u, A_{h}^{*} v\right)_{\Omega}+\langle q, v\rangle_{h}$ on $\left(L^{2}(\Omega)^{m} \times Q\right) \times W_{h}^{*}$, we have thus derived the following weak formulation. 
Problem 1.4.2. (Ultraweak formulation) Given any $F \in\left(W_{h}^{*}\right)^{\prime}$, find $u \in L^{2}(\Omega)^{m}$ and $q \in Q$ such that

$$
b((u, q), v)=F(v), \text { for all } v \in W_{h}^{*} .
$$

ExAmPLE 1.4.3. For our model problem introduced in (1.1.2), an exact characterization of $Q$ is possible. This is derived by the well known trace theorems of $H^{1}(\Omega)$ and $H(\operatorname{div}, \Omega)$ as mentioned in [38]. Namely,

$$
Q \equiv H^{-1 / 2}\left(\partial \Omega_{h}\right) \times H_{0}^{1 / 2}(\partial \Omega)
$$

where

$$
\begin{aligned}
& H^{-1 / 2}\left(\partial \Omega_{h}\right)=\left\{\eta \in \prod_{K} H^{-1 / 2}(\partial K): \exists q \in H(\operatorname{div}, \Omega) \text { such that }\left.\eta\right|_{\partial K}=\left.q \cdot n\right|_{\partial K}, \forall K \in \Omega_{h}\right\}, \\
& H^{1 / 2_{0}}\left(\partial \Omega_{h}\right)=\left\{u \in \prod_{K} H^{1 / 2}(\partial K): \exists w \in H_{0}^{1}(\Omega) \text { such that }\left.u\right|_{\partial K}=\left.w\right|_{\partial K}, \forall K \in \Omega_{h}\right\} .
\end{aligned}
$$

We define

$$
Q^{*}=\left\{r \in\left(W_{h}\right)^{\prime}: \text { there is a } v \in V^{*} \text { such that } r=D_{h}^{*} v^{*}\right\} \text {. }
$$

In the same way, setting $D_{h}^{*} v=\tilde{q}$ and defining the sesquilinear form $b^{*}((v, \tilde{q}), u)=$ $\left(v, A_{h} u\right)_{\Omega}+\langle\tilde{q}, u\rangle_{W_{h}}$ on $\left(L^{2}(\Omega)^{l} \times Q\right) \times W_{h}$, the adjoint weak formulation derived from the dual problem reads as

Problem 1.4.4. (Adjoint ultraweak formulation when $l=m$ ) Given any $F^{*} \in$ $\left(W_{h}\right)^{\prime}$, find $u \in L^{2}(\Omega)^{l}$ and $\tilde{q} \in Q^{*}$ such that

$$
\left(u, A_{h} v\right)_{\Omega}-\langle\tilde{q}, v\rangle_{h}=F^{*}(v), \quad \forall v \in W_{h} .
$$


THEOREM 1.4.5. In the setting of (A-a), (A-b) and (A-c), suppose

$$
\begin{aligned}
& V={ }^{\perp} D^{*}\left(V^{*}\right), \text { and } \\
& A: V \rightarrow L^{2}(\Omega)^{l} \text { is a bijection. }
\end{aligned}
$$

Then, Problem 1.4.2 is well posed. Moreover, if $F(v)=(f, v)_{\Omega}+\left\langle D_{h} g, v\right\rangle_{h}$ for some $f \in L^{2}(\Omega)^{l}$ and $g \in W$, then the solution $(u, q)$ of Problem 1.4.2 is such that $u-g \in V$ and $q=D_{h} u$.

Theorem 1.4.6. Analogously, if (A-a), A-b and (A-c) hold, and

$$
\begin{aligned}
& V={ }^{\perp} D^{*}\left(V^{*}\right), \text { and } \\
& A^{*}: V^{*} \rightarrow L^{2}(\Omega)^{m} \text { is a bijection, }
\end{aligned}
$$

then, Problem 1.4.4 is wellposed. Moreover, if $F(v)=(f, v)_{\Omega}$ for some $f \in L^{2}(\Omega)^{m}$, then the unique solution $u$ of Problem 1.4.2 is in $V$, solves (1.4.1), and satisfies $\tilde{q}=D_{h}^{*} u$.

Before we prove Theorem 1.4.5, we must note how our assumptions allow a natural topology on $Q$ and $Q^{*}$. Specifically, 1.4 .9 implies that $V$ is a closed subspace of $W$. It is also a closed subspace of $W_{h}$, since $W$ is continuously embedded in $W_{h}$. The same embedding also shows that the restriction of $D_{h}$ to $V$, denoted by $D_{h, V}: V \rightarrow\left(W_{h}^{*}\right)^{\prime}$, is continuous. Note that $Q$ is the range of $D_{h, V}$. For any $r$ in $Q$, we use $D_{h, V}^{-1}(\{r\})$ to denote the pre-image of $r$, i.e., set of all $v \in V$ such that $r=D_{h} v$. The continuity of $D_{h, V}$ implies that $D_{h, V}^{-1}(\{0\})$ is a closed subspace of $V$. Hence

$$
\|q\|_{Q}=\inf _{v \in D_{h, V}^{-1}(\{q\})}\|v\|_{W}
$$


is a norm on $Q$. This quotient norm makes $Q$ complete. Similarly,

$$
\|q\|_{Q^{*}}=\inf _{v \in D_{h, V^{*}}^{*-1}(\{q\})}\|v\|_{W^{*}}
$$

is a norm with which $Q^{*}$ is complete. The wellposedness result of Theorem 1.4 .5 is to be understood with $Q$ endowed with this norm.

1.4.1. A proof of wellposedness. We now give a proof of Theorem 1.4.5. Recall that the right annihilator of any subspace $S \subseteq X$ is defined by $S^{\perp}=\left\{w^{\prime} \in W^{\prime}\right.$ : $\left\langle w^{\prime}, s\right\rangle_{W}=0$ for all $\left.s \in S\right\}$. The next lemma is used below to prove uniqueness.

LEMMA 1.4.7. If 1.4.9 holds, then $D_{h} V \subseteq\left(V^{*}\right)^{\perp}$.

Proof. Let $u \in V \subseteq W_{h}$. Then for any $v \in V^{*}$, the functional $D_{h} u \in\left(W_{h}^{*}\right)^{\prime}$ satisfies $\left\langle D_{h} u, v\right\rangle_{h}=\langle D u, v\rangle_{W^{*}}$ by Lemma 1.4.1. But $\langle D u, v\rangle_{W^{*}}=-\overline{\left\langle D^{*} v, u\right\rangle_{W}}=0$ since (1.4.9) shows that $u \in{ }^{\perp} D^{*}\left(V^{*}\right)$. Hence $D_{h} u \in\left(V^{*}\right)^{\perp}$.

Proof of Theorem 1.4.5. We verify the uniqueness and inf-sup conditions of the Babuška-Brezzi theory to obtain wellposedness. To verify the uniqueness condition, we must prove that if

$$
\left(u, A_{h}^{*} v\right)_{K}+\langle q, v\rangle_{h}=0, \quad \forall v \in W_{h}^{*},
$$

then $u$ and $q$ vanishes. Since $q=D_{h} z$ for some $z \in V$, by virtue of Lemma 1.4.7, $\langle q, v\rangle_{h}=0$ for any $v$ in $V^{*}$. Hence (3.3.2) implies

$$
\left(u, A_{h}^{*} v\right)_{\Omega}=0, \quad \forall v \in V^{*}
$$

In particular, since $\mathcal{D}(\Omega)^{l} \subseteq V^{*}$, this implies that $A u=0$ and therefore $u \in W$. Hence 1.2 .3 and 1.4 .16 imply $\langle D u, v\rangle_{W^{*}}=0$, or equivalently $\left\langle D^{*} v, u\right\rangle_{W}=0$ for all $v \in V^{*}$. Thus $u \in{ }^{\perp} D^{*}\left(V^{*}\right)=V$. The bijectivity of $A: V \rightarrow L^{2}(\Omega)^{l}$ then implies 
that $u=0$. Returning to 3.3 .2 and setting $u=0$, we see that $\langle q, v\rangle_{h}=0$ for all $v \in W_{h}^{*}$, so $q=0$ as well.

It only remains to prove the inf-sup condition

$$
\|w\|_{W_{h}^{*}} \leq C_{1} \sup _{0 \neq x \in X} \frac{|b(x, w)|}{\|x\|_{X}}
$$

where $X=L^{2}(\Omega)^{m} \times Q$ and $b((u, q), w)=\left(u, A_{h}^{*} w\right)_{\Omega}+\langle q, w\rangle_{h}$. Given any $w \in$ $W_{h}^{*} \subseteq L^{2}(\Omega)^{l}$, we use the bijectivity of $A: V \rightarrow L^{2}(\Omega)^{l}$ and the Banach Open Mapping theorem to obtain a $v$ in $V$ satisfying $A v=w$, and $\|v\|_{W} \leq C\|w\|_{W_{h}^{*}}$. Then, setting $z=v+A_{h}^{*} w$ and $r=D_{h} v$, we have $\|r\|_{Q} \leq\|v\|_{W} \leq C\|w\|_{W_{h}^{*}}$ and $\|z\|_{\Omega} \leq(C+1)\|w\|_{W_{h}^{*}}$. Here and throughout this dissertation $C>0$ denotes an arbitrary constant. Hence

$$
\begin{aligned}
\|w\|_{W_{h}^{*}}^{2} & =\left(A_{h} v, w\right)_{\Omega}+\left(A_{h}^{*} w, A_{h}^{*} w\right)_{\Omega}=\left(v+A_{h}^{*} w, A_{h}^{*} w\right)_{\Omega}+\left\langle D_{h} v, w\right\rangle_{h} \\
& =\|(z, r)\|_{X} \frac{b((z, r), w)}{\|(z, r)\|_{X}} \leq C_{1}\|w\|_{W_{h}^{*}} \sup _{\substack{0 \neq x \in X\\
}} \frac{|b(x, w)|}{\|x\|_{X}},
\end{aligned}
$$

where $C_{1}$ depends only on $C$. Hence 1.4 .17 follows.

REMARK 1.4.8. Various elements of the arguments used in this proof are wellknown in the DPG literature - see e.g., [21, Section 6.2]. A generalization of these ideas to make a unified theory for DPG approximations of all Friedrichs systems was attempted in [8]. However, [8, equation (2.17)] is not correct (a counterexample is easily furnished by the Laplace example) and unfortunately that equation is used in [8, Lemma 2.4 and Corollary 2.5] to prove the existence of a solution for Problem 1.4.2. The above proof provides a corrigendum to [8] and shows that the results claimed there for symmetric Friedrichs systems are indeed correct for operators of the form (A-a) with $k=1$ and with $V$ and $V^{*}$ set respectively to the null spaces of the operators $B-M$ and $B+M^{*}$ defined there. 
REMARK 1.4.9. The above analysis is applicable beyond Friedrichs systems, as the example of Schrödinger equation in Chapter 4 will show. "Instead of working with one equation of higher than first order," writes Friedrichs in his early work [36], "we prefer to work with a system of equations of first order." The modern theory of Friedrichs systems (for operators of the form $\mathrm{A}-\mathrm{a}$ ) with $l=m$ ) starts with the assumption that $\left\|\left(A+A^{*}\right) \phi\right\|_{\Omega} \leq C\|\phi\|_{\Omega}$ for all $\phi \in \mathcal{D}(\Omega)^{l}-$ see [30, equation (T2)]. This assumption does not hold always, and that is the case for the Schrödinger operator.

1.4.2. An alternate proof of wellposedness. Another proof of Theorem 1.4 .5 can be obtained using the following two lemmas.

LEMMA 1.4.10. $V^{*}=\left\{y \in W_{h}^{*}:\langle q, y\rangle_{h}=0\right.$ for all $\left.q \in Q\right\}$.

Proof. If $y \in V^{*}$, then for any $z \in V$, using Lemmas 1.4.1 and 1.3.1, we have $\left\langle D_{h} z, y\right\rangle_{h}=\langle D z, y\rangle_{W^{*}}=0$, i.e., $\langle q, y\rangle_{h}=0$ for all $q \in Q$.

To prove the reverse containment, let $y \in W_{h}^{*}$ satisfy $\left\langle D_{h} z, y\right\rangle_{h}=0$ for all $z \in$ $V$. For any $\phi \in \mathcal{D}(\Omega)^{m}$, the distribution $A^{*} y$ satisfies $\left\langle A^{*} y, \phi\right\rangle_{\mathcal{D}(\Omega)^{m}}=(y, A \phi)_{\Omega}=$ $\left(A_{h}^{*} y, \phi\right)_{\Omega}+{\overline{\left\langle D_{h} \phi, y\right\rangle_{h}}}_{h}$ The last term is zero, because by $(\mathrm{A}-\mathrm{c}), \mathcal{D}(\Omega)^{m} \subseteq \operatorname{dom}(A)=$ $V$. Hence $A^{*} y=A_{h}^{*} y$ and $y$ is in $W^{*}$. Thus by Lemma 1.4.1, $\left\langle D_{h} z, y\right\rangle_{h}=\langle D z, y\rangle_{W}=$ 0 , so $y \in{ }^{\perp} D(V)$. Hence $y$ is in $V^{*}$ by Lemma 1.3.1.

Lemma 1.4.11. Suppose 1.4.9 holds. Then, for all $q \in Q$,

$$
\inf _{v \in D_{h, V}^{-1}(\{q\})}\|v\|_{W}=\sup _{0 \neq y \in W_{h}^{*}} \frac{\left|\langle q, y\rangle_{h}\right|}{\|y\|_{W_{h}^{*}}} .
$$


Proof. The supremum, denoted by $s$, is attained by the function $\tilde{u}_{q}$ in $W_{h}^{*}$ satisfying

$$
\begin{gathered}
\left(A_{h}^{*} \tilde{u}_{q}, A_{h}^{*} y\right)_{\Omega}+\left(\tilde{u}_{q}, y\right)_{\Omega}=-\langle q, y\rangle_{h}, \quad \forall y \in W_{h}^{*}, \text { and } \\
s=\left\|\tilde{u}_{q}\right\|_{W_{h}^{*}} .
\end{gathered}
$$

Choosing $y \in \mathcal{D}(\Omega)^{l}$ in 1.4 .18 , we conclude that the distribution $A\left(A_{h}^{*} \tilde{u}_{q}\right)$ is in $L^{2}(\Omega)^{l}$. Hence 1.4 .3 is applicable with $w=A_{h}^{*} \tilde{u}_{q}$ and we obtain

$$
\begin{aligned}
A_{h} A_{h}^{*} \tilde{u}_{q}+\tilde{u}_{q} & =0 \\
D_{h} A_{h}^{*} \tilde{u}_{q} & =q .
\end{aligned}
$$

Now let $u_{q}=A_{h}^{*} \tilde{u}_{q}$. Then 1.4.20a implies $A_{h} u_{q}=-\tilde{u}_{q}$, which implies $A_{h}^{*} A_{h} u_{q}=$ $-A_{h}^{*} \tilde{u}_{q}=-u_{q}$. Combining with $1.4 .20 \mathrm{~b}$, we have

$$
\begin{aligned}
A_{h}^{*} A_{h} u_{q}+u_{q} & =0 \\
D_{h} u_{q} & =q .
\end{aligned}
$$

Next, we show that $u_{q}$ is in $V$. By 1.4.9), it suffices to prove that $u_{q} \in{ }^{\perp} D^{*}\left(V^{*}\right)$. For any $\tilde{v}$ in $V^{*}$, we have, using Lemma 1.4.1. $\left\langle D^{*} \tilde{v}, u_{q}\right\rangle_{W}=-{\left.\overline{\left\langle D u_{q}, \tilde{v}\right.}\right\rangle_{W^{*}}}=-{\overline{\left\langle D_{h} u_{q}, \tilde{v}\right\rangle_{h}}}=$

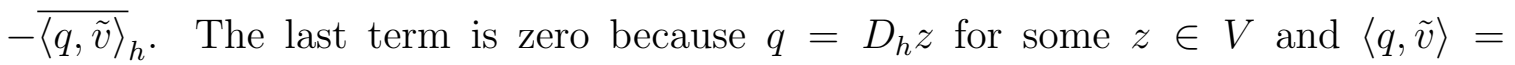
$\langle D z, \tilde{v}\rangle_{W^{*}}=0$ by Lemma 1.3.1. Hence $u_{q} \in{ }^{\perp} D^{*}\left(V^{*}\right)=V$.

The infimum of the lemma is $\|q\|_{Q}$. By virtue of $(1.4 .19)$, to complete the proof, it suffices to show that $\|q\|_{Q}=\left\|u_{q}\right\|_{W}=\left\|\tilde{u}_{q}\right\|_{W_{h}^{*}}$. The last equality is obvious from $u_{q}=A_{h}^{*} \tilde{u}_{q}$ and $A_{h} u_{q}=-\tilde{u}_{q}$. Hence we need only show that $\|q\|_{Q}=\left\|u_{q}\right\|_{W}$. Standard variational arguments show that the infimum defining $\|q\|_{Q}$ is attained by a unique minimizer $v_{q} \in V$ satisfying $\|q\|_{Q}=\left\|v_{q}\right\|_{W}, D_{h} v_{q}=q$ and $\left(A_{h} v_{q}, A_{h} v\right)_{\Omega}+\left(v_{q}, v\right)_{\Omega}=0$ for all $v \in D_{h, V}^{-1}(\{0\})$. Choosing a $v$ in $\mathcal{D}(K)$ (whose extension by zero is in $D_{h, V}^{-1}(\{0\})$ ), 
we conclude that distribution $\left.A^{*}\left(A_{h} v_{q}\right)\right|_{K}$ is in $L^{2}(K)^{m}$ for any $K \in \Omega_{h}$. Therefore $A_{h}^{*} A_{h} v_{q}$ is in $L^{2}(\Omega)^{m}$. In view of 1.4.21, this means that $v_{q}=u_{q}$.

Second proof of Theorem 1.4.5. According to [9, Theorem 3.3], it suffices to prove that there are positive constants $c_{0}, \hat{c}$ such that

$$
\begin{aligned}
c_{0}\|u\|_{\Omega} \leq \sup _{0 \neq y \in Y_{0}} \frac{\left|\left(u, A_{h}^{*} y\right)_{\Omega}\right|}{\|y\|_{W_{h}^{*}}} & \forall u \in L^{2}(\Omega)^{m}, \\
\hat{c}\|q\|_{Q} \leq \sup _{0 \neq y \in W_{h}^{*}} \frac{\left|\langle q, y\rangle_{h}\right|}{\|y\|_{W_{h}^{*}}} & \forall q \in Q .
\end{aligned}
$$

where $Y_{0}=\left\{y \in W_{h}^{*}:\langle q, y\rangle_{h}=0\right.$ for all $\left.q \in Q\right\}$.

Since 1.4.23) follows with $\hat{c}=1$ from Lemma 1.4.11, we only need to prove (1.4.22). First note that since $V$ is closed (by (1.4.9) $), A$ is a closed operator. By (1.4.10), the range of $A$ is closed. By the Closed Range Theorem for closed operators, range of $A^{*}$ is closed. Also, the well-known identity $\operatorname{ker}\left(A^{*}\right)=\operatorname{ran}(A)^{\perp}$, in combination with (1.4.10), implies that $A^{*}$ is injective. Hence there exists a $C>0$ such that

$$
C\|y\|_{\Omega} \leq\left\|A^{*} y\right\|_{\Omega} \quad \forall y \in \operatorname{dom}\left(A^{*}\right)=V^{*} .
$$

This implies the following inf-sup condition:

$$
C\|y\|_{W^{*}} \leq \sup _{u \in L^{2}(\Omega)^{m}} \frac{\left|\left(u, A^{*} y\right)_{\Omega}\right|}{\|u\|_{\Omega}} \quad \forall y \in V^{*} .
$$

To complete the proof, we note that by standard arguments the order of arguments in the inf and sup may be reversed to get

$$
\inf _{u \in L^{2}(\Omega)^{m}} \sup _{y \in V^{*}} \frac{\left|\left(u, A^{*} y\right)_{\Omega}\right|}{\|u\|_{\Omega}\|y\|_{W^{*}}}=\inf _{y \in V^{*}} \sup _{u \in L^{2}(\Omega)^{m}} \frac{\left|\left(u, A^{*} y\right)_{\Omega}\right|}{\|u\|_{\Omega}\|y\|_{W^{*}}} \geq C .
$$

By Lemma 1.4.10, $Y_{0}=V^{*}$, thus completing the proof of 1.4.22. 
REMARK 1.4.12. The idea behind Lemma 1.4.11 (to consider the two related problems (1.4.20) and 1.4.21), one with essential boundary conditions and the other with natural boundary conditions) was first presented in [9, 11], tailored to the specific needs of a Maxwell problem. A generalization for first order operators was presented later in 68. The argument to prove 1.4 .22 using the Closed Range Theorem, was first presented for the case of first order Sobolev spaces in [9, Theorem 6.6].

The last part of the theorem can be obtained by expressing $F$ in terms of $f$ as in the setting of Theorem 1.4.5. Then choosing $\phi \in \mathcal{D}(\Omega)^{l}$ we have

$$
\left(u, A_{h}^{*} \phi\right)_{\Omega}+\langle q, \phi\rangle_{h}=(f, \phi)_{\Omega}+\left\langle D_{h} g, \phi\right\rangle_{h}
$$

Thus, the distribution $A u$ is in $L^{2}(\Omega)^{l}$ and, by density that $A u=f \in L^{2}(\Omega)^{l}$, and $u \in W$. Returning to Problem 1.4 .2 we only need to prove that $u \in \in^{\perp} D^{*}\left(V^{*}\right)$.

We have, $0=-{\overline{\left\langle q, v^{*}\right\rangle_{h}}}=-{\overline{\left\langle D_{h}(u-g), v^{*}\right\rangle_{h}}}=-{\overline{\left\langle D(u-g), v^{*}\right\rangle_{W^{*}}}}_{\left\langle D^{*} v^{*}, u-g\right\rangle_{W}}$ for all $v^{*} \in V^{*}$. Thus $u \in V={ }^{\perp} D^{*}\left(V^{*}\right)$ and $q=D_{h} u$.

REMARK 1.4.13. A similar proof follows for the adjoint problem when $l=m$.

Next, we provide some results to prove the bijectivity condition that allow us to prove the bijectivity condition. Note that in order to prove that Assumption 1.4.10 holds in Theorem 1.4.5, we could alternatively show:

TheOREM 1.4.14. Suppose that 1.4.9) hold. If there is a constant $C>0$ such that $A: V \rightarrow L^{2}(\Omega)^{l}$ and $A^{*}: V^{*} \rightarrow L^{2}(\Omega)^{m}$ satisfies

$$
\begin{array}{cl}
\|A u\|_{\Omega} \geq C\|u\|_{W} & \forall u \in V, \text { and } \\
\left\|A^{*} v\right\|_{\Omega} \geq C\|v\|_{W} & \forall v \in V^{*},
\end{array}
$$

then 1.4.10 holds, i.e., $A: V \rightarrow L^{2}$ is a bijection. 
Proof. The inequality 1.4.26a and the closed range theorem for closed operators imply that $A: \operatorname{dom}(A)=V \rightarrow L^{2}(\Omega)^{l}$ is injective and has closed range. Moreover, its adjoint $A^{*}$ is injective (on its domain) by $1.4 .26 \mathrm{~b}$ ), so the range of $A$ must be all of $L^{2}(\Omega)^{l}$ (see e.g., [7, Corollary 2.18]). Hence $A$ is a bijection, i.e., condition 1.4.10 holds.

REMARK 1.4.15. Note that 1.4.26a gives a-priori error estimate for the strong problem, since $C\|u\|_{\Omega} \leq\|f\|_{\Omega}$.

\subsection{The ideal DPG method}

The ideal DPG method seeks $u_{h}$ and $q_{h}$ in finite-dimensional subspaces of $U_{h} \subset$ $L^{2}(\Omega)^{d+1}$ and $Q_{h} \subset Q$, satisfying

$$
b\left(\left(u_{h}, q_{h}\right), w_{h}\right)=F\left(w_{h}\right), \quad \text { for all } w_{h} \in T\left(U_{h} \times Q_{h}\right) .
$$

Here $T: L^{2}(\Omega) \times Q \rightarrow W_{h}$ is defined by $(T(z, r), v)_{h}=b((z, r), v)$ for all $v \in W_{h}$ and $(z, r) \in W_{h}$.

The main feature of the ideal DPG method is that the wellposedness of Problem 1.4 .2 implies quasioptimality of the method's error [19]. Moreover, it is well known [20] that there is a mixed method formulation that is equivalent to the above Petrov-Galerkin formulation (1.5.1). One of the variables in this mixed method is the error representation function $\varepsilon_{h} \in W_{h}^{*}$ defined by

$$
\left(\varepsilon_{h}, w\right)_{W_{h}^{*}}=(f, w)-b\left(\left(u_{h}, q_{h}\right), w\right), \quad \text { for all } w \in W_{h}^{*}
$$

One of the two equations in the mixed formulation given below is a restatement of this defining equation for $\varepsilon_{h} \in W_{h}^{*}$. The mixed formulation seeks $\varepsilon_{h} \in W_{h}^{*}$ and 
$\left(u_{h}, q_{h}\right) \in U_{h} \times Q_{h}$ such that

$$
\begin{array}{rr}
\left(\varepsilon_{h}, w\right)_{W_{h}^{*}}+b\left(\left(u_{h}, q_{h}\right), w\right)=F(w) & \text { for all } w \in W_{h}, \\
b\left((z, r), \varepsilon_{h}\right)=0 & \text { for all }(z, r) \in U_{h} \times Q_{h} .
\end{array}
$$

The main difficulty of the ideal method is that in order to compute $u_{h}$, one needs a basis for $W_{h}$, which must be obtained by applying $T$.

1.5.1. An Example of wellposed problem under this setting. For our model problem (1.1.3a), and following [18], we have $V=V^{*}=H(\operatorname{div}, \Omega) \times H_{0}^{1}(\Omega)$, and $A$ is given in by (1.1.4).

Lemma 1.5.1. Problem (1.4.2 is wellposed for this example, i.e, assumptions 1.4.9 and 1.4.10 of Theorem 1.4.5 hold.

Proof. Since $V=V^{*}$. It is easy to see, from the definition of $D$ in (1.2.5), that $V={ }^{\perp} D^{*}(V)$. Therefore, 1.4 .9$)$ is satisfied. Clearly, $A: V \rightarrow L^{2}(\Omega)$ satisfies the conditions 1.4.26 in Theorem 1.4.14, and therefore 1.4.10 is satisfied. Indeed, for any $u=\left(u_{\sigma}, u_{\mu}\right) \in V$ we have that $\left\|u_{\sigma}\right\| \leq\left\|u_{\sigma}-\nabla u_{\mu}\right\|+\left\|\nabla u_{\mu}\right\|$. The Poincaré inequality implies that $\left\|u_{\mu}\right\| \leq C\left\|\nabla u_{\mu}\right\|$ for all $u_{\mu} \in H_{0}^{1}(\Omega)$. Therefore $\|u\| \leq\|A u\|$ for any $u \in V$. The proof of $A^{*}: V \rightarrow L^{2}(\Omega)$ satisfying condition $1.4 .26 \mathrm{~b}$ is analogous.

REMARK 1.5.2. If we are able to prove trace theorems for the non-standard Hilbert spaces, we can characterize exactly the space $Q$. For example, in our model problem we have (1.4.6).

1.5.1.1. Numerical results for Example 1.1.1. In this section we present numerical results of the ideas described in this chapter for the broken weak formulation of the Laplace problem $(1.1 .2)$, by the ideal DPG method. 
For our model example, we consider $\Omega=(0,1)^{2}$, and $\left\{\Omega_{h}\right\}_{h \in I}$ a family of conforming shape regular simplicial finite element triangulations of $\Omega$. Define

$$
\begin{aligned}
& U_{h}=\left\{u \in L^{2}(\Omega)^{d+1}:\left.u\right|_{K} \in P_{p}(K)^{d+1} \text { for all } K \in \Omega_{h}\right\}, \\
& V_{h}=\left\{u \in H_{0}^{1}(\Omega)^{d+1} \cap C^{0}(\bar{\Omega})^{d+1}:\left.u\right|_{K} \in P_{p+1}(K)^{d+1} \text { for all } K \in \Omega_{h}\right\},
\end{aligned}
$$

where $P_{p}(K)$ is the space of polynomials of total degree $\leq p$ on $K$ and $C^{0}(D)$ denote the set of continuous functions on any domain $D$, as in [38]. It is well known that $Q_{h}=D_{h}\left(V_{h}\right)$ is given by

$$
Q_{h}=\left\{q \in Q: q_{\sigma_{n}} \in P_{p}(\partial K),\left.\quad q_{\mu}\right|_{\partial K} \in \tilde{P}_{p+1}(\partial K)\right\}
$$

where $\tilde{P}_{p+1}(\partial K)=P_{p}(\partial K) \cap C^{0}(\partial K)$ and $P_{p}(\partial K)=\left\{u:\left.u\right|_{F} \in P_{p}(F)\right.$ for all facets $F \in$ $\partial K\}$.

We now provide convergence rates for the ultraweak formulation when solving

$$
-\operatorname{div}(\operatorname{grad} \phi)=32 \pi^{2} \sin (\pi x) \sin (\pi y),
$$

on the unit square with homogeneous Dirichlet boundary conditions. The exact solution to this problem is given by

$$
\phi_{\text {exact }}(x, y)=16 \sin (\pi x) \sin (\pi y) .
$$

We considered a coarse triangular mesh of $\Omega$, and successive refinements of the mesh by connecting the mid points of the edges. In Figure 1.1 we observe that the order of convergence of this experiment is optimal, see [38]. The implementation has been done using the mixed form in 1.5 .2 and the finite element software NGSolve [60]. 


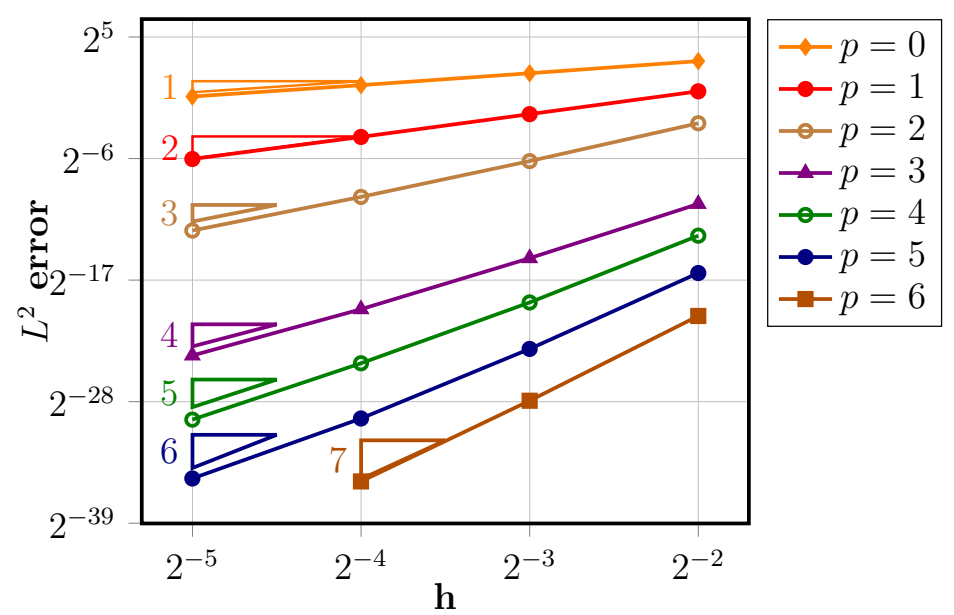

Figure 1.1. Rates of convergence for $u$ in the $L^{2}$-norm using a triangular mesh.

\subsection{Defining the $V$ and $V^{*}$ spaces from spaces of smooth functions}

Given a boundary value problem of the form (1.4.1), the first question that arises is: how to define the maximal space of functions where we can find a solution. We might think that, if the right hand side is smooth enough, then the solution will also need to be smooth. This brings the following ideas to the first attempt of finding the spaces $V$ and $V^{*}$.

Suppose that $V$ can be obtained by a density result from smooth functions satisfying the boundary conditions of the given problem, and that $A: V \rightarrow L^{2}(\Omega)^{l}$ is a bijection. Then we have an exact characterization of $V$, and many properties can be proved by density arguments.

Consider the boundary value problem $A u=f$, where $u \in L^{2}(\Omega)^{m}$ satisfies some boundary conditions in parts of the boundary of $\Omega$. To understand this in detail, let $\left\{\Gamma_{j}\right\}_{1 \leq j \leq m}$ and $\left\{\Gamma_{i}^{*}\right\}_{1 \leq i \leq l}$ be closed parts of the boundary of $\Omega$ where boundary conditions are imposed for the primal and adjoint problem respectively. We define

$$
\mathcal{V}=\left\{u \in \mathcal{D}(\bar{\Omega})^{m}:\left.(\gamma u)_{j}\right|_{\Gamma_{j}}=0, \quad 0 \leq j \leq m\right\}
$$


and

$$
\mathcal{V}^{*}=\left\{v \in \mathcal{D}(\bar{\Omega})^{l}:\left.\left(\gamma^{*} v\right)_{i}\right|_{\Gamma_{i}^{*}}=0, \quad 0 \leq i \leq l\right\}
$$

represent the spaces of smooth functions that satisfy the boundary conditions of the initial boundary value problem, and the boundary conditions of the adjoint problem respectively.

Here we have introduced two operators related to smooth functions. These operators are related with the operators of $D$ and $D^{*}$ when applied to smooth functions and acting as distributions. When $\gamma u=u$, then we are imposing Dirichlet boundary conditions on $\mathcal{V}$. However, other types of boundary conditions can be applied, as seen in Chapter 3 .

Therefore, we choose $\operatorname{dom}(A)$ to be defined by

$$
\operatorname{dom}(A)=\left\{u \in W:\langle D u, v\rangle_{W^{*}}=0, \forall v \in \mathcal{V}^{*}\right\}
$$

Since $\mathcal{D}(\Omega)^{m} \subset \operatorname{dom}(A)$, the adjoint is uniquely defined on

$$
\begin{aligned}
\operatorname{dom}\left(A^{*}\right)= & \left\{s \in L^{2}(\Omega)^{l}: \exists \ell \in L^{2}(\Omega)^{m}\right. \\
& \text { such that } \left.(A v, s)_{\Omega}=(v, \ell)_{\Omega}, \forall v \in \operatorname{dom}(A)\right\} .
\end{aligned}
$$

Finally, as in Section 1.3 , we set $V=\operatorname{dom}(A)$ and $V^{*}=\operatorname{dom}\left(A^{*}\right)$ with the understanding that both $V$ and $V^{*}$ are endowed with the $W$ and $W^{*}$-topology, respectively.

Observe that the choice of 1.6.1) implies that $V={ }^{\perp} D^{*}(\mathcal{V})$, since $\langle D u, v\rangle=$ $-\overline{\left\langle D^{*} v, u\right\rangle}$. Since we have verified the conditions of Lemma 1.3.1, we then have

$$
V^{*}={ }^{\perp} D(V) \text {. }
$$


Rewriting (2.1.5) in the same style,

$$
V={ }^{\perp} D^{*}\left(\mathcal{V}^{*}\right)
$$

Thus $V$ and $V^{*}$ are closed subspaces of $W$.

LEMMA 1.6.1. $\mathcal{V} \subset V$ and $\mathcal{V}^{*} \subset V^{*}$

Proof. Equation (2.1.2) implies $\left\langle D \phi, \phi^{*}\right\rangle_{W}=0$ for all $\phi \in V$ and $\phi^{*} \in \mathcal{V}^{*}$. Hence, any $\phi \in \mathcal{V}$ is also in ${ }^{\perp} D\left(\mathcal{V}^{*}\right)$, which by 4.2 .4 implies that $\phi \in V$. Thus $\mathcal{V} \subset V$.

If $\phi^{*} \in \mathcal{V}^{*}$, then 4.2 .2 shows that $\left\langle D \phi^{*}, u\right\rangle=0$ for all $u \in V$, i.e., $\phi^{*} \in{ }^{\perp} D(V)$, which by 4.2 .3 implies $\phi^{*}$ is in $V^{*}$.

In (4.2.4), $\mathcal{V}^{*}$ may be replaced by $V^{*}$ provided a density result is available, as we show next.

Assumption 1. Suppose $\mathcal{V}^{*}$ is dense in $V^{*}$ and $\mathcal{V}$ is dense in $V$.

Lemma 1.6.2. If Assumption 1 holds, then ${ }^{\perp} D\left(\mathcal{V}^{*}\right)={ }^{\perp} D\left(V^{*}\right)$ and ${ }^{\perp} D(\mathcal{V})=$ ${ }^{\perp} D(V)$.

Proof. Clearly $\mathcal{V}^{*} \subseteq V^{*}$ implies ${ }^{\perp} D\left(\mathcal{V}^{*}\right) \supseteq{ }^{\perp} D\left(V^{*}\right)$. To prove the reverse containment, suppose $w \in{ }^{\perp} D\left(\mathcal{V}^{*}\right)$ and $v^{*} \in V^{*}$. By density, there is a sequence $\tilde{v}_{n}$ in $\mathcal{V}^{*}$ satisfying $\lim _{n \rightarrow \infty}\left\|\tilde{v}_{n}-v^{*}\right\|_{W}=0$. Since $\left\langle D \tilde{v}_{n}, w\right\rangle_{W}=0$, by continuity of $D$, we have $\left\langle D v^{*}, w\right\rangle_{W}=0$. Hence $w \in{ }^{\perp} D\left(V^{*}\right)$. The second identity is proved similarly. 


\section{Hyperbolic systems in one space dimension}

The assumptions on which the previous theory is based can be verified for several examples. We begin with the simplest example in one space dimension in Section 2.1 where all the ideas are transparent. We then generalize to the example of multidimensional advection in Section 2.2 and establish a new trace theorem for the associated graph space. The final example in Section 2.4 considers a general symmetric hyperbolic system in one space dimension and leads into the discussion on the wave equation in the subsequent chapter. Contrary to Chapter 1, here we use $K$ to identify our domain instead of $\Omega$, since we will be solving local problem defined on elements $K$ of a spacetime mesh of $\Omega$ in the next chapter for the wave equation. The elements of the mesh of $\Omega$ will be generated such that they satisfy the causality condition. Note that all the ideas presented in Section 1.4 can be translated to one tent, by setting $\Omega_{h}$ equal to one element $K$. Mainly, the weak formulation of Problem 1.4.1 in one element $K$ is given by

Problem 2.0.1. (Weak formulation on $K$ ) Given any $F \in\left(W^{*}\right)^{\prime}$, find $u \in$ $L^{2}(K)^{m}$ and $q \in Q$ such that

$$
b((u, q), v)=F(v) \quad \forall v \in W^{*},
$$

where $F(v)=(f, v)-\langle D g, v\rangle \quad$ and $\quad b((u, q), v)=\left(u, A^{*} v\right)_{\Omega}+\langle q, v\rangle_{W^{*}}$. 


\subsection{The operator $\partial t$}

Let $K$ be an open triangle in spacetime $(x, t) \in \mathbb{R} \times \mathbb{R}$, with vertices at $(x, t)=$ $(0,0),(1,0)$, and $(1,1)$ as in Figure 2.1. We split the boundary of $K$ into an inflow, outflow, and a characteristic part:

$$
\begin{gathered}
\partial_{\mathrm{i}} K=\{(x, t) \in \partial K: t=0\}, \quad \partial_{\mathrm{o}} K=\{(x, t) \in \partial K: x=t\}, \\
\partial_{\mathrm{c}} K=\{(x, t) \in \partial K: x=1\} .
\end{gathered}
$$

(See Figure 2.1). We are interested in the boundary value problem of finding $u$ such that

$$
\left\{\begin{array}{c}
\partial_{t} u=f \quad \text { in } K, \\
\left.u\right|_{\partial_{\mathrm{i}} K}=0 .
\end{array}\right.
$$

Obviously, the operator $A=\partial_{t}$ and the formal adjoint defined by $A^{*}=-\partial_{t}$ satisfy

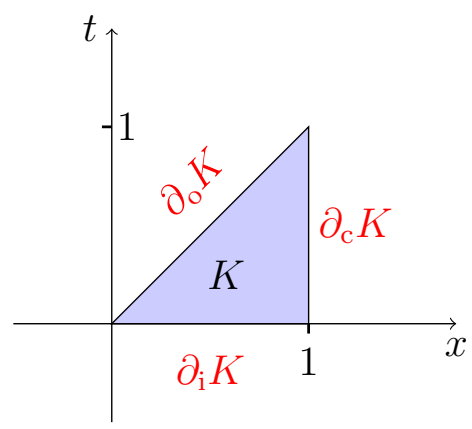

Figure 2.1. Reference domain $K \subset \mathbb{R}^{2}$

the conditions (A-a) and A-b when setting $\Omega=K, d=2$, and $m=l=k=1$ in Chapter 1. The graph space is given by $W=\left\{u \in L^{2}(K): \partial_{t} u \in L^{2}(K)\right\}$ and $W^{*}=W$. Here $\langle\cdot, \cdot\rangle_{W}$ represents the duality pairing in $W$. We define the operator $D$ such that $D: W \rightarrow W^{\prime}$ and it satisfies: $\langle D u, v\rangle_{W}=(A u, v)-\left(u, A^{*} v\right)$. Here $\partial_{t}$ is taken in the distributional sense. Note that $\mathcal{D}(\bar{K}) \subset W$. Hence, the boundary 
operator $D$ on smooth functions $u, v \in \mathcal{D}(\bar{K})$ is given by

$$
\begin{aligned}
\langle D u, v\rangle_{W} & =\int_{0}^{1} \int_{0}^{x}\left(\partial_{t} u\right) v d x d t+\int_{0}^{1} \int_{0}^{x} u\left(\partial_{t} v\right) d x d t \\
& =\int_{0}^{1} u(x, x) v(x, x)-u(x, 0) v(x, 0) d x
\end{aligned}
$$

In order to define the domain of $A$ and $A^{*}$, we introduce the following subspaces of $\mathcal{D}(\bar{K})$ :

$$
\mathcal{V}=\{u \in \mathcal{D}(\bar{K}): u(x, 0)=0\}
$$

and

$$
\mathcal{V}^{*}=\{v \in \mathcal{D}(\bar{K}): v(x, x)=0\}
$$

We set the $\operatorname{dom}(A)$ to be defined as

$$
\begin{aligned}
\operatorname{dom}(A) & =\left\{u \in W:\langle D u, v\rangle_{W}=0 \forall v \in \mathcal{V}^{*}\right\} \\
& =\left\{u \in W:\langle D v, u\rangle_{W}=0, \forall v \in \mathcal{V}^{*}\right\}
\end{aligned}
$$

Thus, it is easy to see that Assumption A-c is satisfied. Note that $\mathcal{D}(K) \subset \operatorname{dom}(A)$, thus the operator $A$ is densely defined, and therefore the domain of the adjoint is unique and given by

$$
\begin{aligned}
\operatorname{dom}\left(A^{*}\right)= & \left\{s \in L^{2}(\Omega): \text { there is an } \ell \in L^{2}(\Omega)\right. \\
& \text { such that } \left.\left(\partial_{t} v, s\right)_{\Omega}=(v, \ell)_{\Omega} \text { for all } v \in \operatorname{dom}(A)\right\} .
\end{aligned}
$$

Finally, as in Chapter 1, we set $V=\operatorname{dom}(A)$ and $V^{*}=\operatorname{dom}\left(A^{*}\right)$ with the understanding that both $V$ and $V^{*}$ are endowed with the $W$-topology, while $\operatorname{dom}(A)$ and $\operatorname{dom}\left(A^{*}\right)$ have the topology of $L^{2}(\Omega)$. 
Since we have verified the assumptions of Lemma 1.3.1, we then have

$$
V^{*}={ }^{\perp} D(V) \text {. }
$$

Rewriting (2.1.5) in the same style,

$$
V={ }^{\perp} D\left(\mathcal{V}^{*}\right)
$$

Thus $V$ and $V^{*}$ are closed subspaces of $W$.

$$
\text { LEMMA 2.1.1. } \mathcal{V} \subset V \text { and } \mathcal{V}^{*} \subset V^{*}
$$

Proof. If $v \in \mathcal{V}$, then the definition of the boundary operator applied to smooth functions in 2.1.2 and $\mathcal{V}^{*}$ in 2.1.4 imply that $\left\langle D v, v^{*}\right\rangle_{W}=0$ for all $v^{*} \in \mathcal{V}^{*}$, thus $v \in V$. In the same way, if $v^{*} \in \mathcal{V}^{*}$, then following the definition of $\operatorname{dom}(A)$ we have $\left\langle D u, v^{*}\right\rangle=0$ for all $u \in \operatorname{dom}(A)$, where $\left\langle D u, v^{*}\right\rangle_{W}=\left(\partial_{t} u, v^{*}\right)+\left(u, \partial_{t} v^{*}\right)$. Thus, $v \in \operatorname{dom}\left(A^{*}\right)$, since we had found $\ell=-\partial_{t} v^{*} \in L^{2}(K)$ in 2.1 .6$)$.

In $(2.1 .8), \mathcal{V}^{*}$ may be replaced by $V^{*}$ provided a density result is available, as we show next.

Lemma 2.1.2. If Assumption 1 holds in this example, when operator $A=\partial_{t}$, then ${ }^{\perp} D\left(\mathcal{V}^{*}\right)={ }^{\perp} D\left(V^{*}\right)$ and ${ }^{\perp} D(\mathcal{V})={ }^{\perp} D(V)$.

Proof. This is an immediate conclusion from Lemma 1.6.2.

2.1.1. Density result. In order to prove Assumption 1, we will provide a trace lemma for the $W$ space.

Because $\operatorname{dist}\left(\partial_{\mathrm{i}} K, \partial_{\mathrm{o}} K\right)=0$, although the operator $D$ is defined on all $W$, we must be careful in speaking of traces of functions in $W$ on these boundary parts. Indeed, $w(x, t)=x^{-1 / 2}$ is in $W$, since $\|w\|_{K}^{2}=\int_{0}^{1} \int_{0}^{x} \frac{1}{x} d t d x=1$, and $\left\|\partial_{t} w\right\|_{K}=0$, but its restriction to $\partial_{\mathrm{i}} K$ is not in $L^{2}\left(\partial_{\mathrm{i}} K\right)$. 
To study this further, we define the maps

$$
\tau_{\mathrm{i}}: v(x, t) \mapsto v(x, 0) \quad \text { and } \quad \tau_{\mathrm{o}}: v(x, t) \mapsto v(x, x)
$$

whose application to any function gives its traces on $\partial_{\mathrm{i}} K$ and $\partial_{\mathrm{o}} K$, respectively. These maps are obviously well-defined for smooth functions. Below we prove that they extend to $W$. Let $L_{\delta}^{2}(S)$ denote the set of all measurable functions $s$ on $S$ with finite $\int_{S} \delta s^{2}$, with $\delta$ a strictly positive function.

Lemma 2.1.3. For the space $W$ in this example, the following maps are continuous:

$$
\tau_{\mathrm{i}}: W \rightarrow L_{x}^{2}(0,1), \quad \tau_{\mathrm{o}}: W \rightarrow L_{x}^{2}(0,1), \text { and } \tau_{\mathrm{i}}-\tau_{\mathrm{o}}: W \rightarrow L_{1 / x}^{2}(0,1)
$$

i.e., there is a constant $C_{0}>0$ such that

$$
\int_{0}^{1} x\left|\tau_{\mathrm{i}} w\right|^{2} d x+\int_{0}^{1} x\left|\tau_{\mathrm{o}} w\right|^{2} d x+\int_{0}^{1} \frac{\left|\tau_{\mathrm{i}} w-\tau_{\mathrm{o}} w\right|^{2}}{x} d x \leq C_{0}\|w\|_{W}^{2}
$$

for all $w \in W$.

Proof. A general density result for first order systems in [42, Theorem 4] implies that $\mathcal{D}(\bar{K})$ is dense in $W$, so it suffices to prove 2.1 .9 for all $w \in \mathcal{D}(\bar{K})$. Beginning with the fundamental theorem of calculus,

$$
\tau_{\mathrm{i}} w(x)=w(x, r)-\int_{0}^{r} \partial_{t} w(x, t) d t
$$

since $0 \leq x \leq 1$, squaring, integrating over $r$, and overestimating, we have

$$
x\left|\tau_{\mathrm{i}} w(x)\right|^{2}=\int_{0}^{x}\left|\tau_{\mathrm{i}} w(x)\right|^{2} d r \leq 2 \int_{0}^{x}|w(x, r)|^{2} d r+2 \int_{0}^{x} r \int_{0}^{r}\left|\partial_{t} w(x, t)\right|^{2} d t d r .
$$


Now integrating over $x$ and overestimating again,

$$
\begin{aligned}
\frac{1}{2} \int_{0}^{1} x\left|\tau_{\mathrm{i}} w(x)\right|^{2} d x & \leq \int_{0}^{1} \int_{0}^{x}|w(x, r)|^{2} d r d x+\int_{0}^{1} \int_{0}^{1} 1 \int_{0}^{x}\left|\partial_{t} w(x, t)\right|^{2} d t d r d x \\
& =\|w\|_{W}^{2} .
\end{aligned}
$$

A similar argument shows that the same inequality holds with $\tau_{\mathrm{i}}$ replaced by $\tau_{\mathrm{o}}$, using the identity

$$
\tau_{\mathrm{o}} w(x)=w(x, r)+\int_{r}^{x} \partial_{t} w(x, t) d t
$$

Since $0 \leq x \leq 1$ we have

$$
\begin{aligned}
x\left|\tau_{\mathrm{o}} w(x)\right|^{2} & =\int_{0}^{x}\left|\tau_{\mathrm{o}} w(x)\right|^{2} d r \leq 2 \int_{0}^{x}|w(x, r)|^{2} d r+2 \int_{0}^{x}\left(\int_{r}^{x} \partial_{t} w(x, t) d t\right)^{2} d r \\
& \leq 2 \int_{0}^{x}|w(x, r)|^{2} d r+2 \int_{0}^{x}\left(\int_{r}^{x} 1 d r\right)\left(\int_{r}^{x}\left|\partial_{t} w(x, t)\right|^{2} d t\right) d r \\
& \leq 2 \int_{0}^{x}|w(x, r)|^{2} d r+2 \int_{0}^{x}(x-r)\left(\int_{r}^{x}\left|\partial_{t} w(x, t)\right|^{2} d t\right) d r .
\end{aligned}
$$

Thus,

$$
\frac{1}{2} \int_{0}^{1} x\left|\tau_{\mathrm{o}} w(x)\right|^{2} d x \leq\|w\|_{W}^{2}
$$

Finally, we prove that the inequality

$$
\int_{0}^{1} \frac{\left|\tau_{\mathrm{i}} w-\tau_{\mathrm{o}} w\right|^{2}}{x} d x \leq\|w\|_{W}^{2}
$$

holds. This statement is true since

$$
\left|\tau_{\mathrm{i}} w(x)-\tau_{\mathrm{o}} w(x)\right|^{2}=\left|\int_{0}^{x} \partial_{t} w(x, s) d s\right|^{2} \leq x \int_{0}^{x}\left|\partial_{t} w(x, s)\right|^{2} d s .
$$

Thus, dividing through by $x$ and integrating over $x$ we obtain the result. 
Lemma 2.1.4. The following two identities are well defined for any $u, v \in W$.

$$
\langle D u, v\rangle_{W}=\int_{0}^{1}\left(\frac{\tau_{\mathrm{o}} u-\tau_{\mathrm{i}} u}{x^{1 / 2}}\right)\left(x^{1 / 2} \tau_{\mathrm{o}} v\right) d x+\int_{0}^{1}\left(\frac{\tau_{\mathrm{o}} v-\tau_{\mathrm{i}} v}{x^{1 / 2}}\right)\left(x^{1 / 2} \tau_{\mathrm{i}} u\right) d x
$$

and

$$
\langle D u, v\rangle_{W}=\int_{0}^{1}\left(\frac{\tau_{\mathrm{o}} v-\tau_{\mathrm{i}} v}{x^{1 / 2}}\right)\left(x^{1 / 2} \tau_{\mathrm{o}} u\right) d x+\int_{0}^{1}\left(\frac{\tau_{\mathrm{o}} u-\tau_{\mathrm{i}} u}{x^{1 / 2}}\right)\left(x^{1 / 2} \tau_{\mathrm{i}} v\right) d x
$$

ProOF. We will use the density result for first order systems as before [42, Theorem 4]. Let $u, v$ in $\mathcal{D}(\bar{K})$. Note that expression 2.1.11a can be obtained by adding and subtracting $\left(\tau_{\mathrm{o}} v\right)\left(\tau_{\mathrm{i}} u\right)$ in the second equality of 2.1.2). Thus, for any $u, v \in C^{1}(\bar{K})$ we have

$$
\begin{aligned}
\langle D u, v\rangle_{W} & =\int_{0}^{1}\left(\tau_{\mathrm{o}} v\right)\left(\tau_{\mathrm{o}} u\right)-\left(\tau_{\mathrm{o}} v\right)\left(\tau_{\mathrm{i}} u\right)+\left(\tau_{\mathrm{o}} v\right)\left(\tau_{\mathrm{i}} u\right)-\left(\tau_{\mathrm{i}} v\right)\left(\tau_{\mathrm{i}} u\right) d x . \\
& =\int_{0}^{1}\left(\frac{\tau_{\mathrm{o}} u-\tau_{\mathrm{i}} u}{x^{1 / 2}}\right)\left(x^{1 / 2} \tau_{\mathrm{o}} v\right) d x+\int_{0}^{1}\left(\frac{\tau_{\mathrm{o}} v-\tau_{\mathrm{i}} v}{x^{1 / 2}}\right)\left(x^{1 / 2} \tau_{\mathrm{i}} u\right) d x .
\end{aligned}
$$

In the same way, by adding and subtracting $\left(\tau_{\mathrm{o}} u\right)\left(\tau_{\mathrm{i}} v\right)$ in the second equality of 2.1.2), we obtain $(2.1 .11 b)$.

Now, using the Cauchy-Schwarz inequality, the continuity property of the traces proved in Lemma 2.1.3 and the density of $C^{1}(\bar{K})$ in $W$, we can extend these integrals continuously to $W$. Hence 2.1.11a and 2.1.11b holds for all $v$ and $w$ in $W$.

Lemma 2.1.5. (Characterization of the spaces $V$ and $V^{*}$ using the trace lemma). For this example, and following the setting of Chapter 1 , the spaces $V$ and $V^{*}$ can 
also be represented by the following sets

$$
V=\left\{u \in W: \tau_{\mathrm{i}}(u)=0\right\} \quad \text { and } \quad V^{*}=\left\{v \in W: \tau_{\mathrm{o}}(v)=0\right\}
$$

Proof. First we prove $V \subseteq\left\{u \in W: \tau_{i}(u)=0\right\}$. If $u \in \operatorname{dom}(A)$, then 2.1.5 and 2.1.11a imply

$$
0=\langle D u, v\rangle_{W}=\int_{0}^{1}\left(\frac{0-\tau_{\mathrm{i}} v}{x^{1 / 2}}\right)\left(x^{1 / 2} \tau_{\mathrm{i}} u\right)=\int_{0}^{1}\left(-\tau_{\mathrm{i}} v\right)\left(\tau_{\mathrm{i}} u\right) d x \text { for all } \in \mathcal{V}^{*}
$$

Since $\tau_{i} v \in \mathcal{D}(0,1)$ for any $v \in \mathcal{V}^{*}$, and by the density of $\mathcal{D}(0,1)$ in $L^{2}(0,1)$, we obtain $\tau_{\mathrm{i}} u=0$ a.e. in $(0,1)$. Thus, $u \in\left\{w \in W: \tau_{\mathrm{i}}(w)=0\right\}$. For the reverse inclusion let $u \in\left\{w \in W: \tau_{\mathrm{i}}(w)=0\right\}$. Using again 2.1.11a we conclude that $\langle D \phi, u\rangle=0$ for all $\phi \in \mathcal{V}^{*}$, thus $u \in \operatorname{dom}(A)$.

Analogously, using 2.1.11b we obtain $V^{*}=\left\{v \in W: \tau_{\mathrm{o}}(v)=0\right\}$.

Lemma 2.1.6. Assumption 1 holds for this example, i.e. $\mathcal{V}^{*}$ is dense in $V^{*}$, and $\mathcal{V}$ is a dense subset of $V$.

Proof. Given any $v \in V^{*}$, by the density of $C^{1}(\bar{K})$ in $W$, there is a sequence $w_{n} \in C^{1}(\bar{K})$ converging to $v$ in $W$. Let $v_{n}(x, t)=w_{n}(x, t)-\left(\tau_{\mathrm{o}} w_{n}\right)(x)$. Since $\tau_{\mathrm{o}}\left(v_{n}\right)=0$, we conclude that $v_{n} \in \mathcal{V}^{*}$, for all $n$ (definition (2.1.4)). Since we also know $\partial_{t}\left(\tau_{\mathrm{o}} w_{n}(x)\right)=0$, we obtain

$$
\begin{aligned}
\left\|v_{n}-v\right\|_{W} & \leq\left\|w_{n}-v\right\|_{W}+\left\|\tau_{\mathrm{o}} w_{n}\right\|_{L^{2}(K)} \\
& =\left\|w_{n}-v\right\|_{W}+\left(\int_{0}^{1} \int_{0}^{x}\left|w_{n}(x, x)\right|^{2} d t d x\right)^{1 / 2} \\
& =\left\|w_{n}-v\right\|_{W}+\left\|\tau_{\mathrm{o}} w_{n}\right\|_{L_{x}^{2}(0,1)} \\
& =\left\|w_{n}-v\right\|_{W}+\left\|\tau_{\mathrm{o}}\left(w_{n}-v\right)\right\|_{L_{x}^{2}(0,1)} .
\end{aligned}
$$


This, together with Lemma 2.1.3, and the convergence of $w_{n}$ to $v$ in $W$, imply the convergence of $v_{n}$ to $v$ in $W$. Thus $\mathcal{V}^{*}$ is dense in $V^{*}$. Similarly, $\mathcal{V}$ is dense in $V$.

LEMma 2.1.7. The conditions of Theorem 1.4.14 are satisfied for this example.

Proof. Following the result of Lemma 2.1.6, it suffices to prove the conditions of Theorem of $(1.4 .26)$ for the dense subsets. For any function $v$ in $\mathcal{V}$, we have

$$
v(x, t)^{2}=\int_{0}^{t} \frac{\partial}{\partial s}\left(v(x, s)^{2}\right) d s=\int_{0}^{t} 2 v(x, s) \frac{\partial}{\partial s} v(x, s) d s,
$$

which implies

$$
\begin{aligned}
\int_{0}^{1} \int_{0}^{x} v(x, t)^{2} d t d x & \leq 2 \int_{0}^{1} \int_{0}^{x}\left(\int_{0}^{t} v(x, s)^{2} d s\right)^{1 / 2}\left(\int_{0}^{t}\left|\frac{\partial}{\partial s} v(x, s)\right|^{2} d s\right)^{1 / 2} d t d x \\
& \leq 2 \int_{0}^{1}\left(\int_{0}^{x} \int_{0}^{t} v(x, s)^{2} d s d t\right)^{1 / 2}\left(\int_{0}^{x} \int_{0}^{t}\left|\frac{\partial}{\partial s} v(x, s)\right|^{2} d s d t\right)^{1 / 2} d x
\end{aligned}
$$

Since $x \leq 1$, this shows that $\|v\|_{\Omega} \leq 2\|A v\|_{\Omega}$ for all $v \in \mathcal{V}$ and hence for all $v \in V$. The proof of $1.4 .26 \mathrm{~b}$ is analogous using the identity

$$
v^{*}(x, t)^{2}=\int_{x}^{t} \frac{\partial}{\partial s}\left(v^{*}(x, s)^{2}\right) d s=\int_{x}^{t} 2 v^{*}(x, s) \frac{\partial}{\partial s} v^{*}(x, s) d s, \quad \text { for all } v^{*} \in \mathcal{V}^{*}
$$

THEOREM 2.1.8. Problem 1.4.2 is well posed for this example, when $\Omega_{h}$ consists of only one element, namely $K$.

Proof. By Lemmas 2.1.6 and 2.1.7, assumptions 1.4.9 and 1.4.26 hold. Therefore Theorem 1.4.14 implies that 1.4 .10 holds. Thus, Theorem 1.4 .5 gives the required result. 


\subsection{Unidirectional advection}

The above calculations have a straightforward generalization to multidimensional tent-shaped domains. We say that $K_{0}$ is a vertex patch around a point $p$ if it is an open polyhedron in $\mathbb{R}^{d-1}(d>1)$ that can be partitioned into a finite number of $(d-1)$-simplices with a common vertex $p \in \mathbb{R}^{d-1}$.

We first consider domains $K$ built on (spatial) vertex patches of the form

$$
K=\left\{(x, t): x \in K_{0}, g_{\mathrm{i}}(x)<t<g_{\mathrm{o}}(x)\right\}
$$

(and later, after Definition 2.3.1 below, specialize to tent-shaped domains). Above, $g_{\mathrm{o}}(x)$ and $g_{\mathrm{i}}(x)$ are Lipschitz functions on $K_{0}$ such that $K$ is a nonempty open set in $\mathbb{R}^{d}$. Then the unit outward normal vector $n=\left(n_{x}, n_{t}\right)$ exists a.e. on $\partial K$. Continuing to consider the same operator as in Section 2.1, namely $A=\partial_{t}$, but on the new domain $K$, the following defines inflow, outflow, and characteristic parts of the boundary:

$$
\begin{gathered}
\partial_{\mathrm{i}} K=\left\{(x, t) \in \partial K: n_{t}<0\right\}, \quad \partial_{\mathrm{o}} K=\left\{(x, t) \in \partial K: n_{t}>0\right\}, \\
\partial_{\mathrm{c}} K=\left\{(x, t) \in \partial K: n_{t}=0\right\} .
\end{gathered}
$$

We define the space $W$ as the graph space, and notice that $W=W^{*}$, and thus the operator $D$ is defined as previously such that $D: W \rightarrow W^{\prime}$ and it satisfies

$$
\langle D u, v\rangle_{W}=(A u, v)_{K}-\left(u, A^{*} v\right)_{K}
$$

Note that Assumptions $\mathrm{A}-\mathrm{a}$ and $\mathrm{A}-\mathrm{b}$ hold, when setting $l=m=1$ in Chapter 1. The boundary operator for the unidirectional advection operator is given by

$$
\langle D v, u\rangle=\int_{K_{0}} \int_{g_{\mathrm{i}}(x)}^{g_{\mathrm{o}}(x)} \partial_{t} u \cdot v+u \cdot \partial_{t} v d t d x \quad \text { for all } u, v \in W
$$


which for smooth functions $u, v \in \mathcal{D}(\bar{K})$, is expressed by

$$
\langle D v, u\rangle=\int_{K_{0}} u\left(x, g_{\mathrm{o}}(x)\right) \cdot v\left(x, g_{\mathrm{o}}(x)\right)-u\left(x, g_{\mathrm{i}}(x)\right) \cdot v\left(x, g_{\mathrm{i}}(x)\right) d x .
$$

We define, as in the previous section,

$$
\begin{gathered}
\mathcal{V}=\left\{u \in \mathcal{D}(\bar{K}): u\left(x, g_{\mathrm{i}}(x)\right)=0\right\}, \\
\mathcal{V}^{*}=\left\{v \in \mathcal{D}(\bar{K}): v\left(x, g_{\mathrm{o}}(x)\right)=0\right\},
\end{gathered}
$$

and

$$
\operatorname{dom}(A)=\left\{u \in W:\langle D v, u\rangle_{W}=0, \forall v \in \mathcal{V}^{*}\right\}
$$

Note that $\mathcal{D}(K) \subset \operatorname{dom}(A)$, by 2.2.3 and definition 2.2.5. Thus Assumption (A-c) is satisfied. Therefore, $A$ is a densely defined operator and the domain of the adjoint is given by

$$
\begin{aligned}
\operatorname{dom}\left(A^{*}\right)= & \left\{s \in L^{2}(\Omega): \exists \ell \in L^{2}(\Omega)\right. \\
& \text { such that } \left.\left(\partial_{t} v, s\right)_{\Omega}=(v, \ell)_{\Omega} \quad \forall v \in \operatorname{dom}(A)\right\} .
\end{aligned}
$$

We now denote $\operatorname{dom}(A)$ by $V$ and $\operatorname{dom}\left(A^{*}\right)$ by $V^{*}$, when these spaces are endowed with the topology of $W$. 
Theorem 2.2.1. Let $K$ be as in 2.2.1) and let $A=\partial_{t}$, the spaces $\mathcal{V}^{*}$ and $\mathcal{V}$ defined by (2.2.7) and 2.2.5), and $V$ and $V^{*}$ given by (2.2.6) and 2.2.7) respectively.

Then

(i) The inflow and outflow trace maps,

$$
\tau_{\mathrm{i}}: v(x, t) \mapsto v\left(x, g_{\mathrm{i}}(x)\right) \text { and } \tau_{\mathrm{o}}: v(x, t) \mapsto v\left(x, g_{\mathrm{o}}(x)\right)
$$

extend to continuous linear operators

$$
\tau_{\mathrm{i}}: W \rightarrow L_{g_{\mathrm{o}}-g_{\mathrm{i}}}^{2}\left(K_{0}\right), \quad \tau_{\mathrm{o}}: W \rightarrow L_{g_{\mathrm{o}}-g_{\mathrm{i}}}^{2}\left(K_{0}\right), \quad \text { and } \quad \tau_{\mathrm{i}}-\tau_{\mathrm{o}}: W \rightarrow L_{1 /\left(g_{\mathrm{o}}-g_{\mathrm{i}}\right)}^{2}\left(K_{0}\right)
$$

(ii) (Characterization of the spaces $V$ and $V^{*}$ using the trace theorem of part (i)). The spaces $V$ and $V^{*}$ satisfy

$$
V=\left\{u \in W: \tau_{\mathrm{i}} u=0\right\} \quad \text { and } \quad V^{*}=\left\{u \in W: \tau_{\mathrm{o}} u=0\right\}
$$

(iii) $\mathcal{V}$ is dense in $V$ and $\mathcal{V}^{*}$ is dense in $V^{*}$.

Therefore, conditions of Theorem 1.4 .10 holds and Problem 1.4.2 is wellposed on $K$.

Proof. The proof is an extension of the previous section. To prove (i), we use the fundamental fact that $\mathcal{D}(K)$ is dense in $W$ for $K \subset \mathbb{R}^{d}$ (see [42, Theorem 4]). To prove the first continuity result, choose $w \in \mathcal{D}(K)$ and use the identity $\tau_{\mathrm{i}} w(x)=$ $w(x, r)-\int_{g_{\mathrm{i}}(x)}^{r} \partial_{t} w(x, t) d t$, for any $r$ such that $g_{\mathrm{i}}(x)<r \leq g_{\mathrm{o}}(x)$. Therefore,

$$
\begin{aligned}
\left(g_{\mathrm{o}}(x)-g_{\mathrm{i}}(x)\right)\left|\tau_{\mathrm{i}} w(x)\right|^{2} & =\int_{g_{\mathrm{i}}(x)}^{g_{\mathrm{o}}(x)}\left|\tau_{\mathrm{i}} w(x)\right|^{2} d r \\
& \leq 2\left(\int_{g_{\mathrm{i}}(x)}^{g_{\mathrm{o}}(x)}|w(x, r)|^{2} d r+\int_{g_{\mathrm{i}}(x)}^{g_{\mathrm{o}}(x)}\left(r-g_{i}(x)\right) \int_{g_{\mathrm{o}}(x)}^{r}\left|\partial_{t} w(x, t)\right|^{2} d t d r\right) .
\end{aligned}
$$


Now, integrating over $K_{0}$, we have

$$
\begin{aligned}
\int_{K_{0}}\left(g_{\mathrm{o}}(x)-g_{\mathrm{i}}(x)\right)\left|\tau_{\mathrm{i}} w(x)\right|^{2}= & \int_{K_{0}} \int_{g_{\mathrm{i}}(x)}^{g_{\mathrm{o}}(x)}\left|\tau_{\mathrm{i}} w(x)\right|^{2} d r \\
\leq & 2 \int_{K_{0}} \int_{g_{\mathrm{i}}(x)}^{g_{\mathrm{o}}(x)}|w(x, r)|^{2} d r \\
& +2 \int_{K_{0}} \int_{g_{\mathrm{i}}(x)}^{g_{\mathrm{o}}(x)}\left(r-g_{i}(x)\right) \int_{g_{\mathrm{o}}(x)}^{r}\left|\partial_{t} w(x, t)\right|^{2} d t d r .
\end{aligned}
$$

Then, $\int_{K_{0}}\left(g_{\mathrm{o}}(x)-g_{\mathrm{i}}(x)\right)\left|\tau_{\mathrm{i}} w(x)\right|^{2} \leq 2 \max _{x \in K_{0}}\left\{1, g_{\mathrm{o}}(x)-g_{\mathrm{i}}(x)\right\}\|w\|_{W}^{2}$ and we extend the result to $W$ due to the density result.

Analogously we use the identity $\tau_{\mathrm{o}} w(x)=w(x, r)+\int_{r}^{g_{\mathrm{o}}(x)} \partial_{t} w(x, t) d t$, to obtain

$$
\begin{aligned}
\left(g_{\mathrm{o}}-g_{\mathrm{i}}\right)(x)\left|\tau_{\mathrm{o}} w(x)\right|^{2} & =\int_{g_{\mathrm{i}}(x)}^{g_{\mathrm{o}}(x)}\left|\tau_{\mathrm{o}} w(x)\right|^{2} d r \\
& \leq 2 \int_{g_{\mathrm{i}}(x)}^{g_{\mathrm{o}}(x)}|w(x, r)|^{2} d r+2 \int_{0}^{x}\left(\int_{r}^{x} \partial_{t} w(x, t) d t\right)^{2} d r \\
& \leq 2\left(\int_{g_{\mathrm{i}}(x)}^{g_{\mathrm{o}}(x)}|w(x, r)|^{2} d r+\int_{g_{\mathrm{i}}(x)}^{g_{\mathrm{o}}(x)}\left(\int_{r}^{g_{\mathrm{o}}(x)} 1 d t\right)\left(\int_{r}^{x}\left|\partial_{t} w(x, t)\right|^{2} d t\right) d r\right) \\
& \leq 2\left(\int_{g_{\mathrm{i}}(x)}^{g_{\mathrm{o}}(x)}|w(x, r)|^{2} d r+\int_{g_{\mathrm{i}}(x)}^{g_{\mathrm{o}}(x)}\left(g_{\mathrm{o}}(x)-r\right)\left(\int_{r}^{x}\left|\partial_{t} w(x, t)\right|^{2} d t\right) d r\right) .
\end{aligned}
$$

Now, integrating over $K_{0}$, overestimating the quantity, and using the density result, we obtain the second continuity result.

The third continuity results holds since

$$
\left|\tau_{\mathrm{i}} w(x)-\tau_{\mathrm{o}} w(x)\right|^{2}=\left|\int_{g_{\mathrm{i}}(x)}^{g_{\mathrm{o}}(x)} \partial_{t} w(x, s) d s\right|^{2} \leq\left(g_{\mathrm{i}}(x)-g_{\mathrm{o}}(x)\right) \int_{g_{\mathrm{i}}(x)}^{g_{\mathrm{o}}(x)}\left|\partial_{t} w(x, s)\right|^{2} d s,
$$

dividing through by $\left(g_{\mathrm{i}}(x)-g_{\mathrm{o}}(x)\right)$ and integrating over $K_{0}$. 
To prove part (ii), we use the following identities

$$
\begin{aligned}
\langle D u, v\rangle_{W} & =\int_{K_{0}}\left(\frac{\tau_{\mathrm{o}} u-\tau_{\mathrm{i}} u}{\left(g_{\mathrm{o}}(x)-g_{\mathrm{i}}(x)\right)^{1 / 2}}\right)\left(\left(g_{\mathrm{o}}(x)-g_{\mathrm{i}}(x)\right)^{1 / 2} \tau_{\mathrm{o}} v\right) d x \\
& +\int_{K_{0}}\left(\frac{\tau_{\mathrm{o}} v-\tau_{\mathrm{i}} v}{\left(g_{\mathrm{o}}(x)-g_{\mathrm{i}}(x)\right)^{1 / 2}}\right)\left(\left(g_{\mathrm{o}}(x)-g_{\mathrm{i}}(x)\right)^{1 / 2} \tau_{\mathrm{i}} u\right) d x
\end{aligned}
$$

and

$$
\begin{aligned}
\langle D u, v\rangle_{W} & =\int_{K_{0}}\left(\frac{\tau_{\mathrm{o}} u-\tau_{\mathrm{i}} u}{\left(g_{\mathrm{o}}(x)-g_{\mathrm{i}}(x)\right)^{1 / 2}}\right)\left(\left(g_{\mathrm{o}}(x)-g_{\mathrm{i}}(x)\right)^{1 / 2} \tau_{\mathrm{i}} v\right) d x \\
& +\int_{K_{0}}\left(\frac{\tau_{\mathrm{o}} v-\tau_{\mathrm{i}} v}{\left(g_{\mathrm{o}}(x)-g_{\mathrm{i}}(x)\right)^{1 / 2}}\right)\left(\left(g_{\mathrm{o}}(x)-g_{\mathrm{i}}(x)\right)^{1 / 2} \tau_{\mathrm{o}} u\right) d x
\end{aligned}
$$

for all $u, v \in W$. These identities holds using the density result and the continuity of the trace theorems proved in part (i). Thus, to prove part (ii) we first show that $V \subseteq\left\{u \in W: \tau_{i}(u)=0\right\}$. Indeed, if $u \in \operatorname{dom}(A)$, then 2.2.6 and 2.2.8a imply

$$
\begin{aligned}
0=\langle D u, v\rangle_{W}= & \int_{0}^{1}\left(\frac{0-\tau_{\mathrm{i}} v}{\left(g_{\mathrm{o}}(x)-g_{\mathrm{i}}(x)\right)^{1 / 2}}\right)\left(\left(g_{\mathrm{o}}(x)-g_{\mathrm{i}}(x)\right)^{1 / 2} \tau_{\mathrm{i}} u\right) \\
& =\int_{0}^{1}\left(-\tau_{\mathrm{i}} v\right)\left(\tau_{\mathrm{i}} u\right) d x, \quad \text { for all } v \in \mathcal{V}^{*}
\end{aligned}
$$

Denoting $\Gamma_{i}=\left\{\left(x, g_{\mathrm{i}}(x)\right): x \in K_{0}\right\}$ and $\Gamma_{0}=\left\{\left(x, g_{\mathrm{o}}(x)\right): x \in K_{0}\right\}$, we observe that $\tau_{i} v \in \mathcal{D}\left(\Gamma_{i}\right)$ for any $v \in \mathcal{V}^{*}$. Since $\mathcal{D}\left(\Gamma_{i}\right)$ is dense in $L^{2}\left(\Gamma_{i}\right)$, we obtain that $\tau_{\mathrm{i}} u=0$ a.e. in $\Gamma_{i}$. Thus, $u \in\left\{w \in W: \tau_{\mathrm{i}}(w)=0\right\}$. For the reverse inclusion let $u \in\{w \in$ $\left.W: \tau_{\mathrm{i}}(w)=0\right\}$. Using again 2.2.8a we conclude that $\langle D u, \phi\rangle_{W}=0$ for all $\phi \in \mathcal{V}^{*}$, thus $u \in \operatorname{dom}(A)$. Thus, $V=\left\{w \in W: \tau_{\mathrm{i}}(w)=0\right\}$. Analogously, using (2.2.8b) we obtain $V^{*}=\left\{v \in W: \tau_{\mathrm{o}}(v)=0\right\}$.

For the third part of the theorem, we prove that $\mathcal{V}^{*}$ is dense in $V^{*}$, since the result $\mathcal{V}$ is dense in $V$ is analogous.

Given any $v \in V^{*}$, by the density of $C^{1}(\bar{K})$ in $W$, there is a sequence $w_{k} \in$ $C^{1}(\bar{K})$ converging to $v$ in $W$. Let $v_{k}(x, t)=w_{k}(x, t)-\left(\tau_{\mathrm{o}} w_{k}\right)(x)$. Since $\tau_{\mathrm{o}}\left(v_{k}\right)=0$, we conclude that $v_{k} \in \mathcal{V}^{*}$, for all $k \in \mathbb{N}$ (definition (2.2.5)). We also know that 
$\partial_{t}\left(\tau\left(w_{k}\right)(x)\right)=0$, thus

$$
\begin{aligned}
\left\|v_{k}-v\right\|_{W} & \leq\left\|w_{k}-v\right\|_{W}+\left\|\tau_{\mathrm{o}} w_{k}\right\|_{L^{2}(K)} \\
& =\left\|w_{k}-v\right\|_{W}+\left(\int_{K_{0}} \int_{g_{\mathrm{i}}(x)}^{g_{\mathrm{o}}(x)}\left|w_{k}(x, x)\right|^{2} d t d x\right)^{1 / 2} \\
& =\left\|w_{k}-v\right\|_{W}+\left\|\tau_{\mathrm{o}} w_{k}\right\|_{L_{g_{\mathrm{o}}(x)-g_{\mathrm{i}}(x)}^{2}\left(\Gamma_{0}\right)} \\
& =\left\|w_{k}-v\right\|_{W}+\left\|\tau_{\mathrm{o}}\left(w_{k}-v\right)\right\|_{L_{g_{\mathrm{o}}(x)-g_{\mathrm{i}}(x)}^{2}\left(\Gamma_{0}\right)} .
\end{aligned}
$$

This, together with trace theorem and the convergence of $w_{k}$ to $v$ in $W$, imply the convergence of $v_{k}$ to $v$ in $W$. Thus $\mathcal{V}^{*}$ is dense in $V^{*}$. Similarly, $\mathcal{V}$ is dense in $V$.

The operator $A$ and $A^{*}$ are bounded by below in their respective domain, with an argument similar to the previous section. Thus all the hypotheses of Theorem 1.4.5 are satisfied for this example, and Theorem 2.2.1 holds.

\subsection{Advection operator on tent-shaped domains}

Definition 2.3.1. Suppose $K$ and $K_{0}$ are as in (2.2.1). If, in addition, $K$ can be divided into finitely many $(d+1)$-simplices with a common edge $\left\{(p, t): g_{\mathrm{i}}(p)<\right.$ $\left.t<g_{\mathrm{o}}(p)\right\}$, then we call $K$ a spacetime tent. We refer to the common edge as its tent pole. Clearly, in this case, $g_{\mathrm{o}}$ and $g_{\mathrm{i}}$ are linear on each simplex of $K_{0}$. We split the tent's boundary into these parts:

$$
\begin{gathered}
\partial_{\mathrm{i}} K=\left\{\left(x, g_{\mathrm{i}}(x)\right): x \in K_{0}\right\}, \quad \partial_{\mathrm{o}} K=\left\{\left(x, g_{\mathrm{o}}(x)\right): x \in K_{0}\right\}, \\
\partial_{\mathrm{b}} K=\partial K \backslash\left(\partial_{\mathrm{i}} K \cup \partial_{\mathrm{o}} K\right) .
\end{gathered}
$$

We refer to the two parts in 2.3.1a as the tent's inflow and outflow boundaries, respectively. (Using such terms without regard to an underlying flow operator is an abuse of terminology that we overlook for expediency.) 


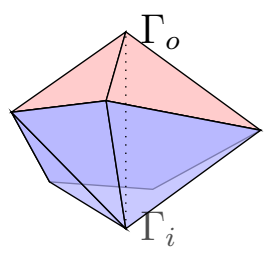

Figure 2.2. Spacetime tent in $\mathbb{R}^{d}$ with $d=3$.

The equation modeling advection along a fixed direction $\alpha \equiv\left(\alpha_{i}\right) \in \mathbb{R}^{d-1}$ is of the form $A u=f$ with

$$
A u=\frac{\partial u}{\partial t}+\sum_{i=1}^{d-1} \alpha_{i} \frac{\partial u}{\partial x_{i}}
$$

Setting $l=m=1$, and noting that $A^{*}=-A$, we can easily see prerequisite (A-a) and $\mathrm{A}-\mathrm{b}$ hold. We define the space $W=\left\{u \in L^{2}(\Omega): A u \in L^{2}(\Omega)\right\}$ and the operator $D: W \rightarrow W^{\prime}$ such that

$$
\langle D u, v\rangle_{W}=(A u, v)+(u, A v) .
$$

We now assume that the tent boundaries are such that

$$
\begin{gathered}
\partial_{\mathrm{i}} K \subseteq\left\{(x, t) \in \partial K: n \text { at }(x, t) \text { satisfies } n_{t}+\alpha \cdot n_{x}<0\right\} \\
\partial_{\mathrm{o}} K \subseteq\left\{(x, t) \in \partial K: n \text { at }(x, t) \text { satisfies } n_{t}+\alpha \cdot n_{x}>0\right\} .
\end{gathered}
$$

The vertical part of the boundary, namely $\partial_{\mathrm{b}} K$, is further split into three parts $\partial_{\mathrm{b}}^{+} K, \partial_{\mathrm{b}}^{-} K$, and $\partial_{\mathrm{b}}^{0} K$ where $n_{t}+\alpha \cdot n_{x}=\alpha \cdot n_{x}$ are positive, negative, and zero, respectively (see Figures 2.2, 2.3). Let $\Gamma_{\mathrm{i}}$ and $\Gamma_{\mathrm{o}}$ denote the closures of $\partial_{\mathrm{i}} K \cup \partial_{\mathrm{b}}^{-} K$ and $\partial_{\mathrm{o}} K \cup \partial_{\mathrm{b}}^{+} K$, respectively, and let

$$
\Gamma_{\mathrm{io}}=\Gamma_{\mathrm{i}} \cap \Gamma_{\mathrm{o}}
$$


Define $\delta(z)=\operatorname{dist}\left(z, \Gamma_{\mathrm{io}}\right)$. We will use the restrictions of this function to $\Gamma_{\mathrm{i}}$ and $\Gamma_{\mathrm{o}}$ as weight functions while describing the norm continuity of traces below. For smooth functions $w$ on $K$, let

$$
\tau_{\mathrm{i}} w=\left.w\right|_{\Gamma_{\mathrm{i}}}, \quad \tau_{\mathrm{o}} w=\left.w\right|_{\Gamma_{\mathrm{o}}} .
$$

We define

$$
\mathcal{V}^{*}=\left\{v \in \mathcal{D}(\bar{K}): \tau_{\mathrm{o}} v=0\right\} \quad \text { and } \quad \mathcal{V}=\left\{v \in \mathcal{D}(\bar{K}): \tau_{\mathrm{i}} v=0\right\}
$$

We set the $\operatorname{dom}(A)$ to be defined as

$$
\operatorname{dom}(A)=\left\{u \in W:\langle D u, v\rangle_{W}=0, \forall v \in \mathcal{V}^{*}\right\}
$$

Note that $\mathcal{D}(K) \subset \operatorname{dom}(A)$, by 2.2 .3 and definition 2.3.5. Thus Assumption $\mathrm{A}-\mathrm{c}$ is satisfied. Then $A$ is a densely defined operator and the domain of the adjoint is given by

$$
\begin{aligned}
\operatorname{dom}\left(A^{*}\right)= & \left\{s \in L^{2}(\Omega): \text { there is an } \ell \in L^{2}(\Omega)\right. \\
& \text { such that } \left.\left(\partial_{t} v, s\right)_{\Omega}=(v, \ell)_{\Omega} \text { for all } v \in \operatorname{dom}(A)\right\} .
\end{aligned}
$$

Note that the operator $D$ is given by:

$$
\langle D u, v\rangle=\int_{\partial K}\left(n_{t} u+\left(\alpha \cdot n_{x}\right) u\right) v, \quad \text { for all } u, v \in \mathcal{D}(\bar{K}) .
$$

Theorem 2.3.2. Let $K$ be a tent and $A$ be given by 2.3.2. Suppose 2.3.3 holds. Then the above-defined maps $\tau_{\mathrm{i}}$ and $\tau_{\mathrm{o}}$ extend to continuous linear operators

$$
\tau_{\mathrm{i}}: W \rightarrow L_{\delta}^{2}\left(\Gamma_{\mathrm{i}}\right) \quad \text { and } \quad \tau_{\mathrm{o}}: W \rightarrow L_{\delta}^{2}\left(\Gamma_{\mathrm{o}}\right)
$$


Hence, setting $V$ and $V^{*}$ as in (1.6.3) and (1.6.4), we can identify these spaces to be $V=\operatorname{ker}\left(\tau_{\mathrm{i}}\right)$ and $V^{*}=\operatorname{ker}\left(\tau_{\mathrm{o}}\right)$, which are closed subspaces of $W$. When restricted to these subspaces, the traces have an additional continuity property, namely

$$
\tau_{\mathrm{i}}: V^{*} \rightarrow L_{1 / \delta}^{2}\left(\Gamma_{\mathrm{i}}\right) \quad \text { and } \quad \tau_{\mathrm{o}}: V \rightarrow L_{1 / \delta}^{2}\left(\Gamma_{\mathrm{o}}\right)
$$

are continuous. Finally, with this $V$ and $V^{*}$, and $\mathcal{V}$ and $\mathcal{V}^{*}$ defined in $(2.3 .4) A s$ sumption 1 is satisfied, and therefore the weak formulation (2.0.1) is well-posed on $K$.

Proof. The idea is to use a change of variable that brings the operator to the previously analyzed operator $\partial_{t}$. The new variables are $\hat{x}=x-\alpha t$ and $\hat{t}=t$, i.e.,

$$
\left[\begin{array}{l}
x \\
t
\end{array}\right]=H\left[\begin{array}{l}
\hat{x} \\
\hat{t}
\end{array}\right] \quad \text { where } \quad H=\left[\begin{array}{ll}
I & \alpha \\
0 & 1
\end{array}\right] .
$$

Let $\hat{K}=H^{-1} K$. (Note that $\hat{K}$ is not a tent, in general.) Pulling back functions $w$ on $K$ to functions $\hat{w}=w \circ H$ on $\hat{K}$, the chain rule gives

$$
\hat{A} \hat{w}=(A w) \circ H, \quad \text { where } \quad \hat{A}=\frac{\partial}{\partial \hat{t}} .
$$

Thus $w \in W$ if and only if $\hat{w} \in \hat{W}=\left\{\hat{z} \in L^{2}(\hat{K}): \hat{A} \hat{z} \in L^{2}(\hat{K})\right\}$.

Next, let $\hat{n}=\left(\hat{n}_{\hat{x}}, \hat{n}_{\hat{t}}\right)$ denote the unit outward normal on $\partial \hat{K}$. Then $\hat{n}=\left(\hat{n}_{\hat{x}}, \hat{n}_{\hat{t}}\right)=$ $H^{t} n /\left\|H^{t} n\right\|_{2}$. Defining $\partial_{\mathrm{i}} \hat{K}, \partial_{\mathrm{o}} \hat{K}$, and $\partial_{\mathrm{c}} \hat{K}$ as in $(2.2 .2)$, we claim that

$$
\begin{aligned}
& \partial_{\mathrm{i}} \hat{K} \equiv\left\{(\hat{x}, \hat{t}) \in \partial \hat{K}: \hat{n}_{\hat{t}}<0\right\}=H^{-1}\left(\partial_{\mathrm{i}} K \cup \partial_{\mathrm{b}}^{-} K\right), \\
& \partial_{\mathrm{o}} \hat{K} \equiv\left\{(\hat{x}, \hat{t}) \in \partial \hat{K}: \hat{n}_{\hat{t}}>0\right\}=H^{-1}\left(\partial_{\mathrm{o}} K \cup \partial_{\mathrm{b}}^{+} K\right), \\
& \partial_{\mathrm{c}} \hat{K} \equiv\left\{(\hat{x}, \hat{t}) \in \partial \hat{K}: \hat{n}_{\hat{t}}=0\right\}=H^{-1}\left(\partial_{\mathrm{b}}^{0} K\right) .
\end{aligned}
$$


To prove the first identity, take any $(x, t) \in \partial_{\mathrm{i}} K$, by definition it will be of the form $\left(x, g_{\mathrm{i}}(x)\right)$. Note that the normal $n$ at $\left(x, g_{\mathrm{i}}(x)\right)$ is in the direction of $\left(\nabla_{x} g_{\mathrm{i}},-1\right)$ where $\nabla_{x}$ denotes the gradient with respect to $x$. Hence, because of (2.3.3), we have $\alpha \cdot \nabla_{x} g_{\mathrm{i}}-1<0$ on $\partial_{\mathrm{i}} \hat{K}$. Since the mapped normal $\hat{n}$ is in the direction of

$$
H^{t} n=\left[\begin{array}{cc}
I & 0 \\
\alpha^{t} & 1
\end{array}\right]\left[\begin{array}{c}
\nabla_{x} g_{\mathrm{i}} \\
-1
\end{array}\right]=\left[\begin{array}{c}
\nabla_{x} g_{\mathrm{i}} \\
\alpha \cdot \nabla_{x} g_{\mathrm{i}}-1
\end{array}\right]
$$

we conclude that $\hat{n}_{\hat{t}}<0$. In the same way, if we take $(x, t) \in \partial_{\mathrm{b}}^{-} K$ the normal vector $n$ at that point will be in the direction of $\left(n_{x}, 0\right)$. By mapping we get

$$
H^{t} n=\left[\begin{array}{cc}
I & 0 \\
\alpha^{t} & 1
\end{array}\right]\left[\begin{array}{c}
n_{x} \\
0
\end{array}\right]=\left[\begin{array}{c}
n_{x} \\
\alpha \cdot n_{x}
\end{array}\right],
$$

hence $\hat{n}_{\hat{t}}<0$ because $\alpha \cdot n_{x}<0$ for all $(x, t) \in \partial_{\mathrm{b}}^{-} K$. Therefore $H^{-1}\left(\partial_{\mathrm{i}} K \cup \partial_{\mathrm{b}}^{-} K\right) \subset$ $\partial_{\mathrm{i}} \hat{K}$. The reverse inclusion has a similar proof using that any normal component $n$ of $\partial K$ goes in the direction of the mapped normal component $\hat{n}$ given by $n=$ $\left(H^{-1}\right)^{t} \hat{n} /\left\|\left(H^{-1}\right)^{t} \hat{n}\right\|_{2}$. Take any $(\hat{x}, \hat{t}) \in \partial_{\mathrm{i}} \hat{K}$, using the mapping we will get

$$
\left(H^{-1}\right)^{t} \hat{n}=\left[\begin{array}{cc}
I & 0 \\
-\alpha^{t} & 1
\end{array}\right]\left[\begin{array}{l}
\hat{n}_{\hat{x}} \\
\hat{n}_{\hat{t}}
\end{array}\right]=\left[\begin{array}{c}
\hat{n}_{\hat{x}} \\
\hat{n}_{\hat{t}}-\alpha \cdot \hat{n}_{\hat{x}}
\end{array}\right] .
$$

Therefore, $n_{t}+\alpha \cdot n_{x}$ goes in the direction of $\hat{n}_{\hat{t}}$, and we conclude $n_{t}+\alpha \cdot n_{x}<0$. If $n_{t}=0, \alpha \cdot n_{x}<0$. Thus $(2.3 .9$ a) holds..

Note that using 2.3.10 we can obtain that $H^{-1}\left(\partial_{\mathrm{b}}^{+} K\right) \subset \partial_{\mathrm{o}} \hat{K}$. Moreover, if we take $(x, t) \in \partial_{\mathrm{o}} K$, the normal component $n$ at that point will be in the direction of $\left(\nabla_{x} g_{\mathrm{o}}, 1\right)$. By mapping we have

$$
H^{t} n=\left[\begin{array}{cc}
I & 0 \\
\alpha^{t} & 1
\end{array}\right]\left[\begin{array}{c}
\nabla_{x} g_{\mathrm{o}} \\
1
\end{array}\right]=\left[\begin{array}{c}
\nabla_{x} g_{\mathrm{o}} \\
1+\alpha \cdot \nabla_{x} g_{\mathrm{o}}
\end{array}\right] .
$$


Therefore $\hat{n}_{\hat{t}}>0$, because $1+\alpha \cdot \nabla_{x} g_{\mathrm{o}}>0$. Hence $H^{-1}\left(\partial_{\mathrm{o}} K \cup \partial_{\mathrm{b}}^{+} K\right) \subset \partial_{\mathrm{o}} \hat{K}$. We conclude as before that $\partial_{\mathrm{o}} \hat{K} \subset H^{-1}\left(\partial_{\mathrm{o}} K \cup \partial_{\mathrm{b}}^{+} K\right)$. Thus $(2.3 .9 \mathrm{~b})$ holds.

From 2.3.10), if $(x, t) \in \partial_{\mathrm{b}}^{0} K$ we have that the normal component at that point satisfies $\alpha \cdot n_{x}=0$, which implies $\hat{n}_{\hat{t}}=0$, and then $H^{-1}\left(\partial_{\mathrm{b}}^{0} K\right) \subset \partial_{\mathrm{c}} \hat{K}$. If we take $(\hat{x}, \hat{t}) \in \partial_{\mathrm{c}} \hat{K}$, we find that the mapped normal component goes in the direction of $\left(\hat{n}_{\hat{x}}, 0\right)$ and have $\partial_{\mathrm{c}} \hat{K} \subset H^{-1}\left(\partial_{\mathrm{b}}^{0} K\right)$. Hence 2.3 .9 c) holds. Thus claim 2.3 .9 is proved.

Let $\hat{K}_{0}$ be the projection of $\hat{K}$ on the $\hat{t}=0$ plane. There are (continuous piecewise linear) functions $\hat{g}_{\mathrm{o}}$ and $\hat{g}_{\mathrm{i}}$ such that $\partial_{\mathrm{o}} \hat{K}$ and $\partial_{\mathrm{i}} \hat{K}$ are graphs of $\hat{g}_{\mathrm{o}}$ and $\hat{g}_{\mathrm{i}}$, respectively, over $\hat{K}_{0}$. On $\hat{K}$, since $\hat{A}=\partial_{\hat{t}}$, we apply Theorem 2.2.1 to conclude that $\hat{\tau}_{\mathrm{i}} \hat{w}=\left.\hat{w}\right|_{\partial_{\mathrm{i}} \hat{K}}$ and $\hat{\tau}_{\mathrm{o}} \hat{w}=\left.\hat{w}\right|_{\partial_{\mathrm{o}} \hat{K}}$ extend to continuous linear operators $\hat{\tau}_{\mathrm{i}}: \hat{W} \rightarrow L_{\hat{g}_{\mathrm{o}}-\hat{g}_{\mathrm{i}}}^{2}\left(\hat{K}_{0}\right)$ and $\hat{\tau}_{\mathrm{o}}: \hat{W} \rightarrow L_{\hat{g}_{\mathrm{o}}-\hat{g}_{\mathrm{i}}}^{2}\left(\hat{K}_{0}\right)$. Hence, setting $\operatorname{dom}(A)$ and $\operatorname{dom}\left(A^{*}\right)$ as in (4.2.2) and 1.6.4), and using Theorem 2.2.1 we let $\hat{V}=\operatorname{ker}\left(\hat{\tau}_{\mathrm{i}}\right)$ and $\hat{V}^{*}=\operatorname{ker}\left(\hat{\tau}_{\mathrm{o}}\right)$ which are closed subspace of $\hat{W}$. By the additional continuity of $\hat{\tau}_{\mathrm{i}}-\hat{\tau}_{\mathrm{o}}: \hat{W} \rightarrow L_{1 /\left(\hat{g}_{\mathrm{o}}-\hat{g}_{\mathrm{i}}\right)}\left(\hat{K}_{0}\right)$ (also given by Theorem 2.2.1, we conclude that

$$
\hat{\tau}_{\mathrm{i}}: \hat{V}^{*} \rightarrow L_{1 /\left(\hat{g}_{\mathrm{o}}-\hat{g}_{\mathrm{i}}\right)}\left(\hat{K}_{0}\right), \quad \text { and } \quad \hat{\tau}_{\mathrm{o}}: \hat{V} \rightarrow L_{1 /\left(\hat{g}_{\mathrm{o}}-\hat{g}_{\mathrm{i}}\right)}\left(\hat{K}_{0}\right)
$$

are also continuous.

These continuity results are more conveniently mapped to $K$ by using $\hat{\delta}(z)=$ $\operatorname{dist}\left(z, \hat{\Gamma}_{\mathrm{io}}\right)$. Note that $\hat{g}_{\mathrm{o}}-\hat{g}_{\mathrm{i}}$ vanishes at $\hat{\Gamma}_{\mathrm{io}}=H^{-1} \Gamma_{\mathrm{io}}$. To restate the continuity properties of $\tau_{\mathrm{i}}$ in terms of $\hat{\delta}$, we prove that there are $c_{1}, c_{2}>0$ such that

$$
c_{1} \hat{\delta}\left(\hat{x}, \hat{g}_{\mathrm{i}}(\hat{x})\right) \leq \hat{g}_{\mathrm{o}}(\hat{x})-\hat{g}_{\mathrm{i}}(\hat{x}) \leq c_{2} \hat{\delta}\left(\hat{x}, \hat{g}_{\mathrm{i}}(\hat{x})\right), \quad \forall \hat{x} \in \hat{K}_{0}
$$

(and similarly for $\tau_{\mathrm{o}}$ ). When a point $N=\left(\hat{x}, \hat{g}_{\mathrm{i}}(\hat{x})\right)$ on $\partial_{\mathrm{i}} \hat{K}$ is sufficiently near to $\hat{\Gamma}_{\mathrm{io}}$, the point $P$ nearest to it on $\hat{\Gamma}_{\mathrm{io}}$, together with $O=\left(\hat{x}, \hat{g}_{\mathrm{o}}(\hat{x})\right)$ form a triangle (as 


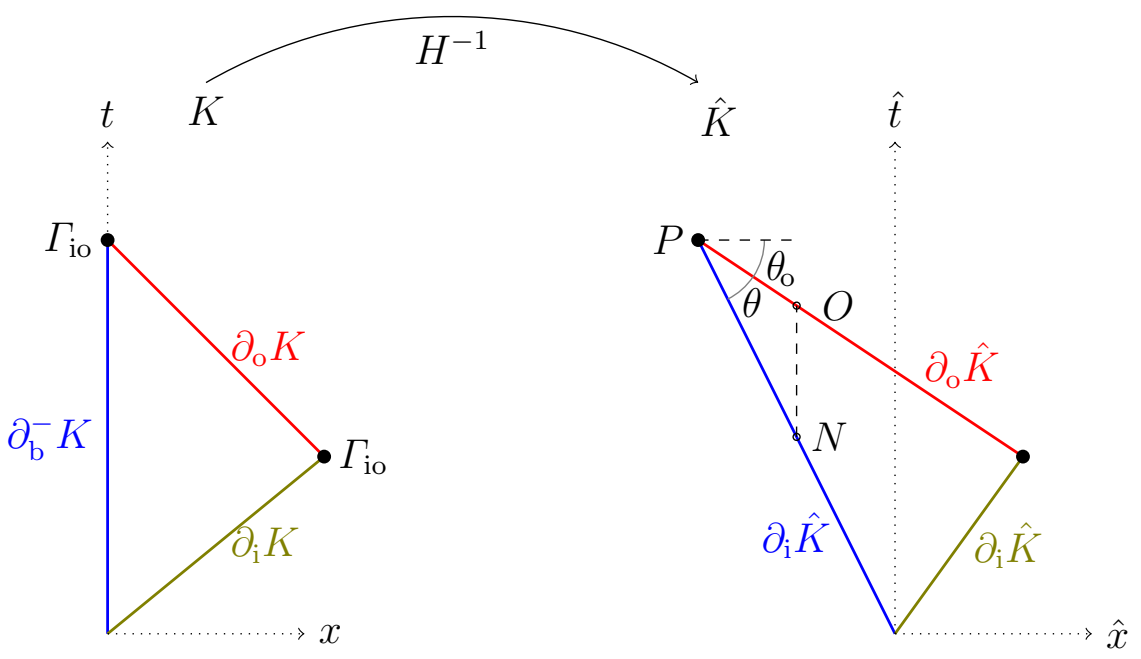

Figure 2.3. On the left is a tent $K$ with $A=\partial_{t}+0.5 \partial_{x}$ that satisfies (2.3.3). On the right is $\hat{K}$ obtained after applying the map in the proof of Theorem 2.3.2 with mapped over operator $\hat{A}=\partial_{\hat{t}}$.

shown in Figure 2.3. Now we may restrict ourselves to the two-dimensional plane containing this triangle.

Consider the case when the segment $P O$ lies on or below the plane of constant $\hat{t}$ passing through $P$, so that $P O$ makes an angle $\theta_{\mathrm{o}} \geq 0$ with that plane. Let $\theta$ be the angle made by $P N$ and $P O$ at $P$. Then, by elementary geometry,

$$
\|P-N\|_{2}=\frac{\cos \theta_{\mathrm{o}}}{\sin \theta}\|O-N\|_{2}
$$

Note that $\theta>0$ and $0 \leq \theta_{0}<\pi / 2$. Therefore, observing that $\|P-N\|_{2}=\hat{\delta}(N)$ and $\|O-N\|_{2}=\hat{g}_{\mathrm{o}}-\hat{g}_{\mathrm{i}}$, 2.3.12) proves (2.3.11). For the remaining geometrical configurations, identities similar to 2.3.12 can be derived to prove 2.3.11). Having established (2.3.11), we find that after mapping back to $K$, the stated continuity properties of $\tau_{\mathrm{i}} w=\left(\hat{\tau}_{\mathrm{i}} \hat{w}\right) \circ H^{-1}$ and $\tau_{\mathrm{o}} w=\left(\hat{\tau}_{\mathrm{o}} \hat{w}\right) \circ H^{-1}$ are proved.

It now only remains to prove the stated wellposedness of the weak formulations. By Theorem 2.2.1, for any $\hat{F} \in \hat{W}^{\prime}$, there is a unique $\hat{u} \in L^{2}(\hat{K})$ and $\hat{q}=\hat{D} \hat{z} \in\left(\hat{V}^{*}\right)^{\perp}$ 
satisfying

$$
-(\hat{u}, \hat{A} \hat{v})_{\hat{K}}+\langle\hat{D} \hat{z}, \hat{v}\rangle_{\hat{W}}=\hat{F}(\hat{v}), \quad \forall \hat{v} \in \hat{W}
$$

where $\hat{D} \in \mathcal{L}\left(\hat{W}, \hat{W}^{\prime}\right)$ is defined as before by $\langle\hat{D} \hat{u}, \hat{v}\rangle_{\hat{W}}=(\hat{A} \hat{u}, \hat{v})_{\hat{K}}+(\hat{u}, \hat{A} \hat{v})_{\hat{K}}$. Here we have used (1.4.4) to find a $\hat{z} \in \hat{V}$ such that $\hat{q}=\hat{D} \hat{z}$. (While $\hat{q}$ is unique, $\hat{z}$ need not be unique.) It now follows from the properties of the mapping that $\hat{u}$ and $\hat{q}=\hat{D} \hat{z}$ satisfies 2.3.13 if and only if $u=\hat{u} \circ H^{-1}$ and $z=\hat{z} \circ H^{-1}$ satisfies

$$
-(u, A v)_{K}+\langle D z, v\rangle_{W}=\hat{F}(v \circ H), \quad \forall v \in W .
$$

Here we have used the fact that 2.3.8) implies $(\hat{u}, \hat{A} \hat{v})_{\hat{K}}=(u, A v)_{K}$ and consequently, $\langle\hat{D} \hat{z}, \hat{v}\rangle_{\hat{W}}=\langle D z, v\rangle_{W}$. This shows that the weak formulations on $\hat{K}$ and $K$ are equivalent, so the well-posedness of the latter, namely (1.4.2), follows from the former.

REMARK 2.3.3. Under additional assumptions, including $\operatorname{dist}\left(\partial_{\mathrm{i}} K, \partial_{\mathrm{o}} K\right)>0$, a stronger trace result is proved in [30, Lemma 5.1]. However, on tents, $\operatorname{dist}\left(\partial_{\mathrm{i}} K, \partial_{\mathrm{o}} K\right)$ is always zero, so we are unable to apply their result.

\subsection{Linear symmetric hyperbolic system}

Let $C \in \mathbb{R}^{m \times m}$ be an $m \times m$ real symmetric matrix and let $K$ be a tent as in Section 2.2. Set $l=m$, and

$$
A u=\frac{\partial u}{\partial t}+\mathrm{C} \frac{\partial u}{\partial x}
$$

where $\partial_{t} u$ and $\partial_{x} u$ are vectors in $\mathbb{R}^{m}$ with their $\ell$ th component equal to $\partial_{t} u_{\ell}$ and $\partial_{x} u_{\ell}$, respectively. Since $\mathrm{C}$ is symmetric, $A^{*}=-A$ and Assumptions (A-a), A-b in 
Chapter 1 are obviously satisfied. Note that

$$
\langle D v, u\rangle=\int_{\partial K}\left(n_{t} v+\mathrm{C} n_{x} \phi\right) \cdot u, \quad \text { for all } \phi, u \in \mathcal{D}(\bar{K})
$$

Thus defining $\operatorname{dom}(A)$ as in 4.2.2, Assumption $\mathrm{A}-\mathrm{c}$ is satisfied. Let $\mathrm{Q}$ be an orthogonal matrix and $\Lambda=\operatorname{diag}\left(\lambda_{\ell}\right)$ be a diagonal matrix such that $\mathrm{C}=\mathrm{Q} \Lambda \mathrm{Q}^{\mathrm{T}}$. Let $\partial_{\mathrm{i}} K, \partial_{\mathrm{o}} K$ and $\partial_{\mathrm{b}} K$ be as defined in 2.3.1). In this subsection, we assume - instead of 2.3 .3 - that

$$
\begin{aligned}
& \partial_{\mathrm{i}} K \subseteq\left\{x \in \partial K: n_{t} I+n_{x} \mathrm{C} \text { is negative definite }\right\} \\
& \partial_{\mathrm{o}} K \subseteq\left\{x \in \partial K: n_{t} I+n_{x} \mathrm{C} \text { is positive definite }\right\}
\end{aligned}
$$

For each $\ell=1, \ldots, m$, we decompose $\partial_{\mathrm{b}} K$ into $\partial_{\mathrm{b}}^{+, \ell} K, \partial_{\mathrm{b}}^{-, \ell} K$, and $\partial_{\mathrm{b}}^{0, \ell} K$ where $\lambda_{\ell} n_{x}$ is positive, negative, and zero, respectively. Then we have the following theorem, which is proved using the diagonalization of $\mathrm{C}$ to separate each component and then appealing to the analysis in Section 2.2. We now opt for a brief statement of the theorem, leaving the tacitly used properties of the traces to the proof.

Theorem 2.4.1. Suppose 2.4.2 holds for the tent $K$ and the operator $A$ in 2.4.1). Then, the formulation 2.0.1 with

$$
\begin{aligned}
V & =\left\{z \in W:\left.\left[\mathrm{Q}^{t} z\right]_{\ell}\right|_{\partial_{\mathrm{i}} K \cup \partial_{\mathrm{b}}^{-, \ell} K}=0, \text { for all } \ell=1, \ldots, m\right\}, \\
V^{*} & =\left\{z \in W:\left.\left[\mathrm{Q}^{t} z\right]_{\ell}\right|_{\partial_{\mathrm{o}} K \cup \partial_{\mathrm{b}}^{+, \ell} K}=0, \text { for all } \ell=1, \ldots, m\right\},
\end{aligned}
$$

is well-posed.

Proof. Let $\breve{A}=Q^{\mathrm{T}} A \mathrm{Q}, \breve{W}=\left\{\breve{v} \in L^{2}(K)^{m}: \breve{A} \breve{v} \in L^{2}(K)^{m}\right\}$, and $\breve{D}$ be the corresponding boundary operator on $\breve{W}$. Then clearly, $v \in W$ if and only if $\breve{v}=\mathrm{Q}^{\mathrm{T}} v$ is in $\breve{W}$, since $\breve{A} \breve{v}=Q^{\mathrm{T}} A v$. Moreover, $\breve{A} \breve{w}=\partial_{t} \breve{w}+\Lambda \partial_{x} \breve{w}$, i.e., its th component 
equals

$$
\breve{A}_{\ell} \breve{w}_{\ell} \equiv \partial_{t} \breve{w}_{\ell}+\lambda_{\ell} \partial_{x} \breve{w}_{\ell}
$$

Note that $\breve{A}_{\ell}$ is an operator of the form $(2.3 .2)$ and has its associated graph space $\breve{W}_{\ell}$ and boundary operator $\breve{D}_{\ell}$.

Now, the assumptions of (2.4.2) imply that 2.3.3) holds for each $\breve{A}_{\ell}\left(\right.$ with $\left.\alpha=\lambda_{\ell}\right)$ so Theorem 2.3.2 yields the continuity of the maps $\breve{\tau}_{\mathrm{i}}^{\ell}:\left.\breve{w} \mapsto \breve{w}_{\ell}\right|_{\Gamma_{\mathrm{i}}^{\ell}}$ and $\breve{\tau}_{\mathrm{o}}^{\ell}:\left.\breve{w} \mapsto \breve{w}_{\ell}\right|_{\Gamma_{\mathrm{o}}^{\ell}}$ on $\breve{W}$, where $\Gamma_{\mathrm{i}}^{\ell}=\partial_{\mathrm{i}} K \cup \partial_{\mathrm{b}}^{-, \ell} K$ and $\Gamma_{\mathrm{o}}^{\ell}=\partial_{\mathrm{o}} K \cup \partial_{\mathrm{b}}^{+, \ell} K$. Therefore, the full trace maps $\breve{\tau}_{\mathrm{i}}=\left(\breve{\tau}_{\mathrm{i}}^{1}, \ldots, \breve{\tau}_{\mathrm{i}}^{m}\right)$ and $\breve{\tau}_{\mathrm{o}}=\left(\breve{\tau}_{\mathrm{o}}^{1}, \ldots, \breve{\tau}_{\mathrm{o}}^{m}\right)$ are continuous on $\breve{W}$. Set $\breve{\mathcal{V}}=\{u \in$ $\left.\mathcal{D}(\bar{K}): \breve{\tau}_{\mathrm{i}} u=0\right\}$ and $\breve{\mathcal{V}}^{*}=\left\{u \in \mathcal{D}(\bar{K}): \breve{\tau}_{\mathrm{o}} u=0\right\}$, thus following Theorem 2.3 .2 we can set $\breve{V}=\operatorname{ker}\left(\breve{\tau}_{\mathrm{i}}\right)$ and $\breve{V}^{*}=\operatorname{ker}\left(\breve{\tau}_{\mathrm{o}}\right)$. Then the following variational equation for $\breve{u} \in L^{2}(K)^{m}$ and $\breve{q}=\breve{D} \breve{z}$ with $\breve{z} \in \breve{V}$,

$$
-(\breve{u}, \breve{A} \breve{v})_{L^{2}(K)^{m}}+\langle\breve{q}, \breve{v}\rangle_{\breve{W}}=F(\breve{v}), \quad \forall \breve{v} \in \breve{W}
$$

splits into $m$ decoupled equations, namely

$$
-\left(\breve{u}_{\ell}, \breve{A}_{\ell} \breve{v}_{\ell}\right)_{L^{2}(K)}+\left\langle\breve{D}_{\ell} \breve{z}_{\ell}, \breve{v}_{\ell}\right\rangle_{\breve{W}_{\ell}}=F\left(\breve{v}_{\ell}\right), \quad \forall \breve{v}_{\ell} \in \breve{W}_{\ell}, \quad \forall \ell=1, \ldots, m .
$$

Here $\breve{u}_{\ell} \in L^{2}(K)$ and $\breve{z}_{\ell} \in \breve{V}_{\ell} \equiv \operatorname{ker}\left(\breve{\tau}_{\mathrm{i}}^{\ell}\right)$ are the $\ell$ th components of $\breve{u}$ and $\breve{z}$, respectively. By Theorem 2.3.2, there is a unique $\breve{u}_{\ell} \in L^{2}(K)$ and $\breve{q}_{\ell}=\breve{D}_{\ell} \breve{z}_{\ell} \in\left(\breve{V}_{\ell}^{*}\right)^{\perp}$ solving (2.4.5) for each $\ell$. This in turn proves the well-posedness of (2.4.4).

To transfer these results for $\breve{A}$ to $A$, we define

$$
\tau_{\mathrm{i}} w=\breve{\tau}_{\mathrm{i}}\left(\mathbf{Q}^{\mathrm{T}} w\right), \quad \tau_{\mathrm{o}} w=\breve{\tau}_{\mathrm{o}}\left(\mathbf{Q}^{\mathrm{T}} w\right) .
$$

Then (2.4.3) is the same as $V=\operatorname{ker}\left(\tau_{\mathrm{i}}\right)$ and $V^{*}=\operatorname{ker}\left(\tau_{\mathrm{o}}\right)$. Note that $z \in V$ if and only if $\breve{z}=\mathrm{Q}^{\mathrm{T}} z \in \breve{V}$. Also note that a $\breve{u} \in L^{2}(K)^{m}$ and $\breve{z} \in \breve{V}$ solves 2.4.4 if and 
only if $u=\mathrm{Q} \breve{u}$ and $z=\mathrm{Q} \breve{z}$ satisfies

$$
-(u, A v)_{K}+\langle D z, v\rangle_{W}=F\left(\mathrm{Q}^{\mathrm{T}} v\right), \quad \forall v \in W .
$$

Here we have used $(\breve{u}, \breve{A} \breve{v})_{K}=(u, A v)_{K}$ and consequent identities for the corresponding boundary operators. Thus the stated well-posedness of 2.0.1 follows from the established well-posedness of 2.4.4).

Remark 2.4.2. Consider a tent $K$ with empty $\partial_{\mathrm{b}} K$. Then, under the assumptions of Theorem 2.4.1, a function in $V$ has all its $m$ components equal to zero on the inflow boundary $\partial_{\mathrm{i}} K$. Moreover, if $v \in \mathcal{V}$, then applying the additional continuity property (2.3.7) to the operators $\breve{\tau}_{\mathrm{o}}^{\ell}$ in the above proof, we find that the outflow trace of each component of $v$ must approach zero as we approach $\Gamma_{\text {io }}$ where the inflow and outflow boundary parts meet. Note that this is an example of 1.6.1, where $\gamma$ is taken as the orthogonal matrix $Q^{T}$. 


\section{A tent pitching scheme for the wave equation}

\subsection{Introduction to tent pitching}

This chapter is dedicated to design an explicit spacetime finite element scheme of a tent pitching type using the spaces and weak formulations motivated by Chapter 2 . The method we construct is a low order method that works on unstructured grids. On uniform grids, comparison with a standard low order finite difference method does not reveal any striking advantages for the new method. However tents of nonuniform heights can be used to handle multiple materials and wavespeeds.

To describe tent pitching schemes in general, consider the case of a hyperbolic problem posed in one space dimension in space and time. Given a spatial mesh, we

pitch a tent by erecting a tent pole (vertically in time) at a vertex, as in Figure 3.1. In the plots of Figure 3.1, the horizontal and vertical dimensions are space and time, respectively. The height of the tent pole must be chosen small enough in relation to the hyperbolic propagation speed, so that the domain of dependence of all points in the tent remains within the tent's footprint. We use the given initial data to solve, by some numerical scheme, the hyperbolic problem restricted to the tent. Proceeding to the next vertex, where the second tent is pitched in Figure 3.1, we find that the initial data combined with the solution in the previous tent, provides inflow data to solve the hyperbolic problem there. Solutions in the newer tents proceeds similarly. 

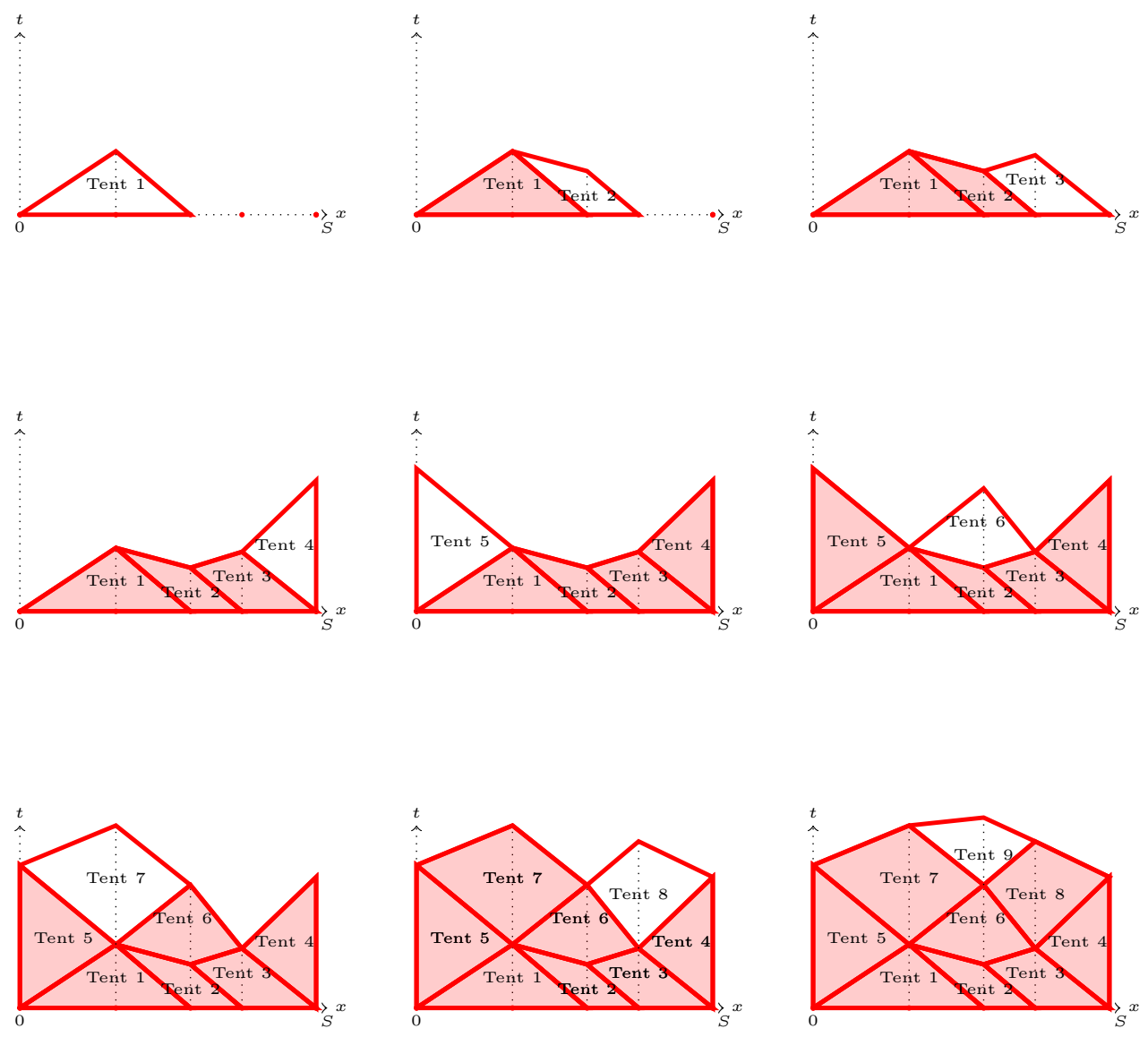

Figure 3.1. Tent pitching mesh (read row by row)

\subsection{Model problem: wave equation}

We now apply the previous ideas introduced in the previous chapter, to the example of the wave equation on spacetime tents and work out the resulting weak formulation in detail. Our model problem is to find a real-valued function $\phi$ on the 
spacetime domain $\Omega=(0, S) \times(0, T)$, satisfying

$$
\begin{aligned}
& c^{-2} \partial_{t t} \phi-\partial_{x x} \phi=g, \quad 0<x<S, 0<t<T, \\
& \partial_{t} \phi=\phi=0, \quad t=0,0<x<S, \\
& \partial_{t} \phi-c \partial_{x} \phi=0, \quad x=0,0<t<T, \\
& \partial_{t} \phi+c \partial_{x} \phi=0, \quad x=S, 0<t<T,
\end{aligned}
$$

where $c>0$ is the wave speed. Here, we have imposed the outgoing impedance boundary conditions (but other boundary conditions can also be considered - see Section 3.5).

The above second order system for $\phi$ arises from a system of first order physical principles, which also matches the form of the problems we have been studying, namely (2.4). Set

$$
u=\left[\begin{array}{c}
c \partial_{x} \phi \\
\partial_{t} \phi
\end{array}\right]
$$

and observe that $\partial_{t} u_{1}=c \partial_{x t} \phi=c \partial_{x} u_{2}$ and $\partial_{t} u_{2}=\partial_{t t} \phi=c \partial_{x} u_{1}+c^{2} g$. These two equations give the first order system $A u=f$ where

$$
A u=\partial_{t} u-\left[\begin{array}{ll}
0 & c \\
c & 0
\end{array}\right] \partial_{x} u, \quad f=\left[\begin{array}{c}
0 \\
c^{2} g
\end{array}\right]
$$

Note that this operator satisfies the conditions (A-a) and A-b after setting $d=l=$ $m=2$ and $k=1$ in Chapter 1. It also fits into the framework of Section 2.4 after the diagonalization

$$
\mathbf{C} \equiv-\left[\begin{array}{ll}
0 & c \\
c & 0
\end{array}\right]=\mathrm{Q} \Lambda \mathbf{Q}^{\mathrm{T}}, \quad \mathbf{Q}=\frac{1}{\sqrt{2}}\left[\begin{array}{rr}
1 & 1 \\
1 & -1
\end{array}\right], \quad \Lambda=\left[\begin{array}{cc}
\lambda_{1} & 0 \\
0 & \lambda_{2}
\end{array}\right]
$$


where $\lambda_{1}=-c$ and $\lambda_{2}=c$. Then, we define $\partial_{\mathrm{i}} \Omega=(0, S) \times\{0\}, \partial_{\mathrm{o}} \Omega=(0, S) \times\{T\}$ and $\partial_{\mathrm{b}} \Omega=\partial \Omega \backslash\left(\partial_{\mathrm{i}} \Omega \cup \partial_{\mathrm{o}} \Omega\right)$. The vertical parts $\partial_{\mathrm{b}} \Omega$ are further split into

$$
\partial_{\mathrm{b}}^{+, 1} \Omega=\partial_{\mathrm{b}}^{-, 2} \Omega=\{0\} \times[0, T], \quad \partial_{\mathrm{b}}^{-, 1} \Omega=\partial_{\mathrm{b}}^{+, 2} \Omega=\{S\} \times[0, T] .
$$

Note that satisfy $\partial_{\mathrm{o}} \Omega, \partial_{\mathrm{i}} \Omega, \partial_{\mathrm{b}}^{+, 1} \Omega=\partial_{\mathrm{b}}^{-, 2} \Omega, \partial_{\mathrm{b}}^{-, 1} \Omega=\partial_{\mathrm{b}}^{+, 2} \Omega$ the previously conditions on a tent $K$ in 2.4.2. Indeed, since

$$
n=\left(n_{x}, n_{t}\right)=\left\{\begin{array}{cc}
(0,-1) & \text { if }(x, t) \in \partial_{\mathrm{i}} \Omega, \\
(0,1) & \text { if }(x, t) \in \partial_{\mathrm{o}} \Omega, \\
(-1,0) & \text { if }(x, t) \in\{0\} \times[0, T], \\
(1,0) & \text { if }(x, t) \in\{S\} \times[0, T],
\end{array}\right.
$$

then

$$
\begin{aligned}
& \partial_{\mathrm{o}} \Omega \subseteq\left\{(x, t) \in \partial \Omega: n_{t} I+C n_{x} \text { is negative definite }\right\}, \\
& \partial_{\mathrm{o}} \Omega \subseteq\left\{(x, t) \in \partial \Omega: n_{t} I+C n_{x} \text { is positive definite }\right\}, \\
& \partial_{\mathrm{b}}^{+, 1} \Omega \subseteq\left\{(x, t) \in \partial \Omega: \lambda_{1} n_{x}=-c n_{x}>0\right\}, \\
& \partial_{\mathrm{b}}^{-, 1} \Omega \subseteq\left\{(x, t) \in \partial \Omega: \lambda_{1} n_{x}=-c n_{x}<0\right\}, \\
& \partial_{\mathrm{b}}^{+, 2} \Omega \subseteq\left\{(x, t) \in \partial \Omega: \lambda_{2} n_{x}=c n_{x}>0\right\}, \\
& \partial_{\mathrm{b}}^{-, 2} \Omega \subseteq\left\{(x, t) \in \partial \Omega: \lambda_{2} n_{x}=c n_{x}<0\right\} .
\end{aligned}
$$

Following Theorem 2.4.1, to understand the wave operator, it is sufficient to understand the diagonalized (decoupled) operator:

$$
\breve{A} \breve{w}=\partial_{t} \breve{w}+\Lambda \partial_{x} \breve{w}
$$




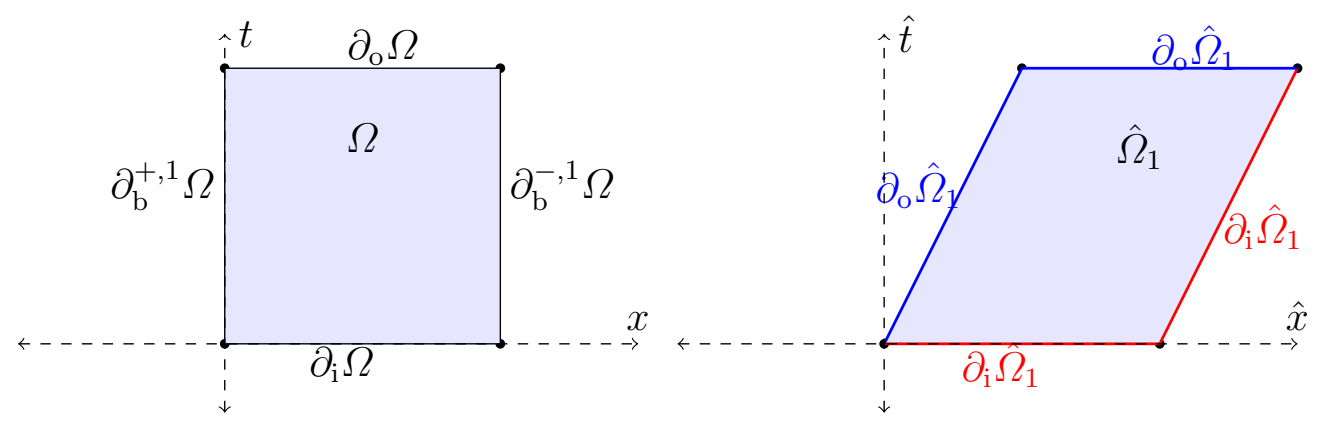

Figure 3.2. Mapping $\Omega$ to $\hat{\Omega}_{1}$ using the $H_{1}$ transformation.

Then, we pull back $\ell$ - component of the decoupled vector function using the mapping

$$
H_{\ell}=\left[\begin{array}{cc}
1 & (-1)^{\ell} c \\
0 & 1
\end{array}\right]
$$

Following Theorem 2.4.1, set $\Gamma_{\mathrm{i}}^{\ell}$ and $\Gamma_{\mathrm{o}}^{\ell}$ to the closures of $\partial_{\mathrm{i}} \Omega \cup \partial_{\mathrm{b}}^{-, \ell} \Omega$ and $\partial_{\mathrm{o}} \Omega \cup \partial_{\mathrm{b}}^{+, \ell} \Omega$, respectively, $\Gamma_{\mathrm{io}}^{\ell}=\Gamma_{\mathrm{i}}^{\ell} \cap \Gamma_{\mathrm{o}}^{\ell}$, and $\delta_{\ell}(x, t)=\operatorname{dist}\left((x, t), \Gamma_{\mathrm{io}}^{\ell}\right)$ for $\ell=1,2$. We set the graph space $W=\left\{u \in L^{2}(\Omega)^{2}: A u \in L^{2}(\Omega)^{2}\right\}$, and the operator $D$ as before. Note that the conditions in $(2.3 .9)$ holds for each component and its mapping operator $H_{\ell}$. Indeed,

$$
\begin{gathered}
\partial_{\mathrm{i}} \hat{\Omega}_{\ell} \equiv\left\{(\hat{x}, \hat{t}) \in \partial \hat{\Omega}_{\ell}: \hat{n}_{\hat{t}}<0\right\}=H_{\ell}^{-1}\left(\partial_{\mathrm{i}} \Omega \cup \partial_{\mathrm{b}}^{-, 1} \Omega\right) \\
\partial_{\mathrm{o}} \hat{\Omega}_{\ell} \equiv\left\{(\hat{x}, \hat{t}) \in \partial \hat{\Omega}_{\ell}: \hat{n}_{\hat{t}}>0\right\}=H_{\ell}^{-1}\left(\partial_{\mathrm{o}} \Omega \cup \partial_{\mathrm{b}}^{+, 1} \Omega\right),
\end{gathered}
$$

Note that $\hat{\Omega}_{1}$ is a parallelogram with vertices $\{(0,0),(S, 0),(S+c T, T),(c T, T)\}$, and $\hat{\Omega}_{2}$ is the parallelogram with vertices $\{(0,0),(S, 0),(S-c T, T),(-c T, T)\}$. See Figure 3.2 the mapping of the first component of the decoupled vector function.

Thus, defining

$$
\tau_{\mathrm{i}} w=\breve{\tau}_{\mathrm{i}}\left(\mathbf{Q}^{\mathrm{T}} w\right), \quad \tau_{\mathrm{o}} w=\breve{\tau}_{\mathrm{o}}\left(\mathbf{Q}^{\mathrm{T}} w\right)
$$


we can prove, as in Section 2.4, that the global trace maps

$$
\begin{aligned}
& \tau_{\mathrm{i}}\left[\begin{array}{c}
z_{1} \\
z_{2}
\end{array}\right]=\left[\begin{array}{l}
\left.\left(\mathrm{Q}^{t} u\right)_{1}\right|_{\Gamma_{\mathrm{i}}^{1}} \\
\left.\left(\mathrm{Q}^{t} u\right)_{2}\right|_{\Gamma_{\mathrm{i}}^{2}}
\end{array}\right]=\left[\begin{array}{l}
\left.\left(z_{1}+z_{2}\right)\right|_{\Gamma_{\mathrm{i}}^{1}} \\
\left.\left(z_{1}-z_{2}\right)\right|_{\Gamma_{\mathrm{i}}^{2}}
\end{array}\right], \quad \tau_{\mathrm{i}}: W \rightarrow L_{\delta_{1}}^{2}\left(\Gamma_{\mathrm{i}}^{1}\right) \times L_{\delta_{2}}^{2}\left(\Gamma_{\mathrm{i}}^{2}\right) \\
& \tau_{\mathrm{o}}\left[\begin{array}{c}
z_{1} \\
z_{2}
\end{array}\right]=\left[\begin{array}{l}
\left.\left(\mathrm{Q}^{t} u\right)_{1}\right|_{\Gamma_{\mathrm{o}}^{1}} \\
\left.\left(\mathrm{Q}^{t} u\right)_{2}\right|_{\Gamma_{\mathrm{o}}^{2}}
\end{array}\right]=\left[\begin{array}{l}
\left.\left(z_{1}+z_{2}\right)\right|_{\Gamma_{\mathrm{o}}^{1}} \\
\left.\left(z_{1}-z_{2}\right)\right|_{\Gamma_{\mathrm{o}}^{2}}
\end{array}\right], \quad \tau_{\mathrm{o}}: W \rightarrow L_{\delta_{1}}^{2}\left(\Gamma_{\mathrm{o}}^{1}\right) \times L_{\delta_{2}}^{2}\left(\Gamma_{\mathrm{o}}^{2}\right)
\end{aligned}
$$

are continuous, and we can set

$$
V(\Omega)=\operatorname{ker}\left(\tau_{\mathrm{i}}\right), \quad V^{*}(\Omega)=\operatorname{ker}\left(\tau_{\mathrm{o}}\right),
$$

Indeed, since $\tau_{\mathrm{i}} w=\breve{\tau}_{\mathrm{i}} \breve{w}$ and $\tau_{\mathrm{o}} w=\breve{\tau}_{\mathrm{o}} \breve{w}$ in (3.2.3), we just need to study the components of the decoupled operator $\breve{\tau_{\mathrm{i}}} \breve{w}$, i.e. $\left(\breve{\tau_{\mathrm{i}}} \breve{w}\right)_{\ell}$. Mapping $\Omega$ to $\hat{\Omega}_{\ell}$ for $\ell=1,2$, by

$$
\left(\breve{\tau}_{\mathrm{i}} \breve{w}\right)_{\ell}=\left(\hat{\tau}_{\mathrm{i}} \hat{w}_{\ell}\right) \circ H_{\ell}^{-1} \quad \text { and } \quad\left(\breve{\tau_{\mathrm{o}}} \breve{w}\right)_{\ell}=\left(\hat{\tau}_{\mathrm{o}} \hat{w}_{\ell}\right) \circ H_{\ell}^{-1}
$$

we can prove that $\tau_{\mathrm{i}} w$ satisfies the continuity properties by proving that operator on $\hat{\delta}_{\ell}$ on $\hat{\Omega}_{\ell}$, satisfies

$$
c_{1}^{\ell} \hat{\delta}_{\ell}\left(\hat{x}, \hat{g}_{\mathrm{i}}^{\ell}(\hat{x})\right) \leq \hat{g}_{\mathrm{o}}^{\ell}(\hat{x})-\hat{g}_{\mathrm{i}}^{\ell}(\hat{x}) \leq c_{2}^{\ell} \hat{\delta}_{\ell}\left(\hat{x}, \hat{g}_{\mathrm{i}}^{\ell}(\hat{x})\right)
$$

for $c_{1}^{\ell}, c_{2}^{\ell}$ constants. Then, the continuity properties of the operator $\hat{\tau}_{\mathrm{i}}: \hat{W} \rightarrow$ $L_{\hat{g}_{\mathrm{o}}-\hat{g}_{\mathrm{i}}}^{2}\left(\hat{K}_{0}\right)$ showed in Theorem 2.2.1) will also imply the continuity properties for the operator $\left(\breve{\tau_{\mathrm{i}}} \breve{w}\right)_{\ell}$, after mapping back to $\Omega$, and therefore for $(3.2 .4)$. The proof of the continuity properties of $\tau_{\mathrm{o}}$ is analogous.

Since the steps to find the constant $c_{1}^{\ell}$ and $c_{2}^{\ell}$ for $l=1,2$ are similar, we just show the case when $\ell=1$. Since $\hat{\Gamma}_{\text {io }}^{1}=\{(0,0),(S+c T, T)\}$, if we choose a point $(\hat{x}, 0)$ closed to $(0,0)$, we obtain $c_{1}^{1}=c_{2}^{1}=\frac{1}{c}$. If we choose $\left(\hat{x}, \frac{1}{c}(\hat{x}-S)\right)$ approaching $(S+c T, T)$ we obtain $\hat{g}_{\mathrm{o}}^{1}(\hat{x})-\hat{g}_{\mathrm{i}}^{1}(\hat{x})=(c T+S-\hat{x}) / c$, and $\hat{\delta}_{1}\left(\hat{x}, \hat{g}_{\mathrm{i}}^{1}(\hat{x})\right)=(c T+S-\hat{x}) \sqrt{c^{2}+1} / c$, 
thus $c_{1}^{1}=c_{2}^{1}=\frac{1}{c^{2}+1}$. See Figure 3.2. Analogously we obtain similar results with $\hat{\Gamma}_{\text {io }}^{2}=\{(-c T, T),(S, 0)\}$, and therefore the trace continuity properties are well defined for the wave equation in $\Omega$.

These spaces can be used to give a global weak formulation on $\Omega$, but our focus is on local solvers. Note that $\mathcal{D}(\Omega)^{2} \subset V(\Omega)$, and therefore condition A-c is satisfied.

In spacetime tent pitching methods, we are required to numerically solve the wave equation on spacetime tents, ordered so that inflow data on a tent can be provided by the outflow solution on previously handled tents or through given data. Hence we now focus on the formulation and discretization on one tent $K$.

\subsection{Weak formulation on a tent}

Consider the analogue of $(3.2 .1)$ on one tent $K$, with zero initial data on the inflow boundaries and with boundary conditions inherited from the global boundary conditions $3.2 .1 \mathrm{c}-3.2 .1 \mathrm{~d}$.

Define, as before, the boundary parts of a tent $K$, by

$$
\begin{gathered}
\partial_{\mathrm{i}} K=\left\{(x, t) \in \partial K: n_{t}<0\right\}, \quad \partial_{\mathrm{o}} K=\left\{(x, t) \in \partial K: n_{t}>0\right\}, \quad \partial_{\mathrm{b}} K=\partial K \backslash\left(\partial_{\mathrm{i}} K \cup \partial_{\mathrm{o}} K\right), \\
\partial_{\mathrm{b}}^{+, 1} K=\partial_{\mathrm{b}}^{-, 2} K=\left\{(x, t) \in \partial_{\mathrm{b}} K: c n_{x}<0\right\}, \\
\partial_{\mathrm{b}}^{+, 2} K=\partial_{\mathrm{b}}^{-, 1} K=\left\{(x, t) \in \partial_{\mathrm{b}} K: c n_{x}>0\right\} .
\end{gathered}
$$

Note that the boundary part $\partial_{\mathrm{b}} K$ may be empty in some tents. We consider the tent problem of solving for $u$ satisfying

$$
\begin{aligned}
& A u=f \quad \text { on } K, \quad u_{1}-u_{2}=0 \quad \text { on } \partial_{\mathrm{b}}^{+, 1} K \text {, } \\
& u=0 \quad \text { on } \partial_{\mathrm{i}} K, \quad u_{1}+u_{2}=0 \quad \text { on } \partial_{\mathrm{b}}^{+, 2} K \text {. }
\end{aligned}
$$

To obtain a well-posed weak formulation on one tent, we proceed to use Theorem 2.4.1. 
To this end, we must assume that the tent satisfies Assumption 2.4.2, which now reads

$$
\begin{gathered}
\partial_{\mathrm{i}} K \subseteq\left\{x \in \partial K: n_{t} \pm n_{x} c<0\right\} \\
\partial_{\mathrm{o}} K \subseteq\left\{x \in \partial K: n_{t} \pm n_{x} c>0\right\}
\end{gathered}
$$

Since $A^{*}=-A$ in this example, the weak formulation 2.0 .1 reads

$$
u \in L, q \in Q: \quad-(u, A v)_{L}+\langle q, v\rangle_{W}=F(v), \quad \forall v \in W
$$

where the spaces are set following (2.4.3), namely

$$
\begin{gathered}
V=\left\{\left[\begin{array}{l}
z_{1} \\
z_{2}
\end{array}\right] \in W:\left.\left[\begin{array}{l}
z_{1}+z_{2} \\
z_{1}-z_{2}
\end{array}\right]_{\ell}\right|_{\partial_{\mathrm{i}} K \cup \partial_{\mathrm{b}}^{-, \ell} K}=0, \quad \text { for } \ell=1,2\right\}, \\
V^{*}=\left\{\left[\begin{array}{c}
z_{1} \\
z_{2}
\end{array}\right] \in W:\left.\left[\begin{array}{l}
z_{1}+z_{2} \\
z_{1}-z_{2}
\end{array}\right]_{\ell}\right|_{\partial_{\mathrm{o}} K \cup \partial_{\mathrm{b}}^{+, \ell} K}=0, \quad \text { for } \ell=1,2\right\} .
\end{gathered}
$$

Theorem 2.4.1 shows that 3.3.2 is a well-posed weak formulation on $K$ provided the tent $K$ satisfies (3.3.1). Note that the above spaces change from tent to tent and may arguably be better denoted by $V(K), V^{*}(K)$, etc., but to avoid notational bulk we will suppress the $K$-dependence.

3.3.1. Causality condition. Let us take a closer look at (3.3.1). First note that each tent, in this application, consists of either two triangles (on either side of the tent pole), or just one triangle. The tents are thus divided into three types, as shown in Figure 3.3 .

The length of the tent pole is $k$, the numbers $p_{l}$ and $p_{r}$ are such that $p_{l} k$ and $p_{r} k$ give the heights of the outflow boundaries on the left and right side of the tent pole, respectively, and the spatial mesh size are $h_{r}, h_{l} \geq 0$. Writing down the normal 


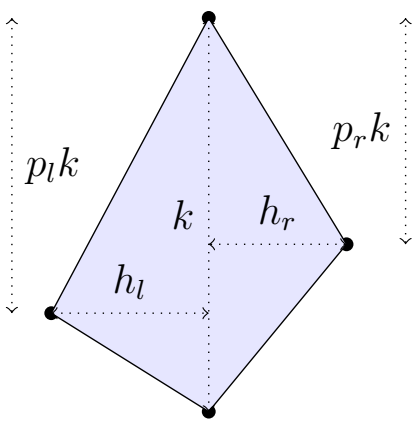

Type I: $h_{r}>0, h_{l}>0$

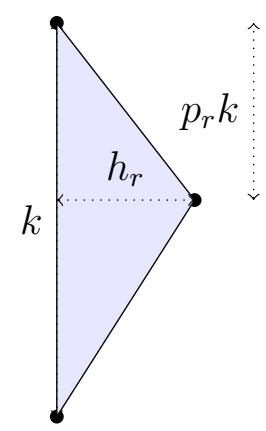

Type II: $h_{r}>0, h_{l}=0$

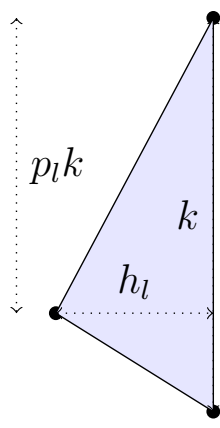

Type III: $h_{r}=0, h_{l}>0$

Figure 3.3. Three types of tents

vector on the tent boundaries, we immediately find that condition (3.3.1) on a tent is equivalent to

$$
\left|c \frac{k p_{r}}{h_{r}}\right|<1 \quad \text { and } \quad\left|c \frac{k p_{l}}{h_{l}}\right|<1 .
$$

Clearly, by controlling the size of the tent pole we can satisfy these inequalities.

The well-known Courant-Friedrichs-Levy (CFL) condition [14] identifies stability conditions as constraints on the time step size in terms of space mesh size in numerical discretizations. In our case, an analogue manifests itself as geometrical constraints (3.3.3) on the tent. We will refer to (3.3.1) - or (3.3.3) - as the causality condition. 


\subsection{The numerical scheme}

In this section, continuing to work with the wave operator $A$ defined by (3.2.2), we give an explicit numerical scheme for approximating $u(x, t)$ satisfying

$$
\begin{aligned}
A u & =f, & & 0<x<S, 0<t<T, \\
u_{i}(x, 0) & =u_{i}^{0}(x), & & 0<x<S, i=1,2, \\
u_{1}(0, t)-u_{2}(0, t) & =0, & & 0<t<T, \\
u_{1}(1, t)+u_{2}(1, t) & =0, & & 0<t<T .
\end{aligned}
$$

The scheme will allow varying spatial and temporal mesh sizes. Here $f$ and $u^{0}$ are assumed to given smooth functions. We begin by describing the calculations within each tent, followed by the tent pitching technique to advance in time.

3.4.1. Conforming discretization on a tent. As seen above, a tent is comprised of one or two triangles. Let the space of continuous functions on a tent $K$ whose restrictions to these triangles are linear be denoted by $P_{1}^{h}(K)$. We construct a conforming discretization of 3.3 .2 within $K$ using the discrete space

$$
V_{1}=V \cap\left(P_{1}^{h}(K)\right)^{2}
$$

By definition, $V_{1} \subseteq V$, and consequently, functions in $V_{1}$ must satisfy the essential boundary conditions of $V$. Depending on the tent geometry, different boundary conditions must be imposed on different tents.

To examine what this entails for the nodal coefficients on mesh vertices, let $\zeta \in$ $P_{1}^{h}(K)$ be the continuous scalar function (unique Lagrange basis function) that equals one at the "apex" of the tent $K$, equals zero at all its other vertices. The apex of a tent, irrespective of whether it consists of one or two triangles, is the vertex in $\partial_{\mathrm{o}} K$ 
that is away from $\partial_{\mathrm{i}} K$. Now, suppose $\mu \equiv\left[\begin{array}{l}\mu_{1} \\ \mu_{2}\end{array}\right]$ in $\mathbb{R}^{2}$ is such that

$$
\begin{array}{ll}
\mu_{1}-\mu_{2}=0 & \text { if } \partial_{\mathrm{b}}^{+, 1} K \text { is nonempty, } \\
\mu_{1}+\mu_{2}=0 & \text { if } \partial_{\mathrm{b}}^{-, 1} K \text { is nonempty. }
\end{array}
$$

(Note that if $\partial_{\mathrm{b}} K$ is empty, then $\mu$ is an arbitrary vector in $\mathbb{R}^{2}$.) Then, it is easy to see that

$$
V_{1}=\{\mu \zeta: \mu \text { satisfies } 3.4 .3\}
$$

provides an alternate characterization of (3.4.2). Indeed, if $K$ of type I (see Figure 3.3. If $u \in V(K)$, then $u \in P_{1}^{h}(K)^{2}$. We then recall $\zeta$, and denote $\zeta_{b}, \zeta_{l}$ and $\zeta_{r}$ the basis functions that equals one at the bottom, left, and right respectively, and zero in the other vertices. Then, $u=\mu \zeta+\mu_{b} \zeta_{b}+\mu_{l} \zeta_{l}+\mu_{r} \zeta_{r}$, for $\mu, \mu_{b}, \mu_{l}, \mu_{r}$ in $\mathbb{R}^{2}$ (for the other two tent types, we omit one of the terms associated to the missing vertex). Since $u$ is also in $V$, then $\left.(\mathrm{Q} u)\right|_{\partial_{\mathrm{i}} K}=0$, thus $\mu_{b}, \mu_{l}, \mu_{r}=0$ and therefore $u=\mu \zeta$ for any $\mu \in \mathbb{R}^{2}$. If $K$ is of type II, then we also have $u \in V_{1}$ is given by $u=\mu \zeta$, since $\left.(\mathrm{Q} u)\right|_{\partial_{\mathrm{i}} K}=0$, but since $\partial_{\mathrm{b}}^{-, 1} K$ is not empty, we have $(\mathrm{Q} u)_{1}=\left(\mu_{1}+\mu_{2}\right) \zeta=0$ at $\partial_{\mathrm{b}}^{-, 1} K$ thus $\mu$ satisfies condition (3.4.3). Analogously, if $K$ is of type III, then $u \in V^{1}$ implies that there exists $\mu \in \mathbb{R}^{2}$, such that $(\mathrm{Q} u)_{2}=\left(\mu_{1}-\mu_{2}\right) \zeta=0$ at $\partial_{\mathrm{b}}^{-, 2} K$, that equals $\partial_{\mathrm{b}}^{+, 1} K$, thus $\mu$ satisfies condition 3.4 .3 .

A computable conforming discretization of 3.3.2 additionally requires finitedimensional subspaces of $L^{2}(K)^{2}$ and $Q$. For the latter, observe that when looking to $D\left(V_{1}\right)$ as a functional into the dual space of $W_{h}$, we have

$$
D\left(V_{1}\right) \subset D(V)=D_{h, V}=Q
$$


Hence we choose an approximation $q_{1}$ of the solution component $q$ in (3.3.2) to have the form

$$
q_{1}=D z_{1}, \quad z_{1} \in V_{1} .
$$

Then $q_{1}$ is clearly in $Q$. Next, set $L_{1} \subset L^{2}(K)^{2}$ to be the space of vector functions whose components are constant functions on $K$. Finally, set

$$
W_{1}=\left\{w: w=\kappa+\mu \zeta, \quad \kappa, \mu \in \mathbb{R}^{2}, \quad \kappa \zeta \in V_{1}\right\} .
$$

Our discretization of (3.3.2) now takes the following form: Find $u_{1}$ in $L_{1}$ and $q_{1} \in D\left(V_{1}\right)$ satisfying $-\left(u_{1}, A w\right)+\left\langle q_{1}, w\right\rangle=F(w)$, for all $w \in W_{1}$. Clearly, $\operatorname{dim}\left(W_{1}\right)$ is four or three, depending on whether $\partial_{\mathrm{b}} K$ is empty or not. This equation gives rise to an invertible discrete system, as a consequence of the unisolvency of the following slightly modified problem:

Find $u_{1} \in L_{1}$ and $z_{1} \in V_{1}$ such that

$$
-\left(u_{1}, A w\right)+\left\langle D z_{1}, w\right\rangle=F(w), \quad \forall w \in W_{1} .
$$

Proposition 3.4.1. There is a unique solution for Problem (3.4.6).

Proof. Note the $\operatorname{dim}\left(L_{1}\right)+\operatorname{dim}\left(V_{1}\right)=\operatorname{dim}\left(W_{1}\right)$, so 3.4 .6 gives a square (PetrovGalerkin) system. Hence it suffices to set $F=0$ and prove that $u_{1}=z_{1}=0$. With $F=0$, writing $z_{1}=\alpha \zeta$ for some $\alpha \in \mathbb{R}^{2}$, we have $\langle D(\alpha \zeta), w\rangle=0$ for all constant $w \in W$. Since $\alpha \zeta \in V_{1}$, by the definition of $W_{1}$, we may set $w=\alpha$ in (3.4.6), to get

$$
\langle D(\alpha \zeta), \alpha\rangle=\int_{\partial_{\mathrm{o}} K \cup \partial_{\mathrm{b}} K} \mathrm{D}_{\mathrm{x}, \mathrm{t}} \alpha \cdot \alpha \zeta=0
$$

where

$$
\mathrm{D}_{\mathrm{x}, \mathrm{t}}=n_{t} I+\mathrm{C} n_{x}=\left[\begin{array}{cc}
n_{t} & -c n_{x} \\
-c n_{x} & n_{t}
\end{array}\right] \text {. }
$$


If $\partial_{\mathrm{b}} K$ is empty, then since $\mathrm{D}_{\mathrm{x}, \mathrm{t}} \alpha \cdot \alpha=\left(\alpha_{1}+\alpha_{2}\right)^{2}\left(n_{t}-c n_{x}\right) / 2+\left(\alpha_{1}-\alpha_{2}\right)^{2}\left(n_{t}+c n_{x}\right) / 2$, the causality condition (3.3.1) gives $\alpha=0$. If $\partial_{\mathrm{b}} K$ is nonempty, then whenever $\alpha \zeta \in V$ we have either $\alpha_{1}-\alpha_{2}=0$ or $\alpha_{1}+\alpha_{2}=0$, so we can continue to conclude that $\alpha=0$. Of course $\alpha=0$ implies $z_{1}=0$.

To prove that $u_{1}=0$, we use 1.2 .3 after substituting $z_{1}=0$, to get

$$
\left\langle D w, u_{1}\right\rangle=0, \quad \forall w \in W_{1} .
$$

Since $u_{1}$ is a constant function, $u_{1} \zeta \in W_{1}$, so we may choose $w=u_{1} \zeta$ and conclude that $u_{1}=0$ by an argument analogous to what we used above.

REMARK 3.4.2. One can view $\left.z_{1}\right|_{\partial_{\mathrm{o}} K}$ as an interface trace variable and $q_{1}=D z_{1}$ as an interface flux variable. By the trace theory we developed previously, outflow trace $\left.z_{1}\right|_{\partial_{\mathrm{o}} K}$ must vanish at the points where outflow and inflow edges meet in order for $z_{1}$ to be in $V$. This motivates our choice (3.4.4) of $V_{1}$ to obtain a conforming method. Other non-conforming avenues to design approximations within a tent can be found in 33 and 50 .

3.4.2. Advancing in time by tent pitching. We now show how the above ideas yield an explicit time marching algorithm for solving (3.4.1).

First, we mesh the spacetime domain $\Omega=(0, S) \times(0, T)$ by a collection $\Omega_{h}$ of tents $K$ with these properties: The first property is that either $\partial_{\mathrm{b}} K$ is empty or

$$
\partial_{\mathrm{b}} K \subseteq \partial_{\mathrm{b}} \Omega
$$

for all $K \in \Omega_{h}$. Second, there exists an enumeration of all tents, $K_{1}, K_{2}, \ldots, K_{J}$, with the property that for each $j \in\{1, \ldots J\}$,

$$
\partial_{\mathrm{i}} K_{j} \subseteq \bigcup_{k=1}^{j-1} \partial_{\mathrm{o}} K_{k} \cup \partial_{\mathrm{i}} \Omega
$$


Finally, for all $j \in\{1, \ldots J\}$,

$$
K_{j} \text { satisfies the causality condition (3.3.3). }
$$

It is well-known how to construct an algorithm (not only in one space dimension, but also in higher dimensions [29, 67]) that produces meshes satisfying (3.4.8), so we shall not dwell further on the meshing process.

The discrete spacetime approximation on the mesh $\Omega_{h}$ is developed using

$$
V_{h}=\left\{z \in H^{1}(\Omega)^{2} \cap V(\Omega):\left.z\right|_{K} \in P_{1}^{h}(K)^{2}, \forall K \in \Omega_{h}, z(x, 0)=I_{h} u^{0}(x)\right\}
$$

$$
L_{h}=\left\{\alpha:\left.\alpha\right|_{K} \in \mathbb{R}^{2} \text { is constant on each } K \in \Omega_{h}\right\}
$$

$$
W_{h}=\left\{w:\left.w\right|_{K} \in W_{1} \text { on each } K \in \Omega_{h}\right\},
$$

where $I_{h}$ denote the linear nodal interpolant on the spatial mesh. The method finds approximations $u_{h} \in L_{h}$ and $z_{h} \in V_{h}$ satisfying

$$
\sum_{K \in \Omega_{h}}\left(-\int_{K} u_{h} \cdot A w+\int_{\partial K} \mathrm{D}_{\mathrm{x}, \mathrm{t}} z_{h} \cdot w\right)=\sum_{K \in \Omega_{h}} \int_{K} f \cdot w, \quad \forall w \in W_{h}
$$

where $\mathrm{D}_{\mathrm{x}, \mathrm{t}}$ is as in 3.4 .7 .

Because of 3.4.8), we are able to use a time-marching algorithm to solve 3.4.10): Proceed in the ordering of (3.4.8b), and for each tent $K$, solve for $\left.u_{h}\right|_{K}$ and $\left.z_{h}\right|_{K}$. Specifically, if $\alpha$ is the nodal (vector) value of $z_{h}$ at the apex of $K$, then defining $z_{\mathrm{o}}^{K}=\alpha \zeta$, the problem on one tent is to find $\left.u_{h}\right|_{K} \in L_{1}$ and $z_{\mathrm{o}}^{K} \in V_{1}$ satisfying (3.4.10), namely

$$
-\int_{K} u_{h} \cdot A w+\int_{\partial K} \mathrm{D}_{\mathrm{x}, \mathrm{t}} z_{\mathrm{o}}^{K} \cdot w=\int_{K} f \cdot w-\int_{\partial K} \mathrm{D}_{\mathrm{x}, \mathrm{t}} z_{\mathrm{i}}^{K} \cdot w, \quad \forall w \in W_{1} .
$$

where $z_{\mathrm{i}}^{K}=z_{h}-z_{\mathrm{o}}^{K}$. Note that $z_{\mathrm{i}}^{K}$ on right hand side will be a known quantity if $3.4 .8 \mathrm{~b}$ holds and if we have already solved on every $K^{\prime}$ appearing before $K$ in 
the ordering of tents in (3.4.8). Indeed, $z_{\mathrm{i}}^{K}$ is completely determined by its nodal values at (the three or two) vertices on $\partial_{\mathrm{i}} K$, which either lie at $t=0$ or were apex vertices of previous tents. Problem (3.4.11) is exactly of the same type we discussed in Section 3.4.1.

3.4.3. Propagation formula. Since the system 3.4 .11 is small, we can explicitly calculate its solution. To see how information is propagated from inflow to outflow on a mesh of tents, we consider the case where the volume source $f$ is zero. Write $z_{h}=\left[\begin{array}{l}z_{h, 1} \\ z_{h, 2}\end{array}\right]$ in (3.4.10) and let the nodal values of the scalar Lagrange finite element functions $z_{h, 1}$ and $z_{h, 2}$ be $\left[\begin{array}{l}U^{t} \\ V^{t}\end{array}\right],\left[\begin{array}{c}U^{b} \\ V^{b}\end{array}\right],\left[\begin{array}{c}U^{l} \\ V^{l}\end{array}\right],\left[\begin{array}{c}U^{r} \\ V^{r}\end{array}\right]$, at the top, bottom, left and right vertices, respectively, of a tent of Type I, as in Figure 3.3. For the other two tent types, we omit the nodal values at the missing vertex.

Equation 3.4.11 finds $\left[\begin{array}{c}U^{t} \\ V^{t}\end{array}\right]$ as a function of the remaining nodal values. After tedious simplifications (displayed below), this relationship is found to be as follows:

$$
\begin{aligned}
& {\left[\begin{array}{l}
U^{t} \\
V^{t}
\end{array}\right]=\left[\begin{array}{l}
U^{b} \\
V^{b}
\end{array}\right]+w_{1}\left[\begin{array}{ll}
0 & c \\
c & 0
\end{array}\right]\left[\begin{array}{l}
U^{r}-U^{l} \\
V^{r}-V^{l}
\end{array}\right]+w_{2} c\left[\begin{array}{l}
U^{r}-U^{l} \\
V^{r}-V^{l}
\end{array}\right], \quad \text { for Type I, }} \\
& {\left[\begin{array}{l}
U^{t} \\
V^{t}
\end{array}\right]=\left[\begin{array}{l}
U^{b} \\
V^{b}
\end{array}\right]+w_{1}\left[\begin{array}{ll}
0 & c \\
c & 0
\end{array}\right]\left[\begin{array}{l}
U^{r}-U^{b} \\
V^{r}-V^{b}
\end{array}\right]+w_{2} c\left[\begin{array}{l}
U^{r}-U^{b} \\
V^{r}-V^{b}
\end{array}\right],} \\
& {\left[\begin{array}{l}
U^{t} \\
V^{t}
\end{array}\right]=\left[\begin{array}{l}
U^{b} \\
V^{b}
\end{array}\right]+w_{1}\left[\begin{array}{ll}
0 & c \\
c & 0
\end{array}\right]\left[\begin{array}{l}
U^{b}-U^{l} \\
V^{b}-V^{l}
\end{array}\right]+w_{2} c\left[\begin{array}{l}
U^{b}-U^{l} \\
V^{b}-V^{l}
\end{array}\right],}
\end{aligned}
$$


where

$$
\begin{array}{lll}
w_{1}=\frac{\left(h_{r}+h_{l}\right) k}{\left(h_{r}+h_{l}\right)^{2}-c^{2} k^{2}\left(p_{r}-p_{l}\right)^{2}}, & w_{2}=\frac{c k^{2}\left(p_{r}-p_{l}\right)}{\left(h_{r}+h_{l}\right)^{2}-c^{2} k^{2}\left(p_{r}-p_{l}\right)^{2}}, & \text { for Type I, } \\
w_{1}=\frac{k}{2\left(c k\left(1-p_{r}\right)+h_{r}\right)}, & w_{2}=w_{1}, & \text { for Type II, } \\
w_{1}=\frac{k}{2\left(c k\left(1-p_{l}\right)+h_{l}\right)}, & w_{2}=-w_{1}, & \text { for Type III. }
\end{array}
$$

Indeed:

Tent of type I. We consider a tent of type I, with vertices at $(0,0),\left(h_{r}, k-\right.$ $\left.p_{r} k\right),(k, 0)$ and $\left(-h_{l}, k-p_{l} k\right)$, and define $K_{r}$ the triangular domain with vertices $(0,0),\left(h_{r}, k-p_{r} k\right),(k, 0)$ and $K_{l}$ the triangular domain defined by $(0,0),(k, 0)$ and $\left(-h_{l}, k-p_{l} k\right)$ as in Figure 3.4. Since $L^{1} \subset L^{2}(K)^{2}$ is the space of constant vector functions, we choose as basis $\left\{\eta_{1}=\left[\begin{array}{l}1 \\ 0\end{array}\right], \eta_{2}=\left[\begin{array}{l}0 \\ 1\end{array}\right]\right\}$. We define

$$
\zeta(x, t)= \begin{cases}\frac{1}{k}\left(t-\frac{k\left(1-p_{r}\right)}{h_{r}} x\right) & \text { if }(x, t) \in K_{r}, \\ \frac{1}{k}\left(\left(t+\frac{k\left(1-p_{l}\right)}{h_{l}} x\right)\right. & \text { if }(x, t) \in K_{l} .\end{cases}
$$

This function is one at the apex, zero at other vertices, and linear along the edges. See Figure 3.5. Thus, the space $V_{1}$ can be generated by the vector functions $\nu_{1}=\left[\begin{array}{c}\zeta(x, t) \\ 0\end{array}\right]$ and $\nu_{2}=\left[\begin{array}{c}0 \\ \zeta(x, t)\end{array}\right]$, and the space $W_{1}$ defined in 3.4.5) can be generated by the basis $\left\{\chi_{1}=\left[\begin{array}{l}1 \\ 0\end{array}\right], \chi_{2}=\left[\begin{array}{l}0 \\ 1\end{array}\right], \chi_{3}=\left[\begin{array}{c}\zeta(x, t) \\ 0\end{array}\right], \chi_{4}=\left[\begin{array}{c}0 \\ \zeta(x, t)\end{array}\right]\right\}$.

Note that $A\left(w_{1}\right)=A\left(w_{2}\right)=\left[\begin{array}{l}0 \\ 0\end{array}\right]$ and

$$
\begin{aligned}
& A\left[\begin{array}{l}
\zeta \\
0
\end{array}\right]=\left\{\left[\begin{array}{c}
\frac{1}{k} \\
c \frac{1-p_{r}}{h_{r}}
\end{array}\right] \text { if }(x, t) \in K_{r}, \text { and }\left[\begin{array}{c}
\frac{1}{k} \\
-c \frac{1-p_{l}}{h_{l}}
\end{array}\right] \text { if }(x, t) \in K_{l}\right\}, \\
& A\left[\begin{array}{l}
0 \\
\zeta
\end{array}\right]=\left\{\left[\begin{array}{c}
c \frac{1-p_{r}}{h_{r}} \\
\frac{1}{k}
\end{array}\right] \text { if }(x, t) \in K_{r}, \text { and }\left[\begin{array}{c}
-c \frac{1-p_{l}}{h_{l}} \\
\frac{1}{k}
\end{array}\right] \text { if }(x, t) \in K_{l}\right\} .
\end{aligned}
$$

Now we compute the left hand side. We define

$$
\left[\mathrm{B}_{0}\right]_{\mathrm{ij}}=-\int_{K} \eta_{j} A\left(\chi_{i}\right) \text { and }\left[\mathrm{B}_{1}\right]_{\mathrm{ij}}=\int_{\partial K} \mathrm{D}_{\mathrm{x}, \mathrm{t}} \nu_{j} \cdot \chi_{i} \text {. }
$$


Since the entries of the first two rows of $B_{0}$ are zero, we only display

$$
\begin{aligned}
{\left[\mathrm{B}_{0}\right]_{31} } & =-\int_{K}\left[\begin{array}{l}
1 \\
0
\end{array}\right] \cdot A\left[\begin{array}{l}
\zeta \\
0
\end{array}\right]=-\int_{K} \frac{1}{k}=-\frac{h_{r}+h_{l}}{2} \\
{\left[\mathrm{~B}_{0}\right]_{32} } & =-\int_{K}\left[\begin{array}{l}
0 \\
1
\end{array}\right] \cdot A\left[\begin{array}{l}
\zeta \\
0
\end{array}\right]=\int_{K_{l}} c \frac{1-p_{l}}{h_{l}}-\int_{K_{r}} c \frac{1-p_{r}}{h_{r}}=\frac{c k\left(p_{r}-p_{l}\right)}{2}, \\
{\left[\mathrm{~B}_{0}\right]_{41} } & =-\int_{K}\left[\begin{array}{l}
1 \\
0
\end{array}\right] \cdot A\left[\begin{array}{l}
0 \\
\zeta
\end{array}\right]=\int_{K_{l}} c \frac{1-p_{l}}{h_{l}}-\int_{K_{r}} c \frac{1-p_{r}}{h_{r}}=\left[\mathrm{B}_{0}\right]_{32}, \\
{\left[\mathrm{~B}_{0}\right]_{42} } & =-\int_{K}\left[\begin{array}{l}
0 \\
1
\end{array}\right] \cdot A\left[\begin{array}{l}
0 \\
\zeta
\end{array}\right]=-\int_{K} \frac{1}{k}=\left[\mathrm{B}_{0}\right]_{31} .
\end{aligned}
$$

Next, the calculation of the outward normal vector $n$ at different parts of the boundary gives us the following result

$$
\left.n=n_{x}, n_{t}\right)=\left\{\begin{array}{cc}
\frac{1}{\sqrt{h_{r}^{2}+p_{r}^{2} k^{2}}}\left(p_{r} k, h_{r}\right) & \text { if }(x, t) \in \partial_{\mathrm{o}} K_{r}, \\
\frac{1}{\sqrt{h_{l}^{2}+p_{l}^{2} k^{2}}}\left(-p_{l} k, h_{l}\right) & \text { if }(x, t) \in \partial_{\mathrm{o}} K_{l}, \\
\frac{1}{\sqrt{h_{r}^{2}+\left(k-p_{r} k\right)^{2}}}\left(k-p_{r} k,-h_{r}\right) & \text { if }(x, t) \in \partial_{\mathrm{i}} K_{r}, \\
\frac{1}{\sqrt{h_{l}^{2}+\left(k-p_{l} k\right)^{2}}}\left(p_{l} k-k,-h_{l}\right) & \text { if }(x, t) \in \partial_{\mathrm{i}} K_{l} .
\end{array}\right.
$$

We introduce the functions $r_{o}(x)=\left(x, g_{\mathrm{o}}(x)\right)$ and $r_{i}(x)=\left(x, g_{\mathrm{i}}(x)\right)$, where

$$
g_{\mathrm{o}}(x)=\left\{\begin{array}{cl}
\frac{p_{r} k}{h_{r}} x+k & \text { if } 0<x<h_{r}, \\
-\frac{p_{l} k}{h_{l}} x+k & \text { if } 0<x<h_{l},
\end{array} \quad \text { and } \quad g_{\mathrm{i}}(x)=\left\{\begin{array}{cl}
\frac{k-p_{r} k}{h_{r}} x & \text { if } 0<x<h_{r}, \\
-\frac{k-p_{l} k}{h_{l}} x & \text { if } 0<x<h_{l}
\end{array}\right.\right.
$$

are functions introduced to parametrize the segments $\partial_{\mathrm{o}} K_{r}$ and $\partial_{\mathrm{o}} K_{l}$. From the definition of $\zeta$, it is easy to see that $\zeta\left(x, g_{\mathrm{o}}(x)\right)= \begin{cases}1-\frac{x}{h_{r}} & \text { if } 0<x<h_{r}, \\ 1+\frac{x}{h_{l}} & \text { if } h_{l}<x<0 .\end{cases}$

Since $\zeta$ vanishes at $\partial_{\mathrm{i}} K$, then

$$
\left[\mathrm{B}_{1}\right]_{\mathrm{ij}}=\int_{\partial_{\mathrm{o}} K_{r}} \mathrm{D}_{\mathrm{x}, \mathrm{t}} \nu_{j} \cdot \chi_{i}+\int_{\partial_{\mathrm{o}} K_{l}} \mathrm{D}_{\mathrm{x}, \mathrm{t}} \nu_{j} \cdot \chi_{i}
$$


Since $D_{x, t}$ is a constant matrix when restricted to specific parts of the boundary,

$$
\int_{\partial_{\mathrm{o}} K_{r}} \mathrm{D}_{\mathrm{x}, \mathrm{t}} \nu_{j} \cdot \chi_{i}=\int_{0}^{h_{r}} \frac{1}{h_{r}}\left[\begin{array}{cc}
h_{r} & -c p_{r} k \\
-c p_{r} k & h_{r}
\end{array}\right] \nu_{j}\left(r_{o}(x)\right) \cdot \chi_{i}\left(r_{o}(x)\right) d x
$$

and

$$
\int_{\partial_{\mathrm{o}} K_{l}} \mathrm{D}_{\mathrm{x}, \mathrm{t}} \nu_{j} \cdot \chi_{i}=\int_{-h_{l}}^{0} \frac{1}{h_{l}}\left[\begin{array}{cc}
h_{l} & c p_{l} k \\
c p_{l} k & h_{l}
\end{array}\right] \nu_{j}\left(r_{o}(x)\right) \cdot \chi_{i}\left(r_{o}(x)\right) d x .
$$

Note that the values of $n_{x}$ and $n_{t}$ are the values of the components of the normal vector of $\partial_{\mathrm{o}} K_{r}$ and $\partial_{\mathrm{o}} K_{l}$ respectively, see (3.4.13). Then,

$$
\begin{aligned}
& {\left[\mathrm{B}_{1}\right]_{11}=\left[\mathrm{B}_{1}\right]_{22}=\int_{0}^{h_{r}}\left(1-\frac{x}{h_{r}}\right) d x+\int_{-h_{l}}^{0}\left(1+\frac{x}{h_{l}},\right) d x=\frac{h_{l}+h_{r}}{2},} \\
& {\left[\mathrm{~B}_{1}\right]_{12}=\left[\mathrm{B}_{1}\right]_{21}=\int_{0}^{h_{r}} \frac{-c p_{r} k}{h_{r}}\left(1-\frac{x}{h_{r}}\right) d x+\int_{-h_{l}}^{0} \frac{c p_{l} k}{h_{l}}\left(1+\frac{x}{h_{l}}\right)=\frac{c k}{2}\left(p_{l}-p_{r}\right),} \\
& {\left[\mathrm{B}_{1}\right]_{31}=\left[\mathrm{B}_{1}\right]_{42}=\int_{0}^{h_{r}}\left(1-\frac{x}{h_{r}}\right)^{2} d x+\int_{-h_{l}}^{0}\left(1+\frac{x}{h_{l}}\right)^{2} d x=\frac{h_{l}+h_{r}}{3},} \\
& {\left[\mathrm{~B}_{1}\right]_{32}=\left[\mathrm{B}_{1}\right]_{41}=\int_{0}^{h_{r}} \frac{-c p_{r} k}{h_{r}}\left(1-\frac{x}{h_{r}}\right)^{2} d x+\int_{-h_{l}}^{0} \frac{c p_{l} k}{h_{l}}\left(1+\frac{x}{h_{l}}\right)^{2}=\frac{c k}{3}\left(p_{l}-p_{r}\right) .}
\end{aligned}
$$

Thus,

$$
\mathrm{B}_{0}=\left[\begin{array}{cc}
0 & 0 \\
0 & 0 \\
-\frac{h_{r}+h_{l}}{2} & \frac{c k\left(p_{r}-p_{l}\right)}{2} \\
\frac{c k\left(p_{r}-p_{l}\right)}{2} & -\frac{h_{r}+h_{l}}{2}
\end{array}\right] \quad \text { and } \quad \mathrm{B}_{1}=\left[\begin{array}{cc}
\frac{h_{r}+h_{l}}{2} & -\frac{c k\left(p_{r}-p_{l}\right)}{2} \\
-\frac{c k\left(p_{r}-p_{l}\right)}{2} & \frac{h_{r}+h_{l}}{2} \\
\frac{h_{r}+h_{l}}{3} & -\frac{c k\left(p_{r}-p_{l}\right)}{3} \\
-\frac{c k\left(p_{r}-p_{l}\right)}{3} & \frac{h_{r}+h_{l}}{3}
\end{array}\right]
$$

We define $B=\left[B_{0}, B_{1}\right]$. 
Moreover, we can write $\mathrm{B}=\left[\begin{array}{cc}0 & \frac{1}{2} M \\ \frac{-1}{2} M & \frac{1}{3} M\end{array}\right]$, by setting
\[ \mathrm{M}=\left[\begin{array}{cc}h_{r}+h_{l} & -c k\left(p_{r}-p_{l}\right) \\ -c k\left(p_{r}-p_{l}\right) & h_{r}+h_{l}\end{array}\right] . \]

Thus,

$$
\mathrm{B}^{-1}=\left[\begin{array}{cc}
\frac{4}{3} M^{-1} & -2 M^{-1} \\
2 M^{-1} & 0
\end{array}\right], \quad \text { where } \quad \mathrm{M}^{-1}=\frac{1}{\operatorname{det}(\mathrm{M})}\left[\begin{array}{cc}
h_{r}+h_{l} & c k\left(p_{r}-p_{l}\right) \\
c k\left(p_{r}-p_{l}\right) & h_{r}+h_{l}
\end{array}\right]
$$

and $\operatorname{det}(\mathrm{M})=\left(h_{r}+h_{l}\right)^{2}-c^{2} k^{2}\left(p_{r}-p_{l}\right)^{2}$.

To calculate the right hand side we choose

$$
\begin{aligned}
& \zeta_{r}(x, t)=\left\{\begin{array}{cl}
\frac{x}{h_{r}} & \text { if } x \in K_{r}, \\
0 & \text { if } x \in K_{l} .
\end{array}, \quad \zeta_{l}(x, t)=\left\{\begin{array}{cl}
-\frac{x}{h_{l}} & \text { if } x \in K_{l}, \\
0 & \text { if } x \in K_{r}
\end{array}\right. \text { and }\right. \\
& \zeta_{b}(x, t)=\left\{\begin{array}{cl}
-\frac{p_{r}}{h_{r}} x+1-\frac{t}{k} & \text { if }(x, t) \in K_{r}, \\
\frac{p_{l}}{h_{l}} x+1-\frac{t}{k} & \text { if }(x, t) \in K_{l} .
\end{array}\right.
\end{aligned}
$$

From the structure of matrix $B^{-1}$, it is easy to see that

$$
\left[\begin{array}{l}
U^{t} \\
V^{t}
\end{array}\right]=2 M^{-1}\left[\begin{array}{l}
F_{1} \\
F_{2}
\end{array}\right]
$$

where, following 3.4.11,

$$
\begin{aligned}
& F_{1}=-\int_{\partial K} \mathrm{D}_{\mathrm{x}, \mathrm{t}}\left(\left[\begin{array}{l}
U_{b} \\
V_{b}
\end{array}\right] \zeta_{b}+\left[\begin{array}{l}
U_{l} \\
V_{l}
\end{array}\right] \zeta_{l}+\left[\begin{array}{l}
U_{r} \\
V_{r}
\end{array}\right] \zeta_{r}\right) \cdot\left[\begin{array}{l}
1 \\
0
\end{array}\right], \\
& F_{2}=-\int_{\partial K} \mathrm{D}_{\mathrm{x}, \mathrm{t}}\left(\left[\begin{array}{l}
U_{b} \\
V_{b}
\end{array}\right] \zeta_{b}+\left[\begin{array}{l}
U_{l} \\
V_{l}
\end{array}\right] \zeta_{l}+\left[\begin{array}{l}
U_{r} \\
V_{r}
\end{array}\right] \zeta_{r}\right) \cdot\left[\begin{array}{l}
0 \\
1
\end{array}\right] .
\end{aligned}
$$




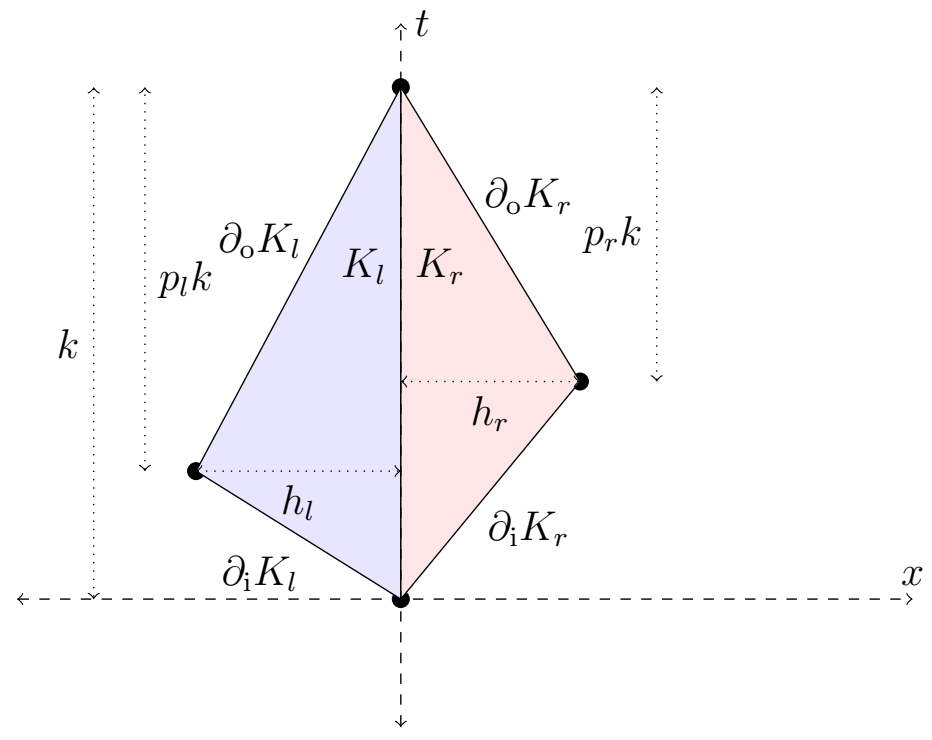

Figure 3.4. Type I: $h_{r}>0, h_{l}>0$

We use the following results

$$
\begin{aligned}
& -\int_{\partial K} \mathrm{D}_{\mathrm{x}, \mathrm{t}}\left[\begin{array}{l}
U_{i} \\
V_{i}
\end{array}\right] \cdot\left[\begin{array}{l}
1 \\
0
\end{array}\right]=-\int_{\partial K} n_{t} U_{i}-c \int_{\partial_{\mathrm{o}} K} n_{x} V_{i}, \\
& -\int_{\partial K} \mathrm{D}_{\mathrm{x}, \mathrm{t}}\left[\begin{array}{l}
U_{i} \\
V_{i}
\end{array}\right] \cdot\left[\begin{array}{l}
0 \\
1
\end{array}\right]=-\int_{\partial_{\mathrm{i}} K} n_{t} U_{i}-c \int_{\partial_{\mathrm{i}} K} n_{x} V_{i}
\end{aligned}
$$

for $i=b, l, r$ to define and calculate

$$
S_{i}=\left[\begin{array}{cc}
\int_{\partial K} n_{t} \zeta_{i} & -c \int_{\partial K} n_{x} \zeta_{i} \\
-c \int_{\partial K} n_{x} \zeta_{i} & \int_{\partial K} n_{t} \zeta_{i}
\end{array}\right], \quad \text { for } i=b, l, r .
$$

Thus, we can rewrite 3.4.14 as follows

$$
\left[\begin{array}{c}
F_{1} \\
F_{2}
\end{array}\right]=S_{b}\left[\begin{array}{l}
U_{b} \\
V_{b}
\end{array}\right]+S_{l}\left[\begin{array}{l}
U_{l} \\
V_{l}
\end{array}\right]+S_{r}\left[\begin{array}{l}
U_{r} \\
V_{r}
\end{array}\right] .
$$


Since $\zeta_{b}\left(x, g_{i}(x)\right)=\left\{\begin{array}{ll}1-\frac{x}{h_{r}} & \text { if } 0<x<h_{r} \\ 1+\frac{x}{h_{l}} & \text { if } h_{l}<x<0\end{array}\right.$, we calculate:

$$
\begin{aligned}
\int_{\partial K} n_{t} \zeta_{b} & =\int_{-h_{l}}^{0}\left(1+\frac{x}{h_{l}}\right) d x+\int_{0}^{h_{r}}\left(1-\frac{x}{h_{r}}\right) d x=\frac{h_{l}+h_{r}}{2} \\
\int_{\partial K} n_{x} \zeta_{b} & =\frac{k-k p_{r}}{h_{r}} \int_{0}^{h_{r}}\left(1+\frac{x}{h_{r}}\right) d x+\frac{p_{l} k-k}{h_{l}} \int_{-h_{l}}^{0}\left(1+\frac{x}{h_{l}}\right) d x=\frac{k\left(p_{l}-p r\right)}{2} \\
\int_{\partial K_{r}} n_{t} \zeta_{r} & =\int_{\partial K_{l}} n_{t} \zeta_{l}=0 \\
\int_{\partial K_{r}} n_{x} \zeta_{r} & =-\int_{\partial K_{l}} n_{x} \zeta_{l}=\frac{k}{2}
\end{aligned}
$$

to find the matrices $S_{i}$. Hence,

$$
\left[\begin{array}{l}
F_{1} \\
F_{2}
\end{array}\right]=\frac{1}{2} M\left[\begin{array}{l}
U_{b} \\
V_{b}
\end{array}\right]+\left[\begin{array}{ll}
0 & k \\
k & 0
\end{array}\right]\left[\begin{array}{l}
U_{l}-U_{r} \\
V_{l}-V_{r}
\end{array}\right]
$$

Multiplying by $\mathrm{M}^{-1}$ to the left, we obtain the desired result. Indeed,

$$
\left[\begin{array}{l}
U_{t} \\
V_{t}
\end{array}\right]=\left[\begin{array}{l}
U_{b} \\
V_{b}
\end{array}\right]+M^{-1}\left[\begin{array}{ll}
0 & k \\
k & 0
\end{array}\right]\left[\begin{array}{l}
U_{l}-U_{r} \\
V_{l}-V_{r}
\end{array}\right]
$$

equals 3.4.12.

Tent of Type II. We denote $K_{r}=K$ to present the results for tents of Type II in 3.4.12). We consider the basis of $V_{1}$ to be $\nu_{1}=\left[\begin{array}{l}\zeta(x, t) \\ \zeta(x, t)\end{array}\right]$. Thus, a basis for $W_{1}$ is given by $\left\{\chi_{1}=\left[\begin{array}{l}1 \\ 1\end{array}\right], \chi_{2}=\left[\begin{array}{c}\zeta(x, t) \\ 0\end{array}\right], \chi_{3}=\left[\begin{array}{c}0 \\ \zeta(x, t)\end{array}\right]\right\}$, where $\zeta=\frac{1}{k}\left(t-\frac{k\left(1-p_{r}\right)}{h_{r}} x\right)$. As before, the basis functions for $L^{1} \subset L^{2}\left(K_{r}\right)$ are chosen to be $\left\{\eta_{1}=\left[\begin{array}{l}1 \\ 0\end{array}\right], \eta_{2}=\left[\begin{array}{l}0 \\ 1\end{array}\right]\right\}$. Defining

$$
\left[\mathrm{B}_{0}\right]_{\mathrm{ij}}=-\int_{K_{r}} \eta_{j} A\left(\chi_{i}\right) \text { and }\left[\mathrm{B}_{1}\right]_{\mathrm{ij}}=\int_{\partial K_{r}} \mathrm{D}_{\mathrm{x}, \mathrm{t}} \nu_{j} \cdot \chi_{i}
$$




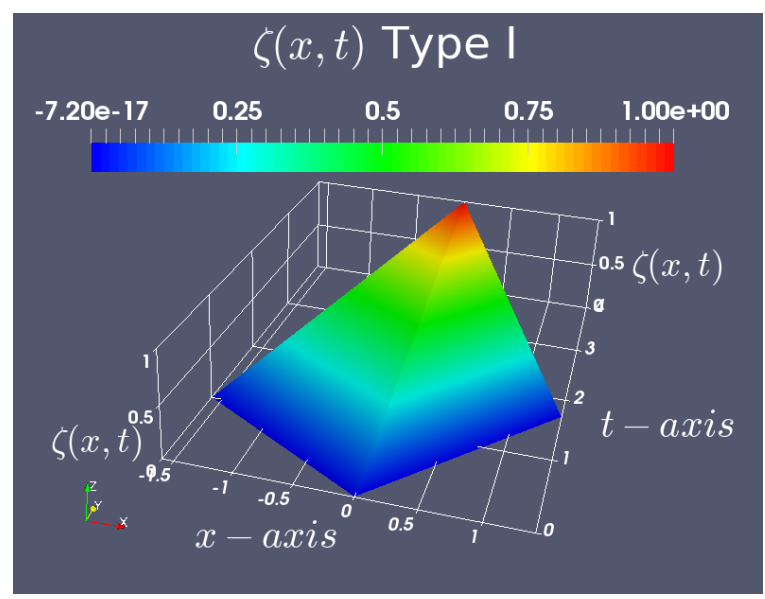

Figure 3.5. $\zeta(x, t)$ basis function for tent of Type I

as before, we obtain

$$
\begin{aligned}
& {\left[\mathrm{B}_{0}\right]_{21}=-\int_{K_{r}}\left[\begin{array}{l}
1 \\
0
\end{array}\right] \cdot A\left[\begin{array}{l}
\zeta \\
0
\end{array}\right]=-\int_{K_{r}} \frac{1}{k}=-\frac{h_{r}}{2}} \\
& {\left[\mathrm{~B}_{0}\right]_{22}=-\int_{K_{r}}\left[\begin{array}{l}
0 \\
1
\end{array}\right] \cdot A\left[\begin{array}{l}
\zeta \\
0
\end{array}\right]=-\int_{K_{r}} c \frac{1-p_{r}}{h_{r}}=\frac{c k\left(p_{r}-1\right)}{2},} \\
& {\left[\mathrm{~B}_{0}\right]_{31}=\left[\mathrm{B}_{0}\right]_{22} \quad \text { and } \quad\left[\mathrm{B}_{0}\right]_{32}=\left[\mathrm{B}_{0}\right]_{31}}
\end{aligned}
$$

and

$$
\begin{aligned}
{\left[\mathrm{B}_{1}\right]_{11} } & =\int_{\partial_{\mathrm{o}} K_{r} \cup \partial_{\mathrm{b}}^{+, 1} K_{r}} \mathrm{D}_{\mathrm{x}, \mathrm{t}}\left[\begin{array}{l}
\zeta \\
\zeta
\end{array}\right] \cdot\left[\begin{array}{l}
1 \\
1
\end{array}\right]=c k\left(1-p_{r}\right)+h_{r}, \\
{\left[\mathrm{~B}_{1}\right]_{21} } & =\int_{\partial_{\mathrm{o}} K_{r} \cup \partial_{\mathrm{b}}^{+, 1} K_{r}} \mathrm{D}_{\mathrm{x}, \mathrm{t}}\left[\begin{array}{l}
\zeta \\
\zeta
\end{array}\right] \cdot\left[\begin{array}{l}
\zeta \\
0
\end{array}\right]=-\frac{c k p_{r}-c k-h_{r}}{3}, \\
{\left[\mathrm{~B}_{1}\right]_{31} } & =\int_{\partial_{\mathrm{o}} K_{r} \cup \partial_{\mathrm{b}}^{+, 1} K_{r}} \mathrm{D}_{\mathrm{x}, \mathrm{t}}\left[\begin{array}{l}
\zeta \\
\zeta
\end{array}\right] \cdot\left[\begin{array}{l}
0 \\
\zeta
\end{array}\right]=-\frac{c k p_{r}-c k-h_{r}}{3} .
\end{aligned}
$$

Thus

$$
\mathrm{B}=\left[\begin{array}{ccc}
0 & 0 & c k\left(1-p_{r}\right)+h_{r} \\
-\frac{h_{r}}{2} & \frac{c k\left(p_{r}-1\right)}{2} & -\frac{c k\left(p_{r}-1\right)-h_{r}}{3} \\
\frac{c k\left(p_{r}-1\right)}{2} & -\frac{h_{r}}{2} & -\frac{c k\left(p_{r}-1\right)-h_{r}}{3}
\end{array}\right]
$$


and its inverse is given by

$$
\mathrm{B}^{-1}=\left[\begin{array}{ccc}
\frac{2}{3\left(c k\left(p_{r}-1\right)-h_{r}\right)} & \frac{2 h_{r}}{\left(h_{r}-c k\left(1-p_{r}\right)\left(h_{r}+c k\left(1-p_{r}\right)\right)\right.} & \frac{-2 c k\left(p_{r}-1\right)}{\left(h_{r}-c k\left(1-p_{r}\right)\right)\left(h_{r}+c k\left(1-p_{r}\right)\right)} \\
\frac{-2}{3\left(c k\left(p_{r}-1\right)-h_{r}\right)} & \frac{-2 c k\left(p_{r}-1\right)}{\left(h_{r}-c k\left(1-p_{r}\right)\right)\left(h_{r}+c k\left(1-p_{r}\right)\right)} & \frac{2 h_{r}}{\left(h_{r}-c k\left(1-p_{r}\right)\right)\left(h_{r}+c k\left(1-p_{r}\right)\right)} \\
\frac{-1}{c k\left(p_{r}-1\right)-h_{r}} & 0 & 0
\end{array}\right] .
$$

Since we are only interested in the last component of $\mathrm{B}^{-1}\left[\begin{array}{l}F_{1} \\ F_{2} \\ F_{3}\end{array}\right]$, which gives the value of $U_{t}=V_{t}$, we only need to calculate

$$
\begin{aligned}
F_{1} & =-\int_{\partial K} \mathrm{D}_{\mathrm{x}, \mathrm{t}}\left(\left[\begin{array}{l}
U_{b} \\
V_{b}
\end{array}\right] \zeta_{b}+\left[\begin{array}{l}
U_{r} \\
V_{r}
\end{array}\right] \zeta_{r}\right) \cdot\left[\begin{array}{l}
1 \\
1
\end{array}\right] \\
& =-\left(U_{b}+V_{b}\right) \int_{\partial K}\left(n_{t}-c n_{x}\right) \zeta_{b}-\left(U_{r}+V_{r}\right) \int_{\partial K}\left(n_{t}-c n_{x}\right) \zeta_{r} .
\end{aligned}
$$

Thus, computing

$$
\int_{\partial_{\mathrm{i}} K} n_{t} \zeta_{b}, \int_{\partial_{\mathrm{i}} K \cup \partial_{\mathrm{b}}^{+, 1}} n_{x} \zeta_{b}, d t, \int_{\partial K_{r}} n_{t} \zeta_{r}, \text { and } \int_{\partial K_{r}} n_{x} \zeta_{r},
$$

we obtain that

$$
U_{t}=V_{t}=\frac{1}{c k\left(1-p_{k}\right)+h_{r}} F_{1}
$$

Rewriting this formula we obtain (3.4.12). Similar calculations are done to obtain the result for tents of Type III.

3.4.4. Error analysis on uniform grids. We now work out the stencil given by the method on a uniform grid where all tents are shaped the same (see Figure 3.6). The stencil translates 3.4 .12 into an equation that gives the nodal values of the outflow apex vertex, given the nodal values at the inflow vertices. Let $h>0$ be the uniform spatial mesh size, $k>0$ be the time step size measured, as before, by the height of the tent pole. At a point $(j h / 2, k n / 2)$ in the lattice $(h / 2) \mathbb{Z} \times(k / 2) \mathbb{Z}$, let $\left(U_{j}^{n}, V_{j}^{n}\right)$ denote the nodal value of the approximation to $z_{h}$ there. As shown in 


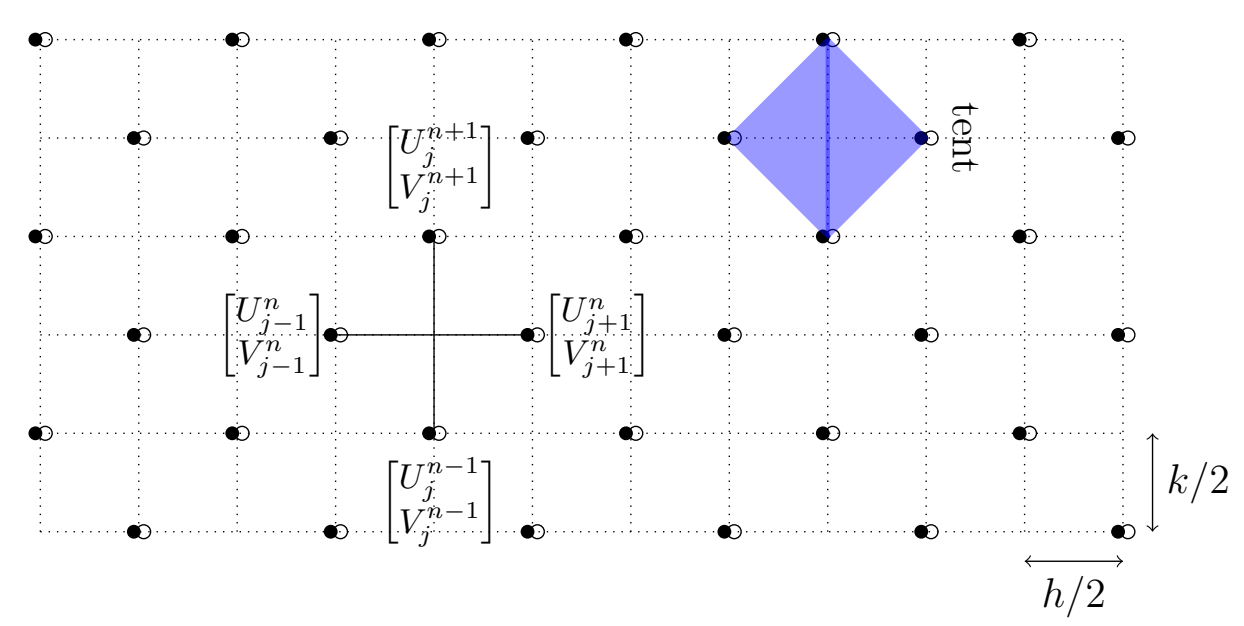

Figure 3.6. The stencil

Figure 3.6, the scheme uses only a subset of lattice points in $h \mathbb{Z} \times k \mathbb{Z}$. Each grid point involved in the scheme has an associated $U$ value (indicated in the figure by "•") and a $V$ value (indicated by "o").

Equation 3.4.12 now simplifies to

$$
\begin{aligned}
& U_{j}^{n+1}=U_{j}^{n-1}+a c\left(V_{j+1}^{n}-V_{j-1}^{n}\right) \\
& V_{j}^{n+1}=V_{j}^{n-1}+a c\left(U_{j+1}^{n}-U_{j-1}^{n}\right)
\end{aligned}
$$

where $a=k / h$. This is simply the non-staggered leapfrog scheme (studied extensively for scalar equations) applied to the first order system. By a simple Taylor expansion about the stencil center, we see that the scheme is consistent and that the local truncation error is of second order (see [57, 65] for definitions of these and related terminology). 
To examine stability, introduce a new vector variable $X_{j}^{n}$ and rewrite the scheme 3.4.17) as follows:

$$
X_{j}^{n+1}=\left[\begin{array}{c}
{\left[X_{j}^{n}\right]_{3}+a c\left[X_{j+1}^{n}-X_{j-1}^{n}\right]_{2}} \\
{\left[X_{j}^{n}\right]_{4}+a c\left[X_{j+1}^{n}-X_{j-1}^{n}\right]_{1}} \\
{\left[X_{j}^{n}\right]_{1}} \\
{\left[X_{j}^{n}\right]_{2}}
\end{array}\right], \quad \text { where }\left[\begin{array}{c}
U_{j}^{n} \\
V_{j}^{n} \\
U_{j}^{n-1} \\
V_{j}^{n-1}
\end{array}\right] .
$$

Thus,

$$
\left[X_{j}^{n+1}\right]=A_{-1}\left[X_{j}^{n}\right]+A_{0}\left[X_{j}^{n}\right]+A_{1}\left[X_{j+1}^{n}\right]
$$

with

$$
A_{0}=\left[\begin{array}{llll}
0 & 0 & 1 & 0 \\
0 & 0 & 0 & 1 \\
1 & 0 & 0 & 0 \\
0 & 1 & 0 & 0
\end{array}\right], \quad A_{1}=\left[\begin{array}{cccc}
0 & a c & 0 & 0 \\
a c & 0 & 0 & 0 \\
0 & 0 & 0 & 0 \\
0 & 0 & 0 & 0
\end{array}\right] \text {, and } A_{-1}=A_{1} .
$$

To this one-step scheme, we now apply von Neumann analysis [57, 65].

Letting $X^{n}=\left\{X_{j}^{n}\right\}$ be the vector-valued grid function, then we can write

$$
X^{n+1}=A * X^{n}
$$

Applying Fourier transform, we have $\widehat{X^{n+1}}(s):=\hat{A}(s) \widehat{X^{n}}(s)$. All of these transforms are defined componentwise. Thus, the amplification matrix $\hat{A}(s)$ or $G(s)$, connecting $X_{j}^{n+1}$ to $X_{j}^{n}$ is given by

$$
G=\left[\begin{array}{cccc}
0 & 2 \hat{\imath} & 1 & 0 \\
2 \hat{\imath} s & 0 & 0 & 1 \\
1 & 0 & 0 & 0 \\
0 & 1 & 0 & 0
\end{array}\right]
$$


where $\hat{\imath}$ denotes the imaginary unit and $s=a c \sin (\theta)$. We write $G=R \Lambda R^{-1}$, by setting

$$
R=\left[\begin{array}{cccc}
1 & 1 & 1 & 1 \\
1 & 1 & 1 & 1 \\
g_{1}^{-1} & g_{2}^{-1} & g_{3}^{-1} & g_{4}^{-1} \\
g_{1}^{-1} & g_{2}^{-1} & -g_{3}^{-1} & -g_{4}^{-1}
\end{array}\right], \quad \Lambda=\left[\begin{array}{cccc}
g_{1} & 0 & 0 & 0 \\
0 & g_{2} & 0 & 0 \\
0 & 0 & g_{3} & 0 \\
0 & 0 & 0 & g_{4}
\end{array}\right],
$$

the eigenvalues of $G$ are $g_{1}=\hat{\imath} s-\sqrt{1-s^{2}}, g_{2}=\hat{\imath} s+\sqrt{1-s^{2}}, g_{3}=-\hat{\imath} s-\sqrt{1-s^{2}}, g_{4}=$ $-\hat{\imath} s+\sqrt{1-s^{2}}$ and $\theta \in[-\pi, \pi]$ gives the frequency in von Neumann analysis. If

$$
|a c|<1
$$

then all eigenvalues satisfy $\left|g_{i}\right|=1$. Furthermore, since

$$
\begin{aligned}
\operatorname{det} R & =4\left(g_{1}^{-1}-g_{2}^{-1}\right)\left(g_{4}^{-1}-g_{3}^{-1}\right) \\
& =-8 \sqrt{1-s^{2}}
\end{aligned}
$$

remains away from zero whenever 3.4.18 holds, the powers $G^{n}=R \Lambda^{n} R^{-1}$ are uniformly bounded for all $n$ and all $\theta$. Hence (3.4.18) implies that the scheme is stable.

We thus conclude, by the Lax-Richtmyer theorem, that the scheme is convergent and is of second order. Note that the causality condition we previously found on general meshes, namely 3.3.3), when restricted to uniform meshes, gives exactly the same CFL condition (3.4.18) obtained above from von Neumann analysis.

\subsection{Numerical results}

3.5.1. Convergence study. First, we report numerical results from our tent pitching (TP) scheme and compare it with the well-known "central-time centralspace" (CTCS) finite difference scheme (see [14, 57], sometimes also known as the 


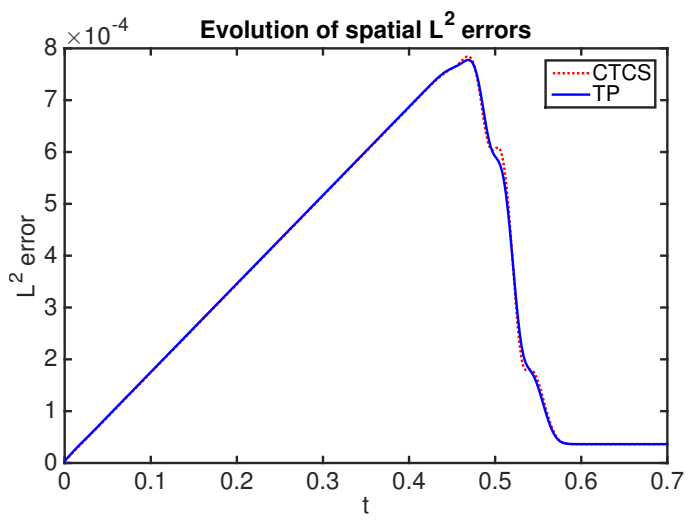

(a) Errors as a function of time

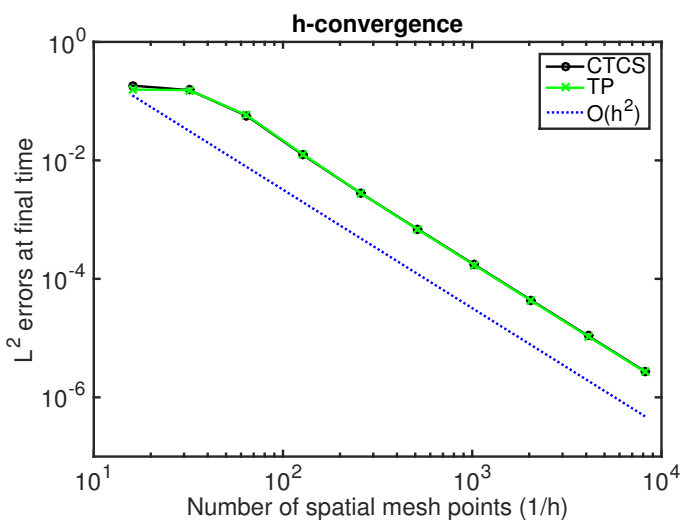

(b) Second order convergence

Figure 3.7. Comparison with CTCS scheme

Yee scheme [41, 70]). The only difference between the two is that while the TP scheme sets the $U$ and $V$ nodes on the same location (exactly as indicated in Figure 3.6), the CTCS scheme sets them on staggered locations on the same grid. Both schemes are applied to the model problem (3.4.1) on uniform grids with $S=1$. We use a grid like that in Figure 3.6 for both methods.

To impose the outgoing impedance boundary conditions within the CTCS scheme, we use the standard finite difference technique of introducing ghost points to the left and right of the finite grid and eliminating the unknown values at those points using the boundary condition. In contrast, in the TP scheme, the impedance boundary conditions are essentially imposed within the finite element spaces, as we have already seen previously. We set $c=1$ and impose the initial condition so that the exact solution is

$$
u_{1}(x, t)=u_{2}(x, t)=e^{-1000((x+t)-1 / 2)^{2}},
$$

i.e., the solution is a smooth pulse moving to the left at unit speed, eventually clearing out of the simulation domain. At every other time step (in the uniform spacetime grid) we compute the $L^{2}(0,1)$-norm of the difference between the computed and exact 
solution. The evolution of these errors in time on a grid of spatial mesh size $h=0.0025$ and $k=0.9 h$ is shown in Figure 3.7 a.

We observe from Figure 3.7a that the errors of both methods are comparable and remain low throughout the simulated time. Note also that after the pulse clears the simulation domain reflectionlessly (and the solution within $[0,1]$ vanishes), the errors for both methods decrease markedly. In Figure 3.7, we display a log-log plot of the $L^{2}(0,1)$-norm of the errors at $t=0.5$ for $h=1 / 2^{3}, \ldots, 1 / 2^{13}$, and $k=0.9 h$. The rate of decrease of this error is clearly seen to be of the order $O\left(h^{2}\right)$. This is in accordance with our von Neumann analysis of $\S 3.4 .4$ (although we did not take into account boundary conditions in that analysis).

Thus we conclude from Figure 3.7 that there is negligible difference between the performance of the two methods on uniform grids.

3.5.2. Material interfaces and other boundary conditions. Next, we consider a generalization of 3.4 .1 given by

$$
\begin{array}{rlrl}
\partial_{t}\left[\begin{array}{ll}
\kappa_{1} & 0 \\
0 & \kappa_{2}
\end{array}\right]\left[\begin{array}{l}
u_{1} \\
u_{2}
\end{array}\right]-\left[\begin{array}{ll}
0 & c \\
c & 0
\end{array}\right] \partial_{x}\left[\begin{array}{l}
u_{1} \\
u_{2}
\end{array}\right] & =f, & & 0<x<1,0<t<T, \\
u_{1}(x, 0) & =u_{1}^{0}(x), & & 0<x<1, \\
u_{2}(x, 0) & =u_{2}^{0}(x), & 0<x<1, \\
z_{0} u_{1}-u_{2} & =0, & & x=0,0<t<T, \\
z_{1} u_{1}+u_{2} & =0, & & x=1,0<t<T .
\end{array}
$$

where $\kappa_{1}(x)$ and $\kappa_{2}(x)$ are time-independent material parameters and $c, z_{0}$ and $z_{1}$ are constants. Such systems arise from electromagnetics or acoustics [41, 47] on layered media and the differential equation is often written in the following equivalent, but 
non-symmetric form

$$
\partial_{t}\left[\begin{array}{l}
u_{1} \\
u_{2}
\end{array}\right]-\left[\begin{array}{cc}
0 & \beta_{1} \\
\beta_{2} & 0
\end{array}\right] \partial_{x}\left[\begin{array}{l}
u_{1} \\
u_{2}
\end{array}\right]=\tilde{f}
$$

where $\beta_{i}(x)=c / \kappa_{i}(x)$ and $\tilde{f}=\operatorname{diag}\left(\kappa_{1}^{-1}, \kappa_{2}^{-1}\right) f$ obtained by scaling the equations of (3.5.1a) by $\kappa_{1}^{-1}$ and $\kappa_{2}^{-1}$. When $\kappa_{1}(x) \equiv \kappa_{2}(x) \equiv 1$ and $z_{0}=z_{1}=1$, we obtain the model formulation we discussed previously in detail. Dirichlet boundary conditions can be imposed by putting $z_{0}=z_{1}=0$, while exact outgoing impedance conditions can be imposed using $z_{0}=\sqrt{\kappa_{1} / \kappa_{2}}$ and $z_{1}=\sqrt{\kappa_{1} / \kappa_{2}}$. Intermediate values of $z_{i}$ give damped impedance boundary conditions.

Whenever $\kappa_{i}$ is a constant on each spatial mesh interval, a tent pitching scheme is suggested by a simple generalization of the previous algorithm for homogeneous media. We define the discrete spaces exactly as in 3.4 .9 , but noting that $V(\Omega)$ now has different essential boundary conditions - stemming from (3.5.1d)-(3.5.1e which are inherited by the spaces on tents with its tent pole on the boundary. The generalization of the scheme is derived by merely setting the $A$ and $\mathrm{D}_{\mathrm{x}, \mathrm{t}}$ in 3.4.11 by

$$
A=\left[\begin{array}{cc}
\kappa_{1} & 0 \\
0 & \kappa_{2}
\end{array}\right] \partial_{t}-\left[\begin{array}{ll}
0 & c \\
c & 0
\end{array}\right] \partial_{x}, \quad \mathrm{D}_{\mathrm{x}, \mathrm{t}}=\left[\begin{array}{cc}
n_{t} \kappa_{1} & -c n_{x} \\
-c n_{x} & n_{t} \kappa_{2}
\end{array}\right]
$$

Note that this $A$, appearing on the left hand side of (3.5.1a), satisfies A-a, A-b and $\mathrm{A}-\mathrm{c}$. By solving this general version of (3.4.11 one can obtain propagation formulas similar to 3.4 .12 , but we omit these details and report only the numerical results.

First we consider the case

$$
\kappa_{1}=\left\{\begin{array}{ll}
2, & 0<x<1 / 2, \\
1, & 1 / 2<x<1,
\end{array} \quad \kappa_{2}= \begin{cases}2, & 0<x<1 / 2, \\
1, & 1 / 2<x<1,\end{cases}\right.
$$



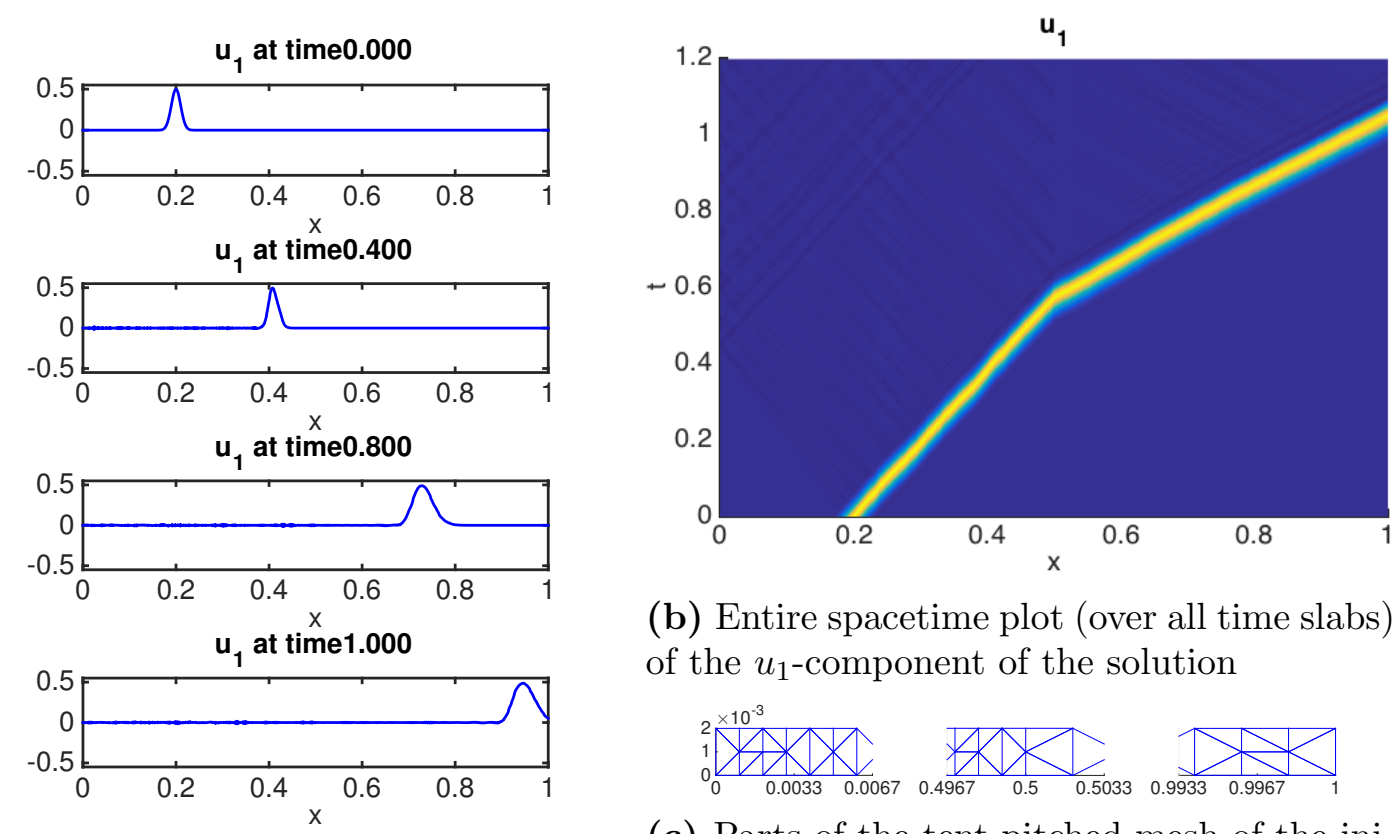

(b) Entire spacetime plot (over all time slabs) of the $u_{1}$-component of the solution

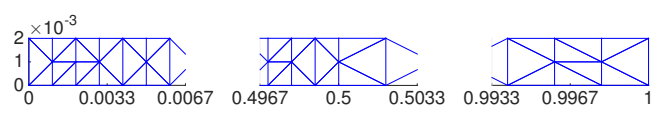

(c) Parts of the tent pitched mesh of the ini-

(a) Time snapshots of $u_{1}$ (cf. whole spacetime plot of $u_{1}$ in Figure $3.8 \mathrm{~b}$

tial time slab, with varying spatial mesh sizes in the regions $x<0.5$ and $x>0.5$.

Figure 3.8. Wave propagation through an impedance-matched interface

and $c=1$. The wave speed (equaling $c / \sqrt{\kappa_{1} \kappa_{2}}$ ), jumps from 0.5 in the left half to 1 in the right half. However, the impedance (equaling $\kappa_{1} / \kappa_{2}-$ see [47]) is one in both regions. Thus $x=0.5$ is an impedance-matched interface about which we do not expect to see any reflection.

We use the tent pitching method to simulate a wave propagating to the right starting near $x=0.2$. To this end, define a smooth pulse $g(x)=e^{-5000(x-0.2)^{2}}$ and set the data in 3.5.1 by

$$
f=0, \quad u_{1}^{0}(x)=\left(c / \kappa_{1}\right) g(x), \quad u_{2}^{0}(x)=-\left(c / \sqrt{\kappa_{1} \kappa_{2}}\right) g(x),
$$

and $z_{0}=\sqrt{\kappa_{1} / \kappa_{2}}$ and $z_{1}=\sqrt{\kappa_{1} / \kappa_{2}}$. We use a spatial mesh of mesh size $h=10^{-3}$ in the left half and $h=2 \times 10^{-3}$ in the right half. A simple tent meshing algorithm then produces a mesh of spacetime tents based on this non-uniform spatial mesh that 
satisfies the CFL condition 3.3.3). The meshing algorithm proceeds as illustrated as in Figure 3.1 by simply picking a point with the lowest time coordinate to pitch a tent. When multiple locations have the minimal time coordinate, the algorithm picks a tent pitching location among them randomly, thus giving an unstructured mesh. To minimize the overhead in constructing the mesh of tents, instead of meshing the entire spacetime domain at once, we first mesh a thin time slab $\{(x, t): 0<t<$ $0.002,0<x<1\}$ and then repeatedly stack this mesh in time to cover the entire region of time simulation. The mesh of the initial slab is shown in Figure $3.8 \mathrm{c}$.

One of the two components of the computed solution is shown in the remaining two plots of Figure 3.8. Clearly, the simulated wave packet travels left across the $x=0.5$ interface without any reflected wave and expands as it enters the region of higher wave speed. In further (unreported) numerical experiments, we have noticed changes in the discrete wave speed depending on the spacetime mesh. For example, the wave speed differs if one uses uniform space time meshes with positively sloped diagonals only or negatively sloped diagonals only. Such wave speed differences appear to approach to zero slowly as $h$ is made smaller. High order methods may be needed to reduce these dispersive errors.

Our next and final example involves an interface where we expect both reflection and transmission. We set $c=1$ and

$$
\kappa_{1}=\left\{\begin{array}{cl}
4, & 0<x<1 / 2, \\
1 / 2, & 1 / 2<x<1,
\end{array} \quad \kappa_{2}=\left\{\begin{array}{cc}
1, & 0<x<1 / 2 \\
1 / 2, & 1 / 2<x<1
\end{array}\right.\right.
$$

Both the wave speed and the impedance jumps from the left region to the right region (from 0.5 and 4 to 2 and 1, respectively). We set $f$ and initial data as in the last simulation by (3.5.2), but in order to impose Dirichlet boundary condition, we set $z_{0}=z_{1}=0$. This time, instead of using a non-uniform mesh, we use a spatially 


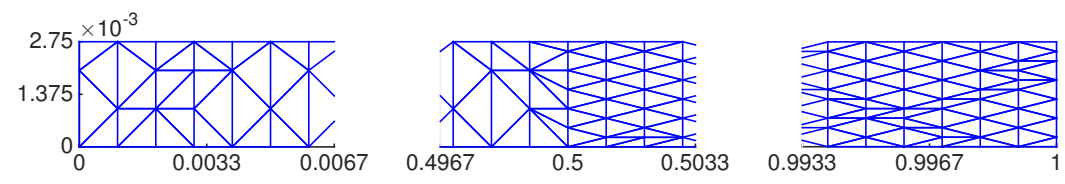

(a) Left, middle and right parts of the tent pitched mesh on one time slab

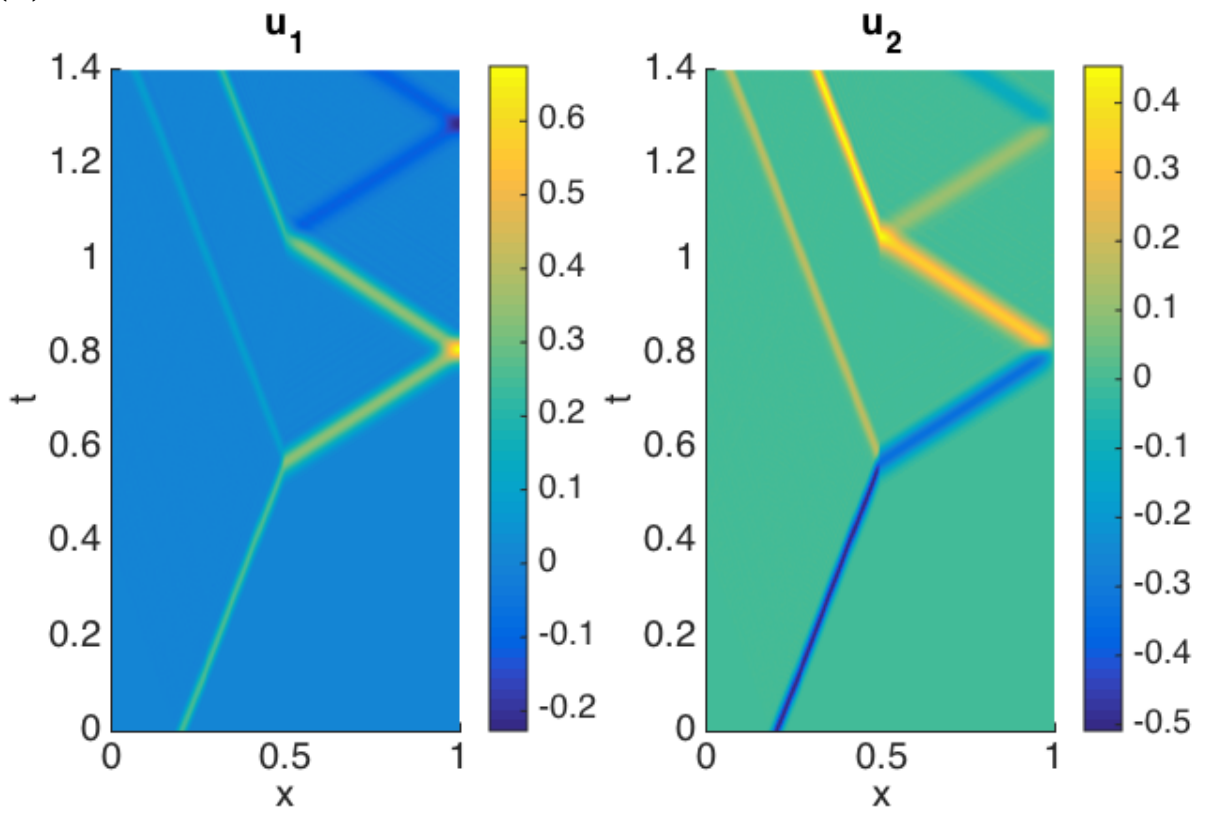

(b) Spacetime plot of the two solution components computed by explicit tent pitching

Figure 3.9. Case of reflected and transmitted waves

uniform mesh of $h=10^{-3}$ and let the tent pitching algorithm adjust $k$ to satisfy the CFL condition (3.3.3) in each tent. We found that the mesh obtained, displayed in Figure 3.9a, while not ideal due to the thin triangles, is adequate for the simulation. (Better tent pitched meshes can be obtained using non-uniform spatial mesh spacing, as we saw in the previous example and Figure 3.8c.) The solution components $u_{1}$ and $u_{2}$ obtained from the simulation are displayed in Figure $3.9 \mathrm{~b}$. The computed waves are transmitted as well as reflected both from the interface and the Dirichlet boundaries. The expected features of the solution are therefore recovered by the method. 


\section{A spacetime DPG method for the Schrodinger equation}

\subsection{Introduction to the problem}

This chapter is devoted to exploring a weak formulation and an accompanying numerical technique for the Schrödinger equation with Dirichlet boundary conditions. Let $\Omega_{0} \subset \mathbb{R}^{d-1}(d>1)$ be an open bounded domain with Lipschitz boundary. The space variable $x$ lies in $\Omega_{0}$ while the time variable $t$ lies in the open interval $(0, T)$ with $T<\infty$. The classical form of Schrödinger initial boundary value problem reads as follows:

$$
\begin{aligned}
\hat{\imath} \partial_{t} u-\Delta_{x} u & =f, & & x \in \Omega_{0}, 0<t<T, \\
u(x, t) & =0, & & x \in \partial \Omega_{0}, 0<t<T, \\
u(x, 0) & =0, & & x \in \Omega_{0},
\end{aligned}
$$

where $\partial_{t}$ denotes the time derivative and $\Delta_{x}$ denotes the Laplacian with respect to the spatial variable $x$ and $\hat{\imath}$ is the imaginary unit. Here $f$ is any given function in $L^{2}(\Omega)$ and $\Omega=\Omega_{0} \times(0, T)$ throughout.

Many applications of interest come as a first order system even if they are often displayed as second order partial differential equations. For example, the second order heat equation is really a combination of two first order equations, namely the Fourier law of heat conduction and the conservation of energy. Similarly, the linear elasticity equation while often displayed as a second order equation for displacement, is really a combination of the two first order equations, the constitutive (Hooke's) law 
and the equation of static equilibrium. Thus its no surprise that it makes physical sense to bring back the heat equation or the elasticity equation to first order form before discretizing. However, it makes no physical sense to do this for the Schrödinger equation as its not derived from first order physical laws.

It makes no mathematical sense either. One might be tempted to introduce a "flux" $\tau$, formulate the first order system $\hat{\imath} \partial_{t} u-\operatorname{div}_{x} \tau=f$ and $\nabla_{x} u-\tau=g$, and claim its equivalence to 4.1.1) when $g=0$. This claim is false because while the Schrödinger problem 4.1.1) is wellposed for $f \in L^{2}(\Omega)$, the first order system cannot be wellposed in $L^{2}(\Omega)$. Indeed, denoting the norm of $L^{2}(\Omega)$ by $\|\cdot\|_{\Omega}$, if the first order system were wellposed, then there are constants $C_{1}, C_{2}>0$ such that $\|u\|_{\Omega}+\|\tau\|_{\Omega} \leq C_{1}\|f\|_{\Omega}+C_{2}\|g\|_{\Omega}$. But then the second equation of the system implies that $\left\|\nabla_{x} u\right\|_{\Omega}=\|g+\tau\|_{\Omega} \leq C_{1}\|f\|_{\Omega}+2 C_{2}\|g\|_{\Omega}$ for any solution $u$, which is false: In the next two paragraphs we will exhibit a Schrödinger solution for which $\left\|\nabla_{x} u\right\|_{\Omega}=\infty$ even when $g=0$ and $f \in L^{2}(\Omega)$.

First observe that given any $f(x, t)$ in $L^{2}(\Omega)$, it is possible to solve (4.1.1) by the "method of Galerkin approximations" [32] (distinct from the Galerkin finite element method). Let $e_{k}(x)$ in $H_{0}^{1}\left(\Omega_{0}\right)$ and $\omega_{k}^{2}>0$ be an eigenpair of $\Delta_{x}$ satisfying

$$
-\Delta_{x} e_{k}=\omega_{k}^{2} e_{k} \quad \text { a.e. in } \Omega_{0}
$$

normalized so that $\left\|e_{k}\right\|_{\Omega_{0}}=1$ for all natural numbers $k \geq 1$. Since Fubini's theorem for product measures implies that $f(\cdot, t)$ is in $L^{2}\left(\Omega_{0}\right)$, the following definitions make sense:

$$
\begin{aligned}
f_{k}(t) & =\int_{\Omega_{0}} f(x, t) \bar{e}_{k}(x) d x, & u_{k}(t) & =-\hat{\imath} \int_{0}^{t} e^{\hat{\imath} \omega_{k}^{2}(t-s)} f_{k}(s) d s, \\
F_{M}(x, t) & =\sum_{k=1}^{M} f_{k}(t) e_{k}(x), & U_{M}(x, t) & =\sum_{k=1}^{M} u_{k}(t) e_{k}(x) .
\end{aligned}
$$




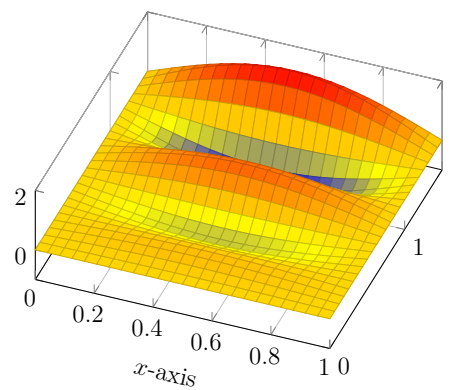

(a) $M=1$

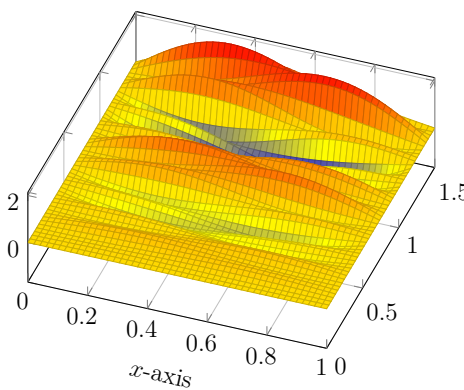

(b) $M=2$

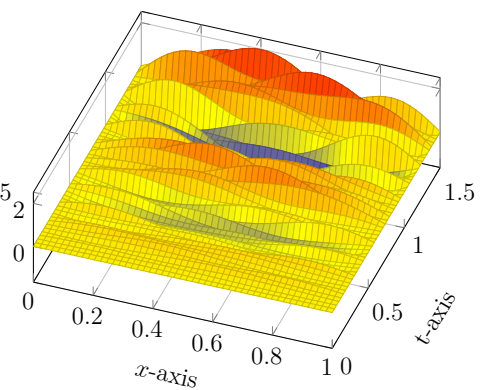

(c) $M=4$

Figure 4.1. Real part of $U_{M}(x, t)$ with different values of $M$.

It is not difficult to show (see the proof of Theorem 4.2 .3 below) that $u=\lim _{M \rightarrow \infty} U_{M}$ exists in $L^{2}(\Omega)$ and solves 4.1.1.

Now consider the one-dimensional case $\Omega_{0}=(0,1)$ where $\omega_{k}=k \pi$ and choose

$$
f(x, t)=\sum_{k=1}^{\infty} \frac{1}{k} e^{\hat{i} \omega_{k}^{2} t} e_{k}(x) \quad \text { in } L^{2}(\Omega) .
$$

Then by the orthonormality of $e_{k}$, we have that $f_{k}(s)=e^{\hat{\imath} \omega_{k}^{2} s} / k, u_{k}(t)=-i t e^{\hat{\imath} \omega_{k}^{2} t} / k$,

$$
\begin{aligned}
\left\|U_{M}\right\|_{\Omega}^{2} & =\sum_{k=1}^{M} \int_{0}^{T}\left|u_{k}(t)\right|^{2} d t=\sum_{k=1}^{M} \int_{0}^{T}\left|\frac{-\hat{\imath} t}{k} e^{\hat{\imath} \omega_{k}^{2} t}\right|^{2} d t=\frac{T^{3}}{3} \sum_{k=1}^{M} \frac{1}{k^{2}} \\
\left\|\nabla_{x} U_{M}\right\|_{\Omega}^{2} & =\sum_{k=1}^{M} \omega_{k}^{2} \int_{0}^{T}\left|u_{k}(t)\right|^{2} d t=T^{3} \sum_{k=1}^{M} \frac{\omega_{k}^{2}}{3 k^{2}}=\frac{\pi^{2}}{3} T^{3} M .
\end{aligned}
$$

The solution $u$ is the limit of $U_{M}$. The above calculations clearly show that as $M \rightarrow$ $\infty$, while $\|u\|_{\Omega}=\lim _{M \rightarrow \infty}\left\|U_{M}\right\|_{\Omega}=\left(T^{3} \pi^{2} / 18\right)^{\frac{1}{2}}$, the limit of $\left\|\nabla_{x} U_{M}\right\|_{\Omega}$ diverges. Thus it is possible to obtain a Schrödinger solution $u$ whose $H^{1}$-norm is infinite even when $f \in L^{2}(\Omega)$. Note that finer arguments are needed to understand the regularity of Schrödinger solutions in unbounded domains, which although a topic of wide mathematical interest [66], is not our concern here.

The second order form necessitates formulations in the non-standard graph spaces of the second order Schrödinger operator. The general spaces and arguments required 
for this analysis are collected in a standalone Chapter 1 anticipating uses outside of the Schrödinger example. Borrowing the approach of [30], we are able to prove wellposedness without developing a trace theory for the graph spaces. Numerical methods using the strong formulation must use conforming finite element subspaces of the graph spaces. On the other hand, numerical methods using the weak formulation need only use existing standard finite element spaces. In either case, an interpolation theory in the Schrödinger graph norm is needed to estimate convergence rates. We address this issue in one space dimension.

In the next section, we investigate wellposedness (in the sense of Hadamard) for a strong and weak variational formulation for the Schrödinger problem. This will require an abstract definition of a boundary operator and duality pairings in a graph space. Such abstract definitions that apply beyond the Schrödinger setting are in Chapter 1 .

We now provide a functional setting within which a strong and a weak formulation of the spacetime Schrödinger problem can be proved to be wellposed (i.e., inf-sup stable). The analysis is an application of the general theory detailed in Chapter 1 .

\subsection{Wellposed formulation}

The classical form of the problem is already presented in 4.1.1. Recalling that $\Omega=\Omega_{0} \times(0, T)$, define these parts of $\partial \Omega$ :

$$
\Gamma=\partial \Omega_{0} \times[0, T] \cup \Omega_{0} \times\{0\}, \quad \Gamma^{*}=\partial \Omega_{0} \times[0, T] \cup \Omega_{0} \times\{T\}
$$

(see Fig. 4.2). Then the initial and boundary conditions together can be written as $\left.u\right|_{\Gamma}=0$. We want to write (4.1.1) as an operator equation (see (1.4.1) ) to apply the 
general results of Chapter 1. To this end, consider the setting of Chapter 1 with

$$
A=A^{*}=\hat{\imath} \partial_{t}-\Delta_{x}, \quad k=l=m=1 .
$$

and moreover, Assumptions $\mathrm{A}-\mathrm{a}$ and $\mathrm{A}-\mathrm{b}$ are satisfied for these operators. The space $W=W^{*}$ is then defined by $\left.1.2 .1-1.2 .2\right) ;$ namely $W=W^{*}=\left\{u \in L^{2}(\Omega)\right.$ : $\left.\hat{\imath} \partial_{t} u-\Delta_{x} u \in L^{2}(\Omega)\right\}$. The operator $D=D^{*}: W \rightarrow W^{\prime}$ is defined by 1.2.3 1.2 .4 ; namely $\langle D w, \tilde{w}\rangle_{W}=(A w, \tilde{w})_{\Omega}-(w, A \tilde{w})_{\Omega}$ for all $w, \tilde{w} \in W$. As usual, let $\mathcal{D}(\bar{\Omega})$ denote the restrictions of functions from $\mathcal{D}\left(\mathbb{R}^{n+1}\right)$ to $\Omega$. The operator $D$ (often called the "boundary operator" in the theory of Friedrichs systems [30]), satisfies

$$
\langle D \phi, \psi\rangle_{W}=\int_{\partial \Omega} \hat{\imath} n_{t} \phi \bar{\psi}+\int_{\partial \Omega} \phi\left(n_{x} \cdot \nabla_{x} \bar{\psi}\right)-\int_{\partial \Omega}\left(n_{x} \cdot \nabla_{x} \phi\right) \bar{\psi}
$$

for all $\phi, \psi \in \mathcal{D}(\bar{\Omega})$. Note that although the integrals on the right hand side need not exist for all functions in $W, D$ is defined on all $W$ through 1.2.3.

Although we set the differential operators $A$ and $A^{*}$ to be equal above, note that we consider each as an unbounded operator with its own domain. We set the domain of $A$ to

$$
\operatorname{dom}(A)=\left\{u \in W:\langle D v, u\rangle_{W}=0, \forall v \in \mathcal{V}^{*}\right\}
$$

where $\mathcal{V}^{*}=\left\{\varphi \in \mathcal{D}(\bar{\Omega}):\left.\varphi\right|_{\Gamma^{*}}=0\right\}$. Since $\mathcal{D}(\Omega) \subset \operatorname{dom}(A)$, then Assumption A-c holds. An therefore there exists a unique The domain of the adjoint operator is given as in (1.3.1), and satisfy . Therefore is a by $\operatorname{dom}\left(A^{*}\right)=\left\{s \in L^{2}(\Omega)\right.$ : there is an $\ell \in L^{2}(\Omega)$ such that $(A v, s)_{\Omega}=(v, \ell)_{\Omega}$ for all $\left.v \in \operatorname{dom}(A)\right\}$. Here $(\cdot, \cdot)_{\Omega}$ denotes the (complex) inner product in $L^{2}(\Omega)$. Finally, as in Chapter 1 , set $V=\operatorname{dom}(A)$ and $V^{*}=\operatorname{dom}\left(A^{*}\right)$ with the understanding that both $V$ and $V^{*}$ are endowed with the $W$-topology, while $\operatorname{dom}(A)$ and $\operatorname{dom}\left(A^{*}\right)$ have the topology of $L^{2}(\Omega)$. 

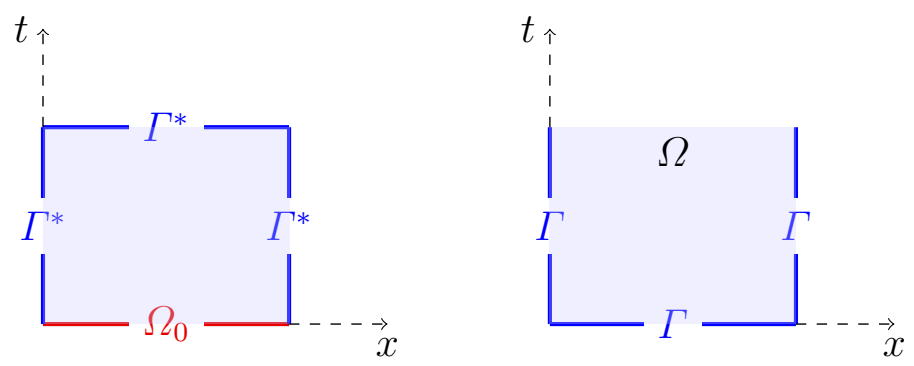

Figure 4.2. Schematic of the spacetime domain

Since conditions (A-a), A-b and (A-c) are easily verified using 4.2.1). Hence Lemma 1.3.1 shows that $\operatorname{dom}\left(A^{*}\right)$ equals

$$
V^{*}={ }^{\perp} D(V) .
$$

Rewriting 4.2.2 in the same style,

$$
V={ }^{\perp} D\left(\mathcal{V}^{*}\right)
$$

Thus $V$ and $V^{*}$ are closed subspaces of $W$. Let $\mathcal{V}=\left\{\varphi \in \mathcal{D}(\bar{\Omega}):\left.\varphi\right|_{\Gamma}=0\right\}$.

$$
\text { LEMMA 4.2.1. } \mathcal{V} \subset V \text { and } \mathcal{V}^{*} \subset V^{*}
$$

Proof. Equation (4.2.1) implies $\left\langle D \phi, \phi^{*}\right\rangle_{W}=0$ for all $\phi \in V$ and $\phi^{*} \in \mathcal{V}^{*}$. Hence, any $\phi \in \mathcal{V}$ is also in ${ }^{\perp} D\left(\mathcal{V}^{*}\right)$, which by (4.2.4) implies that $\phi \in V$. Thus $\mathcal{V} \subset V$

If $\phi^{*} \in \mathcal{V}^{*}$, then 4.2 .2 shows that $\left\langle D \phi^{*}, u\right\rangle_{W}=0$ for all $u \in V$, i.e., $\phi^{*} \in{ }^{\perp} D(V)$, which by 4.2 .3 implies $\phi^{*}$ is in $V^{*}$.

In 4.2.4, $\mathcal{V}^{*}$ may be replaced by $V^{*}$ provided a density result is available, as it is shown in Lemma 1 . 
4.2.1. Strong formulation. The strong formulation of the Schrödinger problem (4.1.1) is based on these sesquilinear and conjugate linear forms:

$$
a(u, v)=(A u, v)_{\Omega}, \quad l(v)=(f, v)_{\Omega} .
$$

Problem 4.2.2 (Strong formulation). Given any $f \in L^{2}(\Omega)$, find $u \in V$ satisfying

$$
a(u, v)=l(v), \quad \forall v \in L^{2}(\Omega) .
$$

Theorem 4.2.3. Suppose Assumption 1 holds. Then the linear Schrödinger operator $A: V \rightarrow L^{2}(\Omega)$ is a continuous bijection. Hence Problem 4.2.2 is well posed.

ProOf. To prove the surjectivity of $A$, suppose $f \in L^{2}(\Omega)$. Recall the definitions of $e_{k}, u_{k}$ and $f_{k}$ from (4.1.2) and 4.1.3). Clearly, $A U_{M}=F_{M}$. Since $U_{M}$ and any $\varphi \in \mathcal{V}^{*}$ are smooth enough for integration by parts using $\left.\varphi\right|_{\Gamma^{*}}=0$ and $\left.U_{M}\right|_{\Gamma}=0$, we have

$$
\begin{aligned}
\left(\hat{\imath} \partial_{t} U_{M}, \varphi\right)_{\Omega} & =\left(U_{M}, \hat{\imath} \partial_{t} \varphi\right)_{\Omega} \\
\left(\Delta U_{M}, \varphi\right)_{\Omega} & =\left(U_{M}, \Delta \varphi\right)_{\Omega} .
\end{aligned}
$$

Hence $\left\langle D \varphi, U_{M}\right\rangle_{W}=\left(A \varphi, U_{M}\right)_{\Omega}-\left(\varphi, A U_{M}\right)_{\Omega}=0$ for all $\varphi \in \mathcal{V}^{*}$. By 4.2.4), this implies that $U_{M}$ is in $V$.

Next, we show that $U_{M}$ is a Cauchy sequence in $V$. For any $N>M$,

$$
\begin{aligned}
\left\|U_{M}-U_{N}\right\|_{\Omega}^{2} & =\sum_{k=M+1}^{N} \int_{0}^{T}\left|u_{k}(t)\right|^{2} d t \leq \frac{1}{2} T^{2} \sum_{k=M+1}^{\infty} \int_{0}^{T}\left|f_{k}(t)\right|^{2} d t, \\
\left\|A\left(U_{M}-U_{N}\right)\right\|_{\Omega}^{2} & =\left\|F_{M}-F_{N}\right\|_{\Omega}^{2} \leq \sum_{k=M+1}^{\infty} \int_{0}^{T}\left|f_{k}(t)\right|^{2} d t
\end{aligned}
$$

both of which converge to 0 as $M \rightarrow \infty$, because $f \in L^{2}(\Omega)$. Thus $U_{M}$ is Cauchy. It must therefore have an accumulation point $u$ in $V$. Moreover, since $A u$ and $f$ are 
$L^{2}(\Omega)$-limits of the same sequence $F_{M}=A U_{M}$, we have $A u=f$. Thus $A: V \rightarrow$ $L^{2}(\Omega)$ is surjective.

We use a similar argument (with $u_{k}$ defined by integrals from $T$ to $t$ ) to show that $A=A^{*}: V^{*} \rightarrow L^{2}(\Omega)$ is also surjective. We omit the details, but note that the only difference is that instead of (4.2.4), we must now use

$$
V^{*}={ }^{\perp} D(\mathcal{V}),
$$

which follows from 4.2.3), Assumption 1 and Lemma 2.1.2. Finally, since $\operatorname{ker}(A)=$ ${ }^{{ }^{\perp}} \operatorname{ran}\left(A^{*}\right)$, the surjectivity of $A^{*}: V^{*} \rightarrow L^{2}(\Omega)$ shows that $A: V \rightarrow L^{2}(\Omega)$ is injective, thus completing the proof of the stated bijectivity.

REMark 4.2.4. An example of a standard wellposedness result for the Schrödinger equation obtained using semigroup theory is [46, Theorem 4.8.1], which proves that there is one and only one solution to (4.1.1) whenever $f \equiv 0$ and $u(x, 0)$ is in $H^{2}(\Omega) \cap$ $H_{0}^{1}(\Omega)$. In Theorem 4.2.3, we have shown (by a different method) that the existence of a unique solution holds for any $f \in L^{2}(\Omega)$. Note that in the above proof, we used Assumption 1 only to obtain injectivity. If one opts to use the results of [46] (with $u(x, 0) \equiv 0$ and $f \equiv 0$ ) to conclude injectivity, then there is no need to place Assumption 1 in Theorem 4.2.3.

4.2.2. A weak formulation. Now we consider a mesh-dependent weak formulation that is the basis of the DPG method. This formulation, sometimes called the "ultraweak" formulation, is given in a general setting in Problem 1.4 .2 of Chapter 1. We apply this to our example of the Schrödinger equation.

The spacetime domain $\Omega$ is partitioned into a mesh $\Omega_{h}$ of finitely many open elements $K$ such that $\bar{\Omega}=\cup_{K \in \Omega_{h}} \bar{K}$ where $h=\max _{K \in \Omega_{h}} \operatorname{diam}(K)$. Particularizing the general definitions in Chapter 1 (see $(1.4 .2)$ through 1 1.4.4p) to the Schrödinger 
example, we let $A_{h}=A_{h}^{*}$ be the Schrödinger operator applied element by element and let $W_{h}=W_{h}^{*}=\left\{w \in L^{2}(\Omega): A\left(\left.w\right|_{K}\right) \in L^{2}(K)\right.$ for all $\left.K \in \Omega_{h}\right\}$. The operator $D_{h}: W_{h} \rightarrow W_{h}^{\prime}$ is defined by $\left\langle D_{h} w, v\right\rangle_{W_{h}}=\left(A_{h} w, v\right)_{\Omega}-\left(w, A_{h} v\right)_{\Omega}$ for all $w, v \in W_{h}$ and let $D_{h, V}: V \rightarrow W_{h}^{\prime}$ denote $\left.D_{h}\right|_{V}$. The range of $D_{h, V}$, denoted by $Q$, is made into a complete space by the norm $\|q\|_{Q}=\inf _{v \in D_{h, V}^{-1}(\{q\})}\|v\|_{W}$. Abbreviating the duality pairing $\langle\cdot, \cdot\rangle_{W_{h}}$ by $\langle\cdot, \cdot\rangle_{h}$, define the sesquilinear form $b((u, q), v)=\left(u, A_{h} v\right)_{\Omega}+\langle q, v\rangle_{h}$ on $\left(L^{2}(\Omega) \times Q\right) \times W_{h}$.

Problem 4.2.5 (Ultraweak formulation). Given $F \in W_{h}^{\prime}$, find $u \in L^{2}(\Omega)$ and $q \in Q$ such that

$$
b((u, q), v)=F(v), \quad \forall v \in W_{h} .
$$

Theorem 4.2.6. Suppose Assumption 1 holds. Then Problem 4.2.5 is well posed, i.e., there is a $C>0$ such that given any $F \in W_{h}^{\prime}$, there is a unique solution $(u, q) \in$ $L^{2}(\Omega) \times Q$ to Problem 4.2.5 and it satisfies

$$
\|u\|_{\Omega}^{2}+\|q\|_{Q}^{2} \leq C\|F\|_{W_{h}^{\prime}}^{2}
$$

Moreover, if $F(v)=(f, v)_{\Omega}$ for some $f \in L^{2}(\Omega)$, then $u$ is in $V$ and $q=D_{h} u$.

Proof. The result follows from Theorem 1.4.5. Since Lemma 2.1.2 together with (4.2.4 implies (1.4.9) and Theorem 4.2.3 implies 1.4.10, the assumptions of Theorem 1.4.5 are verified.

4.2.3. Verification of the density assumption. In the next three sections, $\Omega_{0}$ is set to be the interval $(0, L)$ and $\Omega=(0, L) \times(0, T)$ where $L, T>0$. The purpose of this section is to verify the density assumption (Assumption 1) in this case of one space dimension. 
Theorem 4.2.7. Let $\Omega=(0, L) \times(0, T)$. Then $\mathcal{V}^{*}=\left\{\varphi \in \mathcal{D}(\bar{\Omega}):\left.\varphi\right|_{\Gamma^{*}}=0\right\}$ is dense in $V^{*}$ and $\mathcal{V}=\left\{\varphi \in \mathcal{D}(\bar{\Omega}):\left.\varphi\right|_{\Gamma}=0\right\}$ is dense in $V$.

Proof. Since the proofs of both the stated density results are similar, we will only show the proof of density of $\mathcal{V}^{*}$ in $V^{*}$.

Step 1. Extend: Let $\Omega_{l}=(-L, 0] \times(0, T)$ and $\Omega_{r}=[L, 2 L) \times(0, T)$. Define an operator $G$ that extends functions on $\Omega$ to $\hat{\Omega} \equiv \Omega_{l} \cup \Omega \cup \Omega_{r}$ by

$$
G w(x, t)= \begin{cases}-w(-x, t), & (x, t) \in \Omega_{l}, \\ -w(2 L-x, t), & (x, t) \in \Omega_{r},\end{cases}
$$

(and $G w(x, t)=w(x, t)$ for all $(x, t) \in \Omega)$. Let $G^{\prime}$ be the reverse operator that maps functions on $\hat{\Omega}$ to $\Omega$ by $G^{\prime} \bar{w}(x, t)=\bar{w}(x, t)-\bar{w}(-x, t)-\bar{w}(2 L-x, t)$, for all $(x, t) \in \Omega$ (see Figure 4.3). Such definitions are to be interpreted a.e., so that for example, $G w$ is well defined for any $w$ in $L^{2}(\Omega)$. It is easy to see by a change of variable that

$$
(G f, g)_{\hat{\Omega}}=\left(f, G^{\prime} g\right)_{\Omega}, \quad \forall f \in L^{2}(\Omega), g \in L^{2}(\hat{\Omega}) .
$$

Next, we claim that

$$
A G v=G A v, \quad \forall v \in V^{*} .
$$

Clearly, $G v$ is in $L^{2}(\hat{\Omega})$. Let $\varphi \in \mathcal{D}(\hat{\Omega})$. Let $\langle A G v, \varphi\rangle_{\mathcal{D}(\hat{\Omega})}$ denote the action of the distribution $A G v$ on $\bar{\varphi}$. Then $\langle A G v, \varphi\rangle_{\mathcal{D}(\hat{\Omega})}=(G v, A \varphi)_{\hat{\Omega}}=\left(v, G^{\prime} A \varphi\right)_{\Omega}$ because of 4.2.5. By the chain rule applied to the smooth function $\varphi$, we find that

$$
G^{\prime} A \varphi=A G^{\prime} \varphi
$$




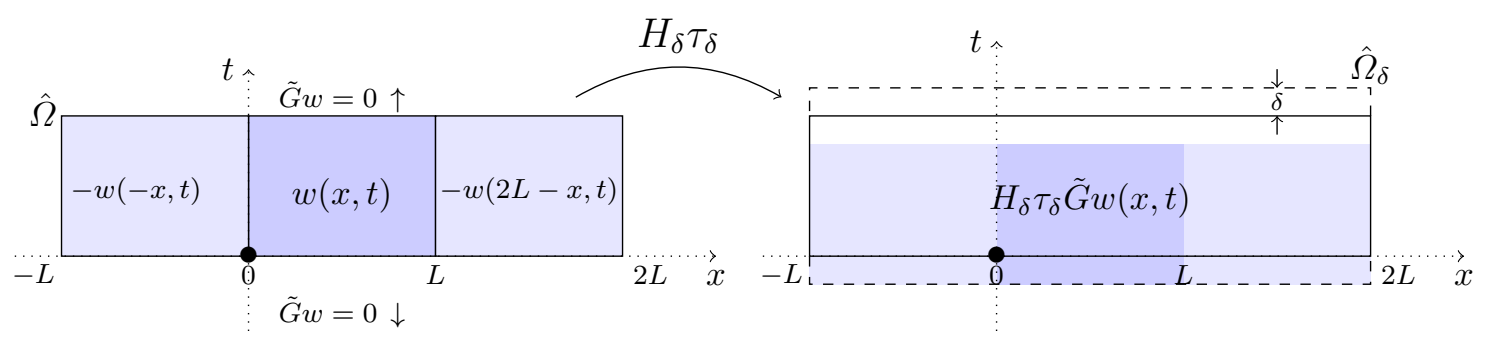

Figure 4.3. Extension and translation in the proof of Theorem 4.2.7.

Hence,

$$
\langle A G v, \varphi\rangle_{\mathcal{D}(\hat{\Omega})}=\left(v, A G^{\prime} \varphi\right)_{\Omega}=\left(A v, G^{\prime} \varphi\right)_{\Omega}-\left\langle D v, G^{\prime} \varphi\right\rangle_{W}
$$

Now observe that $\left.G^{\prime} \varphi\right|_{\Gamma}=0$. Hence by Lemma 4.2.1. $G^{\prime} \varphi$ is in $V$. Since $v \in V^{*}$ and $G^{\prime} \varphi \in V$, the last term of 4.2.8 must vanish by 4.2.3). Thus $\langle A G v, \varphi\rangle_{\mathcal{D}(\hat{\Omega})}=$ $\left(A v, G^{\prime} \varphi\right)_{\Omega}=(G A v, \varphi)_{\hat{\Omega}}$, completing the proof of the claim 4.2.6). In view of 4.2.6), we conclude that $G v$ is in $W(\hat{\Omega})$ whenever $v \in V^{*}$.

Step 2. Translate: Let $\tilde{G} v$ denote the extension of $G v$ by zero to $\mathbb{R}^{2}$, i.e., $\tilde{G} v$ equals $G v$ on $\hat{\Omega}$ and equals zero elsewhere. Let $\tau_{\delta}$ be the translation operator in the $-t$ direction by $\delta$, i.e., $\left(\tau_{\delta} w\right)(x, t)=w(x, t+\delta)$. Its well known $([\mathbf{7}])$ that

$$
\lim _{\delta \rightarrow 0}\left\|\tau_{\delta} g-g\right\|_{\mathbb{R}^{2}}=0, \quad \forall g \in L^{2}\left(\mathbb{R}^{2}\right)
$$

Let $\hat{\Omega}_{\delta}=(-L, 2 L) \times(-\delta, T+\delta)$ and let $H_{\delta}$ denote the restriction of functions on $\mathbb{R}^{2}$ to $\hat{\Omega}_{\delta}$. By a change of variable,

$$
\left(\tau_{\delta} \tilde{G} f, g\right)_{\hat{\Omega}_{\delta}}=\left(G f, \tau_{-\delta} g\right)_{\hat{\Omega}}, \quad \forall f \in L^{2}(\Omega), g \in L^{2}\left(\hat{\Omega}_{\delta}\right)
$$

We now claim that

$$
A H_{\delta} \tau_{\delta} \tilde{G} v=H_{\delta} \tau_{\delta} \tilde{G} A v, \quad \forall v \in V^{*}
$$


Indeed, for any $\varphi \in \mathcal{D}\left(\hat{\Omega}_{\delta}\right)$, the action of the distribution $A H_{\delta} \tau_{\delta} \tilde{G} v$ on $\bar{\varphi}$ equals

$$
\begin{aligned}
\left\langle A H_{\delta} \tau_{\delta} \tilde{G} v, \varphi\right\rangle_{\mathcal{D}\left(\hat{\Omega}_{\delta}\right)} & =\left(\tau_{\delta} \tilde{G} v, A \varphi\right)_{\hat{\Omega}_{\delta}}=\left(G v, A \tau_{-\delta} \varphi\right)_{\hat{\Omega}}=\left(v, G^{\prime} A \tau_{-\delta} \varphi\right)_{\Omega} \\
=\left(v, A G^{\prime} \tau_{-\delta} \varphi\right)_{\Omega} & \\
& =\left(A v, G^{\prime} \tau_{-\delta} \varphi\right)_{\Omega}-\left\langle D v, G^{\prime} \tau_{-\delta} \varphi\right\rangle_{W}
\end{aligned}
$$

where we have used (4.2.10), 4.2.5), and 4.2.7) consecutively. Since $\left.\left(G^{\prime} \tau_{-\delta} \varphi\right)\right|_{\Gamma}=0$, Lemma 4.2.1 shows that $G^{\prime} \tau_{-\delta} \varphi$ is in $V$, and consequently the last term above vanishes for all $v \in V^{*}$. Continuing and using 4.2.5 and 4.2.10 once more, $\left\langle A H_{\delta} \tau_{\delta} \tilde{G} v, \varphi\right\rangle_{\mathcal{D}\left(\hat{\Omega}_{\delta}\right)}=\left(G A v, \tau_{-\delta} \varphi\right)_{\hat{\Omega}}=\left(\tau_{\delta} \tilde{G} A v, \varphi\right)_{\hat{\Omega}_{\delta}}$. This proves 4.2.11.

Step 3. Mollify: Consider the mollifier $\rho_{\varepsilon} \in \mathcal{D}\left(\mathbb{R}^{2}\right)$, for each $\varepsilon>0$, defined by $\rho_{\varepsilon}(x, t)=\varepsilon^{-2} \rho_{1}\left(\varepsilon^{-1} x, \varepsilon^{-1} t\right)$, where

$$
\rho_{1}(x, t)= \begin{cases}k e^{-\frac{1}{1-x^{2}-t^{2}}} & \text { if } x^{2}+t^{2}<1 \\ 0 & \text { if } x^{2}+t^{2} \geq 1\end{cases}
$$

and $k$ is a constant chosen so that $\int_{\mathbb{R}^{2}} \rho_{1}=1$. It is well known $([7])$ that when any function $w$ in $L^{2}\left(\mathbb{R}^{2}\right)$ is convolved with $\rho_{\varepsilon}$, the result $\rho_{\varepsilon} * w$ is infinitely smooth and satisfies

$$
\lim _{\varepsilon \rightarrow 0}\left\|w-\rho_{\varepsilon} * w\right\|_{\mathbb{R}^{2}}=0, \quad \forall w \in L^{2}\left(\mathbb{R}^{2}\right)
$$

Consider any small enough $\delta>0$, say $\delta<\min (L / 2, T / 2)$, and define two functions $v_{\varepsilon}=\rho_{\varepsilon} * \tau_{\delta} \tilde{G} v$ and $a_{\varepsilon}=\rho_{\varepsilon} * \tau_{\delta} \tilde{G} A v$. Note that the two smooth functions $A v_{\varepsilon}$ and $a_{\varepsilon}$ need not coincide everywhere. However, because of 4.2.11), they coincide on $\Omega$ whenever $\varepsilon<\delta / 2$ :

$$
A v_{\varepsilon}=a_{\varepsilon} \quad \text { on } \Omega \text {. }
$$


Let us therefore set $\delta$ to, say, $\delta=3 \varepsilon$ and let $\varepsilon<\min (L / 2, T / 2) / 3$ go to zero. Note that

$$
\begin{aligned}
\left\|A v_{\varepsilon}-A v\right\|_{\Omega} & =\left\|a_{\varepsilon}-A v\right\|_{\Omega}=\left\|\rho_{\varepsilon} * \tau_{\delta} \tilde{G} A v-A v\right\|_{\Omega} \\
& \leq\left\|\rho_{\varepsilon} * \tau_{\delta} \tilde{G} A v-\tau_{\delta} \tilde{G} A v\right\|_{\mathbb{R}^{2}}+\left\|\tau_{\delta} \tilde{G} A v-\tilde{G} A v\right\|_{\mathbb{R}^{2}}, \\
\left\|v_{\varepsilon}-v\right\|_{\Omega} & \leq\left\|\rho_{\varepsilon} * \tau_{\delta} \tilde{G} v-\tau_{\delta} \tilde{G} v\right\|_{\Omega}+\left\|\tau_{\delta} \tilde{G} v-v\right\|_{\Omega} \\
& \leq\left\|\rho_{\varepsilon} * \tau_{\delta} \tilde{G} v-\tau_{\delta} \tilde{G} v\right\|_{\mathbb{R}^{2}}+\left\|\tau_{\delta} \tilde{G} v-\tilde{G} v\right\|_{\mathbb{R}^{2}} .
\end{aligned}
$$

Using (4.2.12) and (4.2.9) it now immediately follows that

$$
\lim _{\varepsilon \rightarrow 0}\left\|v_{\varepsilon}-v\right\|_{W}=0
$$

To conclude, examine the value of $v_{\varepsilon}$ at points $z=(0, t)$ for any $0<t<T$, namely

$$
v_{\varepsilon}(0, t)=\int_{\mathbb{R}} \int_{\mathbb{R}} \rho_{\varepsilon}\left(-x^{\prime}, t-t^{\prime}\right)\left(\tau_{\delta} \tilde{G} v\right)\left(x^{\prime}, t^{\prime}\right) d x^{\prime} d t^{\prime} .
$$

The integrand of the inner integral is the product of an even function $\left(\rho_{\varepsilon}\right)$ of $x^{\prime}$ and an odd function $\tau_{\delta} \tilde{G} v$ of $x^{\prime}$. Hence $v_{\varepsilon}(0, t)=0$. The same holds for points $z=(L, t)$. Moreover, since $\tau_{\delta} \tilde{G} v(y)$ is identically zero in a neighborhood of $z=(T, x)$ for all $0<x<L$, we conclude that $\left.v_{\varepsilon}\right|_{\Gamma^{*}}=0$.

\subsection{Error Estimates for the ideal DPG method}

Continuing to consider the set $\Omega$ as defined in Section 4.2.3, we now proceed to analyze the convergence of the ideal DPG method for Problem 4.2.5. The ideal DPG method finds $u_{h}$ and $q_{h}$ in finite dimensional subspaces $U_{h} \subset L^{2}(\Omega)$ and $Q_{h} \subset Q$ respectively, satisfying

$$
b\left(\left(u_{h}, q_{h}\right), v\right)=F(v), \quad \text { for all } v \in T\left(U_{h} \times Q_{h}\right) .
$$


Here $T: L^{2}(\Omega) \times Q \rightarrow W_{h}$ is defined by $(T(z, r), v)=b((z, r), v)$ for all $v \in W_{h}$ and any $(z, r) \in L^{2}(\Omega) \times Q$ as in Section 1.5. The wellposedness of Problem 4.2.5 follows from Theorem 4.2.6, now that we have verified Assumption11 in Theorem 4.2.7. Hence to obtain convergence rates for specific subspaces, we need only develop interpolation error estimates. Since the interpolation properties of the $L^{2}$-conforming $U_{h}$ are standard, we need only discuss those of $Q_{h}$. To study this, we will create a spacetime finite element space $V_{h} \subset V$, then identify $Q_{h}$ as $D_{h}\left(V_{h}\right)$, and finally establish interpolation estimates for $Q_{h}$ using those for $V_{h}$. Note that $V_{h}$ will be used only in the proof (and not in the computations).

To transparently present the ideas, we shall limit ourselves to the very simple case of a uniform mesh $\Omega_{h}$ of spacetime square elements of side length $h$. Let $\mathcal{E}_{h}$ denote the set of edges of $\Omega_{h}$. On any $E \in \mathcal{E}_{h}$, let $P_{p}(E)$ denote the space of polynomials on the edge of degree at most $p$. On any $K \in \Omega_{h}$, let $Q_{p}(K)$ denote the space of polynomials of degree at most $p$ in $x$ and at most $p$ in $t$. To begin the finite element construction, we consider the reference element $\hat{K}=(0,1) \times(0,1)$ and the element space $Q_{p}(\hat{K})$, endowed with the following degrees of freedom: For any $w \in H^{3}(K)$, and for each $i \in\{0,1, \ldots, p-2\}$ and $j \in\{0,1,2, \ldots, p\}$, write $x_{i}=i /(p-2)$ and $t_{j}=j / p$ and set

$$
\sigma_{i j}(w)=w\left(x_{i}, t_{j}\right), \quad \sigma_{j}^{0}(w)=\partial_{x} w\left(0, t_{j}\right), \quad \sigma_{j}^{1}(w)=\partial_{x} w\left(1, t_{j}\right) .
$$

Together, these form a set $\Sigma$ with $(p-1)(p+1)+2(p+1)$ linear functionals. The triple $\left(\hat{K}, Q_{k}(\hat{K}), \Sigma\right)$ is a unisolvent finite element, in the sense of [13], as we show next.

Lemma 4.3.1. Suppose $p \geq 3$. Then any polynomial $w \in Q_{p}(\hat{K})$ is uniquely defined by the values of its degrees of freedom $\sigma$ in $\Sigma$. 

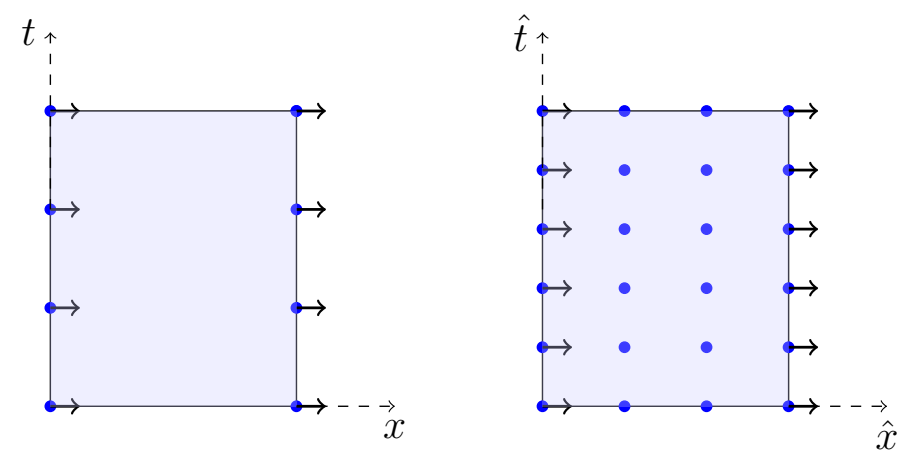

Figure 4.4. Degrees of freedom in the $p=3$ (left) and $p=5$ (right) cases.

Proof. Suppose $w \in Q_{p}(\hat{K})$ and $\sigma(w)=0$ for all $\sigma \in \Sigma$. Then $w_{j}(x)=w\left(x, t_{j}\right)$ is a polynomial of degree $p$ in one variable $(x)$. The Hermite and Lagrange degrees of freedom on $t=t_{j}$ imply $w_{j}=0$. Now, fixing $x$, observe that the polynomial $w(x, t)$ is of degree at most $p$ in the variable $t$ and has $p+1$ zeros. Hence $w \equiv 0$ and the proof is complete since $\operatorname{dim} Q_{p}(\hat{K})$ equals the number of degrees of freedom.

Next, consider the global finite element space $W_{h}^{p}(\Omega)=\left\{w \in L^{2}(\Omega): \partial_{t} w\right.$ and $\partial_{x x} w$ are in $L^{2}(\Omega)$ and $\left.w\right|_{K} \in Q_{p}(K)$ for all $K \in \Omega_{h}$. Each element $K \in \Omega_{h}$ is obtained by mapping the reference element $\hat{K}$ by $T_{K}: \hat{K} \rightarrow K, T_{K}(\hat{x}, \hat{t})=(h \hat{x}+$ $\left.x_{K}, h \hat{t}+t_{K}\right)$, where $\left(x_{K}, t_{K}\right)$ is the lower left corner vertex of $K$, and the element space $Q_{p}(K)$ is the pull back of the reference element space $Q_{p}(\hat{K})$ under this map. The space $W_{h}^{p}(\Omega)$ can be controlled by a global set of degrees of freedom obtained by mapping the reference element degrees of freedom and, as usual, coalescing those that coincide at the mesh element interfaces.

On the reference element $\hat{K}$, the degrees of freedom define an interpolation operator

$$
\hat{\Pi} w=\sum_{\sigma \in \Sigma} \sigma(w) \varphi_{\sigma}
$$


where, as usual, $\left\{\varphi_{\eta} \in Q_{p}(\hat{K}): \eta \in \Sigma\right\}$ is the set of shape functions obtained as the dual basis of $\Sigma$. By the Sobolev inequality in two dimensions, $\hat{\Pi}: H^{3}(\hat{K}) \rightarrow Q_{p}(\hat{K})$ is continuous. Similarly, the global degrees of freedom define an interpolation operator $\Pi: H^{3}(\Omega) \rightarrow W_{h}^{p}(\Omega)$ satisfying

$$
(\Pi w) \circ T_{K}=\hat{\Pi}\left(w \circ T_{K}\right)
$$

Lemma 4.3.2. If $w \in H^{p+1}(\Omega)$, then for all $p \geq 3$,

$$
\begin{aligned}
\|w-\Pi w\|_{\Omega} & \leq C h^{p+1}|w|_{H^{p+1}(\Omega)} \\
\left\|\partial_{t}(w-\Pi w)\right\|_{\Omega} & \leq C h^{p}|w|_{H^{p+1}(\Omega)} \\
\left\|\partial_{x x}(w-\Pi w)\right\|_{\Omega} & \leq C h^{p-1}|w|_{H^{p+1}(\Omega)} .
\end{aligned}
$$

Proof. Changing variables $(x, t)=T_{K}(\hat{x}, \hat{t})$ as $(\hat{x}, \hat{t})$ runs over $\hat{K}$, integrating, and using 4.3.2,

$$
\begin{aligned}
\|w-\Pi w\|_{K} & =h\|\hat{w}-\hat{\Pi} \hat{w}\|_{\hat{K}} \\
\left\|\partial_{t}(w-\Pi w)\right\|_{K} & =\left\|\partial_{\hat{t}}(\hat{w}-\hat{\Pi} \hat{w})\right\|_{\hat{K}} \\
\left\|\partial_{x x}(w-\Pi w)\right\|_{K} & =h^{-1}\left\|\partial_{\hat{x} \hat{x}}(\hat{w}-\hat{\Pi} \hat{w})\right\|_{\hat{K}} .
\end{aligned}
$$

On the reference element, since $H^{p+1}(\hat{K}) \hookrightarrow H^{3}(\hat{K})$, the interpolation operator $\hat{\Pi}$ : $H^{p+1}(\hat{K}) \rightarrow Q_{p}(\hat{K})$ is continuous. Moreover $\hat{\Pi} \hat{w}=\hat{w}$ for all $\hat{w} \in Q_{p}(\hat{K})$. Hence, the Bramble-Hilbert Lemma yields a $\hat{C}>0$ such that $\|\hat{w}-\hat{\Pi} \hat{w}\|_{H^{3}(\hat{K})} \leq \hat{C}|\hat{w}|_{H^{p+1}(\hat{K})}$ for all $\hat{w} \in H^{p+1}(\hat{K})$. Since $|\hat{w}|_{H^{p+1}(\hat{K})} \leq C h^{p}|w|_{H^{p+1}(K)}$, combining with 4.3.3) and summing over all the elements in $\Omega_{h}$, we obtain the result. 
Now we are ready to present the main result of this section. Set $V_{h}=W_{h}^{p}(\Omega) \cap V$ and

$$
Q_{h}=D_{h}\left(V_{h}\right), \quad U_{h}=\left\{u \in L^{2}(\Omega):\left.u\right|_{K} \in Q_{p-1}(K) \text { for all } K \in \Omega_{h}\right\}
$$

Theorem 4.3.3. Let $p \geq 3$. Suppose $u \in V \cap H^{p+1}(\Omega)$ and $q=D_{h} u$ solve Problem 4.2.5 and suppose $U_{h} \times Q_{h}$ is set by 4.3.4). Then, there exists a constant $C$ independent of $h$ such that the discrete solution $u_{h} \in U_{h}$ and $q_{h} \in Q_{h}$ solving (4.3.1) satisfies

$$
\left\|u-u_{h}\right\|_{\Omega}+\left\|q-q_{h}\right\|_{Q} \leq C h^{r}|u|_{H^{r+2}(\Omega)}
$$

for $2 \leq r \leq p-1$

Proof. By [19, Theorem 2.2] the ideal DPG method is quasioptimal:

$$
\begin{aligned}
\left\|(u, q)-\left(u_{h}, q_{h}\right)\right\|_{U \times Q}^{2} & \leq C \inf _{\left(z_{h}, r_{h}\right) \in U_{h} \times Q_{h}}\left\|(u, q)-\left(z_{h}, r_{h}\right)\right\|_{U \times Q}^{2} \\
& =C \inf _{\left(z_{h}, r_{h}\right) \in U_{h} \times Q_{h}}\left(\left\|u-z_{h}\right\|_{\Omega}^{2}+\left\|q-r_{h}\right\|_{Q}^{2}\right) .
\end{aligned}
$$

Because of the standard approximation estimate $\inf _{z_{h} \in U_{h}}\left\|u-z_{h}\right\|_{\Omega} \leq C h^{r}|u|_{H^{r}(\Omega)}$ for $0 \leq r \leq p-1$, it suffices to focus on $\left\|q-r_{h}\right\|_{Q}$. Since $q=D_{h} u$, by the definition of $Q$-norm 1.4.14, and the fact that any $r_{h}$ in $Q_{h}$ equals $D_{h} v_{h}$ for some $v_{h} \in V_{h}$, we have

$$
\inf _{r_{h} \in Q_{h}}\left\|q-r_{h}\right\|_{Q} \leq \inf _{v_{h} \in V_{h}}\left\|u-v_{h}\right\|_{W} \leq\|u-\Pi u\|_{W}
$$

Applying Lemma 4.3.2, the result follows.

We conclude this chapter by examining a property of $Q_{h}$ that is useful for computations. Let $\mathcal{E}_{h}^{\mid}$and $\mathcal{E}_{h}^{-}$denote the set of vertical and horizontal (closed) mesh edges, 
respectively, and $\mathcal{E}_{h}^{+}=\mathcal{E}_{h}^{-} \cup \mathcal{E}_{h}^{-}$. Let $E_{h}^{\prime}$ and $E_{h}^{+}$denote the closed set formed by the union of all edges in $\mathcal{E}_{h}^{\prime}$ and $\mathcal{E}_{h}^{+}$, respectively. Let $Q_{h}^{\prime}=\left\{r \in L^{2}\left(E_{h}^{\prime}\right):\left.r\right|_{F} \in P_{p}(F)\right.$ for all $\left.F \in \mathcal{E}_{h}^{\prime}\right\}$ and $Q_{h}^{+}=\left\{r \in L^{2}\left(E_{h}^{+}\right): r\right.$ is continuous on $E_{h}^{+}$and $\left.r\right|_{F} \in P_{p}(F)$ for all $F \in \mathcal{E}_{h}^{+}$and $\left.\left.r\right|_{\Gamma}=0\right\}$. For any $v_{h} \in V_{h}$, since $v_{h}$ is a polynomial on each element, we may integrate by parts element by element to get

$$
\begin{aligned}
\left\langle D_{h} v_{h}, \psi\right\rangle_{h} & =\left(A_{h} v_{h}, \psi\right)_{h}-\left(v_{h}, A_{h} \psi\right)_{h} \\
& =\sum_{K \in \Omega_{h}} \int_{\partial K} \hat{\imath} n_{t} v_{h} \bar{\psi}+\int_{\partial K} v_{h} n_{x}\left(\partial_{x} \bar{\psi}\right)-\int_{\partial K} n_{x}\left(\partial_{x} v_{h}\right) \bar{\psi}
\end{aligned}
$$

for all $\psi \in \mathcal{D}(\bar{\Omega})$. Thus $q=D_{h} v_{h}$ satisfies

$$
\langle q, \psi\rangle_{h}=\sum_{K \in \Omega_{h}} \int_{\partial K} q^{+}\left(\hat{\imath} n_{t} \bar{\psi}\right)+\int_{\partial K} q^{+} n_{x}\left(\partial_{x} \bar{\psi}\right)-\int_{\partial K} q^{\prime}\left(n_{x} \bar{\psi}\right)
$$

where $q^{+}=\left.v_{h}\right|_{E_{h}^{+}}$and $q^{\prime}=\left.\partial_{x} v_{h}\right|_{E_{h}^{\prime}}$. In computations, one may therefore identify $Q_{h}$ with the interfacial polynomial space $Q_{h}^{+} \times Q_{h}^{\prime}$ whose components are of degree at most $p$. 


\section{A spacetime DPG method for the first order wave equation}

In this chapter we apply the abstract ultraweak formulation of Chapter 1 to the transient wave problem. The goal is to study the feasibility of the DPG method for the first order spacetime acoustic wave system.

\subsection{Model problem: The transient wave problem}

Let $\Omega_{0}$ be an open subset of $\mathbb{R}^{d-1}$,with $d>1$, representing the spatial domain, with boundary $\partial \Omega_{0}$, and let $\Omega=\Omega_{0} \times(0, T)$ be the spacetime domain, where $T>0$ represents the final time. We consider the first order system for the wave equation given by

$$
\begin{array}{r}
\partial_{t} q-c \nabla_{x} \mu=g, \\
\partial_{t} \mu-c \operatorname{div}_{x} q=f
\end{array}
$$

where $f \in L^{2}(\Omega)$ and $g \in L^{2}(\Omega)^{d-1}$ and $c>0$ denotes the constant wave speed of the material. Here the differential operators $\operatorname{div}_{x}$ and $\nabla_{x}$ represent the divergence and gradient (distributional) operators that differentiate only along the space variable $x$ components. We add homogeneous initial and boundary conditions:

$$
\left.\mu\right|_{t=0}=0,\left.\quad q\right|_{t=0}=0,\left.\quad \mu\right|_{\partial \Omega \times(0, T)}=0 .
$$

Here, $q$ represents the velocity and $\mu$ the pressure. We now cast this problem in the framework of the abstract setting in Chapter 1 . 
5.1.1. The formal wave operator. Formally, the wave operator generated by the above system may be considered as a first order distributional derivative operator. Namely, set $A: L^{2}(\Omega)^{d} \rightarrow \mathcal{D}^{\prime}(\Omega)^{d}$ by

$$
A u=\left[\begin{array}{c}
\partial_{t} u_{q}-c \nabla_{x} u_{\mu} \\
\partial_{t} u_{\mu}-c \operatorname{div}_{x} u_{q}
\end{array}\right]
$$

where $u$ in $L^{2}(\Omega)^{d}$ is block partitioned into

$$
u=\left[\begin{array}{l}
u_{q} \\
u_{\mu}
\end{array}\right], \quad u_{q} \in L^{2}(\Omega)^{d-1}, \quad u_{\mu} \in L^{2}(\Omega) .
$$

Note that this operator satisfies Assumptions A-a and A-b in Chapter 1 when setting $m=l=d$ and $k=1$. Following the framework of Chapter 1 we define

$$
W(\Omega)=\left\{u \in L^{2}(\Omega)^{d}: A u \in L^{2}(\Omega)^{d}\right\}
$$

As in the previous chapters, we abbreviate $W(\Omega)=W$. The formal adjoint of $A$ is the distributional differentiation operator $-A$, whose graph space is equal to $W$ and satisfies

$$
(A w, \tilde{w})=-(w, A \tilde{w}) \quad \text { for all } w, \tilde{w} \in \mathcal{D}(\Omega)^{d}
$$

We define the operator $D: W \rightarrow W^{\prime}$ by

$$
\langle D u, v\rangle_{W}=(A u, v)_{\Omega}+(u, A v)_{\Omega} \quad \text { for all } u, v \in W
$$

where $W^{\prime}$ is the dual space of $W$, and $\langle\cdot, \cdot\rangle_{W}$ represents the duality pairing of a functional in $W^{\prime}$ with a element of $W$. For smooth functions $u, v \in \mathcal{D}(\bar{\Omega})^{d}$, integration 
by parts shows that

$$
\langle D u, v\rangle_{W}=\int_{\partial \Omega} u_{q} \cdot\left(n_{t} v_{q}-c n_{x} v_{\mu}\right)+u_{\mu}\left(n_{t} v_{\mu}-c n_{x} \cdot v_{q}\right) .
$$

Here and throughout, $n=\left(n_{x}, n_{t}\right)$ represents the unit outward normal component to $\Omega$ in $\mathbb{R}^{d}$ and functions in $L^{2}(\Omega)^{d}$, like the $u$ and $v$ above, are block partitioned as in 5.1 .3$)$.

5.1.2. The unbounded wave operator. In order to consider the boundary and initial conditions, we now proceed as suggested in Chapter 1, to define an unbounded operator with a domain that takes these conditions into account. Below, by abusing the notation, we shall denote this unbounded operator also by $A$.

First, let us partition the spacetime boundary $\partial \Omega$ into

$$
\Gamma_{0}=\Omega_{0} \times\{0\}, \quad \Gamma_{T}=\Omega_{0} \times\{T\}, \quad \Gamma_{b}=\partial \Omega_{0} \times[0, T] .
$$

We define the following sets of smooth functions:

$$
\begin{gathered}
\mathcal{V}=\left\{u \in \mathcal{D}(\bar{\Omega})^{d}:\left.u\right|_{\Gamma_{0}}=0,\left.u_{\mu}\right|_{\Gamma_{b}}=0\right\}, \\
\mathcal{V}^{*}=\left\{v \in \mathcal{D}(\bar{\Omega})^{d}:\left.v\right|_{\Gamma_{T}}=0,\left.v_{\mu}\right|_{\Gamma_{b}}=0\right\} .
\end{gathered}
$$

Next, let $A: \operatorname{dom}(A) \subset L^{2}(\Omega)^{d} \rightarrow L^{2}(\Omega)^{d}$ be the unbounded operator in $L^{2}(\Omega)^{d}$ defined by the right hand side of $(5.1 .2)$ with

$$
\operatorname{dom}(A)=\left\{u \in W:\langle D u, v\rangle_{W}=0 \text { for all } v \in \mathcal{V}^{*}\right\}
$$

From (5.1.5), we see that the set of smooth functions $\mathcal{D}(\Omega)^{d}$ is contained in $\operatorname{dom}(A)$, thus Assumption A-c holds. Hence, $A$ is a densely defined operator in $L^{2}(\Omega)^{d}$. Therefore, it has a uniquely defined adjoint $A^{*}$, which is again an unbounded operator. The adjoint $A^{*}$ equals the distributional derivative operator $-A$ when applied to $\operatorname{dom}\left(A^{*}\right)$. 
This domain is prescribed as in standard functional analysis [7] by

$$
\begin{array}{r}
\operatorname{dom}\left(A^{*}\right)=\left\{\tilde{w} \in L^{2}(\Omega)^{d}: \exists \ell \in L^{2}(\Omega)^{d} \text { such that }(A u, \tilde{w})=(u, \ell)\right. \\
\text { for all } u \in \operatorname{dom}(A)\} .
\end{array}
$$

By definition, $\operatorname{dom}(A)$ is a subset of $W(\Omega)$. When this subset is given the topology of $W(\Omega)$, we obtain a closed subset of $W(\Omega)$, which we call $V$, i.e., $V$ and $\operatorname{dom}(A)$ coincide as sets or vector spaces, but not as topological spaces. Note that $\operatorname{dom}\left(A^{*}\right)$ is also a subset of $W$, since for any $\tilde{w} \in \operatorname{dom}\left(A^{*}\right)$, the distribution $-A \tilde{w}$ is in $L^{2}(\Omega)$. Observe that since $V$ is closed, $A$ is a closed operator.

Since A-a, A-b and A-c hold, Lemma 1.3.1 implies

$$
V^{*}={ }^{\perp} D(V) \text {. }
$$

Thus $V^{*}$ is also a closed subset of $W$.

The next observation is that from the definitions of $V$ and the operator $D$ (namely (5.1.8) and (5.1.4) it immediately follows that $\mathcal{V} \subset V$. Note also that if $v^{*} \in \mathcal{V}^{*}$, then $\left(A u, v^{*}\right)=-\left(u, A v^{*}\right)+\langle D u, v\rangle_{W}=-\left(u, A v^{*}\right)$ for all $u \in V$, since $\langle D u, v\rangle_{W}=0$ by the definition of $V$. Therefore $v^{*}$ is in $V^{*}$. To summarize these observations, we have introduced $\mathcal{V}, \mathcal{V}^{*}, V$ and $V^{*}$, satisfying

$$
\mathcal{V} \subset V \quad \text { and } \quad \mathcal{V}^{*} \subset V^{*}
$$

These are the abstract ingredients in the framework of Chapter 1 applied to the wave problem. 


\subsection{The broken weak formulation}

Following the settings of Chapter 1 , we partition the spacetime domain $\Omega$ into a mesh $\Omega_{h}$ of finitely many open elements $K$ such that $\bar{\Omega}=\cup_{K \in \Omega_{h}} \bar{K}$ where $h=$ $\max _{K \in \Omega_{h}} \operatorname{diam}(K)$. The DPG method is based on a variational formulation in a "broken" analogue of $W$, which we call $W_{h}$, defined as in 1.4.2.

We let $A_{h}$ be the wave operator applied element by element, i.e.,

$$
\left.\left(A_{h} w\right)\right|_{K}=A\left(\left.w\right|_{K}\right), \quad w \in W(K), \quad K \in \Omega_{h} .
$$

Let $W_{h}=\left\{w \in L^{2}(\Omega)^{d}: A_{h} w \in L^{2}(\Omega)^{d}\right\}$. The operator $D_{h}: W_{h} \rightarrow W_{h}^{\prime}$ is defined as in 1.4 .3 by

$$
\left\langle D_{h} w, v\right\rangle_{W_{h}}=\left(A_{h} w, v\right)_{\Omega}+\left(w, A_{h} v\right)_{\Omega}
$$

for all $w, v \in W_{h}$, where $\langle\cdot, \cdot\rangle_{W_{h}}$ denotes the duality pairing in $W_{h}$ in accordance with our previous notation. Below we abbreviate $\langle\cdot, \cdot\rangle_{W_{h}}$ to $\langle\cdot, \cdot\rangle_{h}$. Let $D_{h, V}: V \rightarrow W_{h}^{\prime}$ denote the restriction of $D_{h}$ to $V$, i.e., $D_{h, V}=\left.D_{h}\right|_{V}$. The range of $D_{h, V}$, denoted by $Q$, is made into a complete space by the quotient norm

$$
\|\rho\|_{Q}=\inf _{v \in D_{h, V}^{-1}(\{\rho\})}\|v\|_{W}, \quad \rho \in Q \equiv \operatorname{ran}\left(D_{h, V}\right)
$$

We define the bilinear form on $\left(L^{2}(\Omega)^{d} \times Q\right) \times W_{h}$ by

$$
b((v, \rho), w)=-\left(v, A_{h} w\right)_{\Omega}+\langle\rho, w\rangle_{h}
$$

Therefoe "broken" variational formulation for the wave problem now reads as in Problem 1.5.1. Given any $F$ in the dual space $W_{h}^{\prime}$, find $u \in L^{2}(\Omega)^{d}$ and $\lambda \in Q$ such that

$$
b((u, \lambda), w)=F(w) \quad \text { for all } w \in W_{h} .
$$


Critical to the success of any numerical approximation of this formulation, in particular, the DPG approximation, is its wellposedness. By Theorem 1.4.5, this formulation is well-posed, provided we verify

$$
\begin{gathered}
V={ }^{\perp} D\left(V^{*}\right), \\
A: V \rightarrow L^{2}(\Omega)^{d} \text { is a bijection. }
\end{gathered}
$$

Therefore our next focus is on proving (5.2.3) and (5.2.4). Recall from 5.1.10 that $\mathcal{V}$ and $\mathcal{V}^{*}$ are subspaces of smooth functions within $V$ and $V^{*}$. We now show that (5.2.3) and (5.2.4) follow if these are dense subspaces.

\section{TheOrem 5.2.1. Suppose}

$$
\mathcal{V} \text { is dense in } V \text { and } \mathcal{V}^{*} \text { is dense } V^{*}
$$

Then (5.2.3) and (5.2.4) holds. Consequently, the broken weak formulation (5.2.2) is well posed.

Proof. First observe that another way to write 5.1 .8$)$ is

$$
V={ }^{\perp} D\left(\mathcal{V}^{*}\right)
$$

In view of the continuity of $D,(5.1 .10)$, and the assumption that $\mathcal{V}^{*}$ is dense in $V^{*}$, the condition 5.2.3 now immediately follows.

Next, we will prove that

$$
\begin{gathered}
\|u\| \leq 2 T\|A u\|, \quad \text { for all } u \in \mathcal{V}, \\
\|v\| \leq 2 T\left\|A^{*} v\right\|, \quad \text { for all } v \in \mathcal{V}^{*} .
\end{gathered}
$$


These inequalities follow by well-known energy arguments, as shown in [25, Lemma 3]. We briefly include the proof for completeness. Let $v \in \mathcal{V}^{*}$. Then

$$
\begin{aligned}
\|v\|^{2} & =\int_{0}^{T}\left(\int_{\Omega_{0}}|v(x, t)|^{2} d x\right) d t=2 \int_{0}^{T} \int_{T}^{t} \int_{\Omega_{0}} \partial_{s} v(x, s) \cdot v(x, s) d x d s d t \\
& =2 \int_{0}^{T} \int_{t}^{T} \int_{\Omega_{0}} v(x, s) \cdot A^{*} v(x, s) d x d s d t-2 c \int_{0}^{T} \int_{t}^{T} \int_{\partial \Omega_{0}}\left(v_{q} \cdot n_{x}\right) v_{\mu} d x d s d t \\
& \leq 2 T\|v\|\left\|A^{*} v\right\| .
\end{aligned}
$$

The inequality for $\mathcal{V}$ is similarly proved by using its boundary conditions instead of those of $\mathcal{V}^{*}$.

Using the density assumptions, we conclude that (5.2.6) implies

$$
\begin{array}{ll}
\|u\| \leq 2 T\|A u\| & \text { for all } u \in V \text { and } \\
\|v\| \leq 2 T\left\|A^{*} v\right\| & \text { for all } v \in V^{*} .
\end{array}
$$

The conditions of Theorem 1.4 .14 then holds, and this $A$ is a bijection.

Note that the wellposedness result of Theorem 5.2.1, in particular, implies that

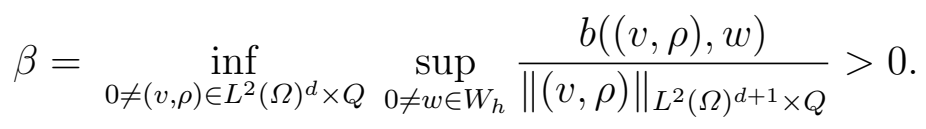

\subsection{Verification of the density condition}

In this section, we verify 5.2 .5 for a simple domain, namely a hyperrectangle (or an orthotope). Accordingly, throughout this section, we fix $\Omega=\Omega_{0} \times(0, T)$ and

$$
\Omega_{0}=\prod_{i=1}^{d}\left(0, a_{i}\right)
$$

for some $a_{i}>0$. While density of smooth functions in general graph spaces can be proved by standard Sobolev space techniques [2], to obtain the density of smooth 
functions with boundary conditions (like those in $\mathcal{V}$ ) we need more arguments. The following proof has some similarities with the proof of Section 4.2.3, an analogous density result for the one-dimensional Schrödinger operator. The main differences from Section 4.2 .3 in the proof below include the consideration of multiple spatial dimensions and the construction of extension operators for vector functions in the wave graph space by combining even and odd reflections appropriately.

Theorem 5.3.1. On the above $\Omega, \mathcal{V}^{*}$ is dense in $V^{*}$ and $\mathcal{V}$ is dense in $V$.

Proof. We shall only prove that $\mathcal{V}$ is dense in $V$, since the proof of the density of $\mathcal{V}^{*}$ in $V^{*}$ is similar. We divide the proof into three main steps.

Step 1. Extension: In this step, we will extend a function in $V$ using spatial reflections to a domain which has larger spatial extent than $\Omega$ (see Figure 5.1).

Let $e_{i}$ denote the standard unit basis vectors in $\mathbb{R}^{d}$. The following operations

$$
R_{i,-} x=x-2 x_{i} e_{i}, \quad R_{i,+} x=x+2\left(a_{i}-x_{i}\right) e_{i}
$$

perform reflections of the spatial coordinate vector $x$ about $x_{i}=0$ and $x_{i}=a_{i}$, for $i=1, \ldots, d$. We set $Q_{0} \equiv \bar{\Omega}$ and then define extended domains $Q_{i}$ in a recursive way, starting from $i=1$ through $i=d$ as follows.

$$
Q_{i,-}=R_{i,-}^{-1} Q_{i-1}, \quad Q_{i,+}=R_{i,+}^{-1} Q_{i-1}, \quad Q_{i}=Q_{i,-} \cup Q_{i-1} \cup Q_{i,+}
$$

The final extended domain is $Q \equiv Q_{d}$. 
Next, we introduce even and odd extensions (in the $x_{i}$-direction) of scalar functions. Namely, let $G_{i, e}, G_{i, o}: L^{2}\left(Q_{i-1}\right) \rightarrow L^{2}\left(Q_{i}\right)$ be defined by

$$
G_{i, e} f(x, t)= \begin{cases}f\left(R_{i,-} x, t\right) & \text { if }(x, t) \in Q_{i,-}, \\ f\left(R_{i,+} x, t\right) & \text { if }(x, t) \in Q_{i,+}, \\ f(x, t) & \text { if }(x, t) \in Q_{i-1},\end{cases}
$$

and

$$
G_{i, o} f(x, t)= \begin{cases}-f\left(R_{i,-} x,, t\right) & \text { if }(x, t) \in Q_{i,-}, \\ -f\left(R_{i,+} x,, t\right) & \text { if }(x, t) \in Q_{i,+}, \\ f(x, t) & \text { if }(x, t) \in Q_{i-1} .\end{cases}
$$

In the case of a vector function $v \in L^{2}\left(Q_{i-1}\right)^{d}$, we define $G_{i} v(x, t)$ to be the extended vector function obtained by extending (in the $i$ th direction) all the components of $v$ using the odd scalar extension, except the $i$ th component, which is extended using the even scalar extension. In other words, for any $i=1, \ldots, d-1$, we define $G_{i}$ : $L^{2}\left(Q_{i-1}\right)^{d} \rightarrow L^{2}\left(Q_{i}\right)^{d}$ by

$$
G_{i} v=\left(G_{i, e} v_{i}\right) e_{i}+\sum_{j \neq i}\left(G_{i, o} v_{j}\right) e_{j}
$$

where the sum runs over all $j=1, \ldots, d$ except $i$. Let $E_{k}=G_{k} \circ G_{k-1} \circ \ldots \circ G_{1}$. The cumulative extension over all spatial directions is thus obtained using $E=E_{d}$. It extends functions in $\bar{\Omega}$ to $Q$.

By change of variable formula for integration, we obtain

$$
\begin{aligned}
& \left(G_{i, o} f, g\right)_{Q_{i}}=\left(f, G_{i, o}^{\prime} g\right)_{Q_{i-1}}, \quad \text { for all } f \in L^{2}\left(Q_{i-1}\right), \quad g \in L^{2}\left(Q_{i}\right) \text {, } \\
& \left(G_{i, e} f, g\right)_{Q_{i}}=\left(f, G_{i, e}^{\prime} g\right)_{Q_{i-1}}, \quad \text { for all } f \in L^{2}\left(Q_{i-1}\right), \quad g \in L^{2}\left(Q_{i}\right) \text {, }
\end{aligned}
$$


where the "folding" operators $G_{i, e / o}^{\prime}: L^{2}\left(Q_{i}\right) \rightarrow L^{2}\left(Q_{i-1}\right)$, that go the reverse direction of the extension operators, are defined by

$$
\begin{aligned}
& G_{i, o}^{\prime} g(x, t)=g(x, t)-g\left(R_{i,-}^{-1} x, t\right)-g\left(R_{i,+}^{-1} x, t\right), \\
& G_{i, e}^{\prime} g(x, t)=g(x, t)+g\left(R_{i,-}^{-1} x, t\right)+g\left(R_{i,+}^{-1} x, t\right) .
\end{aligned}
$$

These scalar folding operators combine to form an analogue for vector functions as in (5.3.3), namely

$$
G_{i}^{\prime} w=\left(G_{i, e}^{\prime} w_{i}\right) e_{i}+\sum_{j \neq i}\left(G_{i, o}^{\prime} w_{j}\right) e_{j}
$$

It satisfies $\left(G_{i} v, w\right)_{Q_{i}}=\left(v, G_{i}^{\prime} w\right)_{Q_{i-1}}$ for all $v \in L^{2}\left(Q_{i-1}\right)^{d}, w \in L^{2}\left(Q_{i}\right)^{d}$, and for each $i$ from 1 to $d-1$. Let $E_{k}^{\prime}=G_{k}^{\prime} \circ G_{k+1}^{\prime} \cdots \circ G_{d}^{\prime}$. Then $E^{\prime}=E_{1}^{\prime}$ folds functions in $Q$ to $\bar{\Omega}$ and is the adjoint of the extension $E$ in the following sense.

$$
(E u, w)_{Q}=\left(u, E^{\prime} w\right)_{\Omega}, \quad \text { for all } u \in L^{2}(\Omega)^{d}, \quad w \in L^{2}(Q)^{d} .
$$

We want to prove that $E v$ is in $W(Q)$ for any $v \in V$. Note that if $w \in L^{2}(\Omega)^{d}$, then $E w$ in $L^{2}(Q)^{d}$, because each $G_{i}$ maps $L^{2}$ functions into $L^{2}$ per (5.3.3). Therefore, in order to prove $E v$ is in $W(Q)$, it only remains to prove that $A(E v)$ is in $L^{2}(Q)^{d}$. Let $\varphi \in \mathcal{D}(Q)^{d}$ (where we abuse the notation and write $\mathcal{D}(Q)$ for $\mathcal{D}\left(Q^{0}\right)$ whenever $Q^{0}$ is the interior of $Q$ ). Using (5.3.6), the action of the distribution $A E v$ on $\varphi$ equals

$$
\langle A E v, \varphi\rangle_{\mathcal{D}(Q)^{d}}=-(E v, A \varphi)_{Q}=-\left(v, E^{\prime} A \varphi\right)_{\Omega}
$$


To analyze the last term, first observe that by the chain rule applied to a smooth scalar function $\phi$ on $Q_{1}$,

$$
\begin{aligned}
& \partial_{t}\left(G_{i, o}^{\prime} \phi\right)=G_{i, o}^{\prime} \partial_{t} \phi, \quad \partial_{i}\left(G_{i, o}^{\prime} \phi\right)=G_{i, e}^{\prime}\left(\partial_{i} \phi\right), \quad \partial_{j}\left(G_{i, o}^{\prime} \phi\right)=G_{i, o}^{\prime}\left(\partial_{j} \phi\right), \\
& \partial_{t}\left(G_{i, e}^{\prime} \phi\right)=G_{i, e}^{\prime} \partial_{t} \phi, \quad \partial_{i}\left(G_{i, e}^{\prime} \phi\right)=G_{i, o}^{\prime}\left(\partial_{i} \phi\right), \quad \partial_{j}\left(G_{i, e}^{\prime} \phi\right)=G_{i, e}^{\prime}\left(\partial_{j} \phi\right),
\end{aligned}
$$

for all $j \neq i$. Combining these appropriately for smooth vector function $\psi$ on $Q_{i}$, we find that

$$
\partial_{t}\left(G_{i}^{\prime} \psi\right)=G_{i}^{\prime} \partial_{t} \psi, \quad c \nabla_{x} G_{i, o}^{\prime} \psi_{\mu}=G_{i}^{\prime}\left(c \nabla_{x} \psi_{\mu}\right), \quad c \operatorname{div}_{x} G_{i}^{\prime} \psi_{q}=G_{i, o}^{\prime}\left(c \operatorname{div} \psi_{q}\right)
$$

Thus, for any $\varphi \in \mathcal{D}(Q)^{d}$ we have $E_{i}^{\prime} A \varphi=A E_{i}^{\prime} \varphi$ for all $i=1, \cdots, d-1$, and in particular

$$
E^{\prime} A \varphi=A E^{\prime} \varphi
$$

Returning to (5.3.7) and using (5.3.8) and (5.1.4),

$$
\langle A E v, \varphi\rangle_{\mathcal{D}(Q)^{d}}=\left(A v, E^{\prime} \varphi\right)_{\Omega}-\left\langle D v, E^{\prime} \varphi\right\rangle_{W(\Omega)}
$$

We shall now show that the last term above vanishes. Since $v$ is in $V$, the last term will vanish by the definition of $V$, provided $E^{\prime} \varphi$ is in $\mathcal{V}^{*}$. To prove that $E^{\prime} \varphi$ is in $\mathcal{V}^{*}$, we only need to verify that $E^{\prime} \varphi$ satisfies the boundary conditions in (5.1.7). Since $\varphi$ is compactly supported in $Q$, we obviously have $\left.\left(E^{\prime} \varphi\right)\right|_{\Gamma_{T}}=0$ as $E^{\prime}$ only involves spatial folding.

Next, we claim that $\left.\left[E^{\prime} \varphi\right]_{\mu}\right|_{\Gamma_{b}}=0$ also. To see this, let $\Gamma^{j}$ denote the two facets of $\partial Q_{j}$ where $x_{j}$ is constant and $\gamma^{j}$ denote the two facets of $\partial Q_{j-1}$ where $x_{j}$ is constant. The value of $G_{d-1, o}^{\prime} \varphi_{\mu}(x, t)$ for any $(x, t)$ in $\gamma^{d-2}$ is the sum of the three terms in 5.3.4, two of which cancel each other, and one of which vanishes because 
$\left.\varphi_{\mu}\right|_{\Gamma^{d-1}}=0$. Thus $\left.\varphi_{\mu}\right|_{\Gamma^{d-1}}=\left.0 \Longrightarrow\left(G_{d, o}^{\prime} \varphi_{\mu}\right)\right|_{\partial Q_{d-2}}=0$ (where we have also used the fact that $\varphi_{\mu}$ vanishes on the remainder $\partial Q_{d-2} \backslash \gamma^{d-2}$ ). The same argument can now be repeated to get that $\left.\left(G_{d-1, o}^{\prime} \varphi_{\mu}\right)\right|_{\Gamma^{d-2}}=\left.0 \Longrightarrow\left(G_{d-2, o}\left(G_{d-1, o} \varphi_{\mu}\right)\right)\right|_{\partial Q_{d-3}}=0$. Continuing similarly, we obtain that $\left[E^{\prime} \varphi\right]_{\mu}=G_{1, o}^{\prime} \circ G_{2, o}^{\prime} \circ \cdots \circ G_{d-1, o}^{\prime} \varphi_{\mu}$ vanishes on $\partial Q_{0}=\Gamma_{b}$. Thus, the last term in (5.3.9) is zero and by (5.3.6) we conclude that

$$
\langle A E v, \varphi\rangle_{\mathcal{D}(Q)^{d}}=(E A v, \varphi)_{Q}
$$

for all $\varphi$ in $\mathcal{D}(Q)^{d}$.

By virtue of (5.3.10), we have proved that for any $v \in V, A E v$ is in $L^{2}(Q)^{d}, A E v$ coincides with $E A v$, and $E v$ is in $W(Q)$.

Step 2. Translation: In this step, we will translate up the previously obtained extension in time coordinate. This will give us room to mollify in the next step. Such a translation step is standard in many density proofs (see e.g., [2]).

Let $v \in V$ and let $\tilde{E} v$ denote the extension of $E v$ by zero to $\mathbb{R}^{d}$, i.e., $\tilde{E} v$ equals $E v$ in $Q$ and it is zero elsewhere. Denote by $\tau_{\delta}$ the translation operator in the $t$-direction by $\delta>0$; i.e. $\left(\tau_{\delta} w\right)(x, t)=w(x, t-\delta)$ for scalar or vector functions $w$. It is well
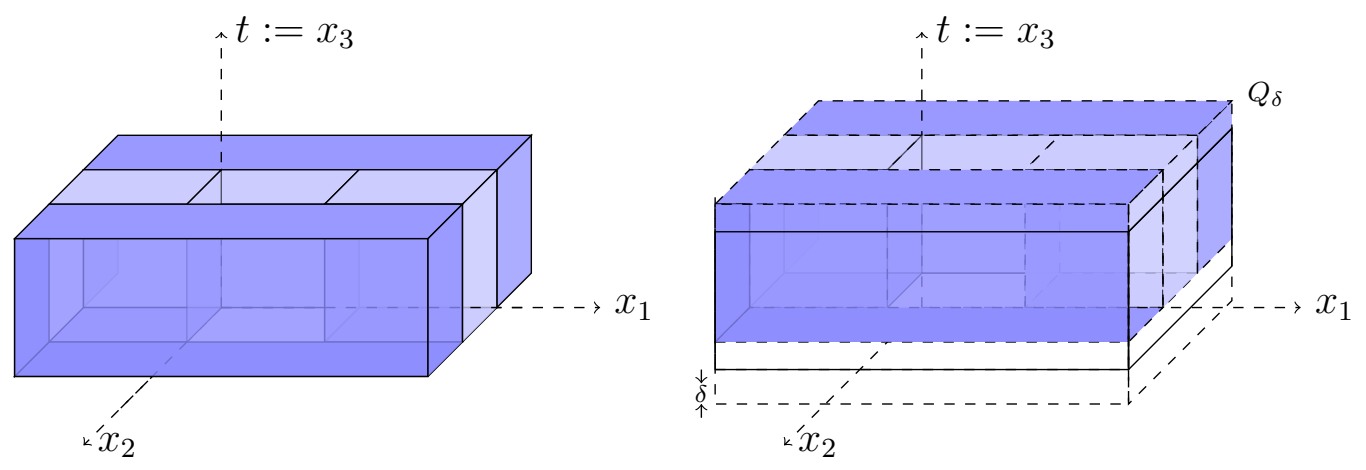

Figure 5.1. Left: Extended domains $Q_{1}$ and $Q_{2}$ when $\Omega \subseteq \mathbb{R}^{3}$. Right: Translation by $\delta$ in the $t$ direction. 
known [7] that

$$
\lim _{\delta \rightarrow 0}\left\|\tau_{\delta} g-g\right\|_{L^{2}\left(\mathbb{R}^{d}\right)}=0, \quad \forall g \in L^{2}\left(\mathbb{R}^{d}\right)
$$

Let $Q_{\delta}=\prod_{i=1}^{i=d-1}\left(-a_{i}, 2 a_{i}\right) \times(-\delta, T+\delta)$ and let $H_{\delta}$ be the restriction from $\mathbb{R}^{d}$ to $Q_{\delta}$.

We will now show that

$$
A H_{\delta} \tau_{\delta} \tilde{E} v=H_{\delta} \tau_{\delta} \tilde{E} A v
$$

By a change of variable,

$$
\left(\tau_{\delta} \tilde{E} w, \tilde{w}\right)_{Q_{\delta}}=\left(E w, \tau_{-\delta} \tilde{w}\right)_{Q}
$$

for all $w \in L^{2}(\Omega)^{d}$ and $\tilde{w} \in L^{2}\left(Q_{\delta}\right)^{d}$. Note that $\left.\tau_{\delta} \tilde{E} v\right|_{\Omega} \in L^{2}(\Omega)$. The distribution $A H_{\delta} \tau_{\delta} \tilde{E} v$ applied to a smooth function $\varphi \in \mathcal{D}\left(Q_{\delta}\right)^{d}$ equals

$$
\left\langle A H_{\delta} \tau_{\delta} \tilde{E} v, \varphi\right\rangle_{\mathcal{D}\left(Q_{\delta}\right)^{d}}=-\left(\tau_{\delta} \tilde{E} v, A \varphi\right)_{Q_{\delta}}=-\left(E v, A \tau_{-\delta} \varphi\right)_{Q}
$$

due to (5.3.13) and the fact that and that $\tau_{-\delta} A \varphi=A \tau_{-\delta} \varphi$. Using also (5.3.6) and 5.3 .8

$$
\begin{aligned}
\left\langle A H_{\delta} \tau_{\delta} \tilde{E} v, \varphi\right\rangle_{\mathcal{D}\left(Q_{\delta}\right)^{d}} & =-\left(v, E^{\prime} A \tau_{-\delta} \varphi\right)_{\Omega}=-\left(v, A E^{\prime} \tau_{-\delta} \varphi\right)_{\Omega} \\
& =\left(A v, E^{\prime} \tau_{-\delta} \varphi\right)_{\Omega}-\left\langle D v, E^{\prime} \tau_{-\delta} \varphi\right\rangle_{W}
\end{aligned}
$$

where we have used (5.3.13), (5.3.6) and (5.3.8) consecutively. Note that $E^{\prime} \tau_{-\delta} \varphi$ satisfies all the boundary conditions required for it to be in $\mathcal{V}^{*}$. Hence the last term in the above display is zero. We therefore conclude that $\left\langle A H_{\delta} \tau_{\delta} \tilde{E} v, \varphi\right\rangle_{\mathcal{D}\left(Q_{\delta}\right)^{d}}=$ $\left(\tau_{\delta} E A v, \varphi\right)_{Q_{\delta}}$, which proves 5.3.12). In particular, $H_{\delta} \tau_{\delta} \tilde{E} v \in W\left(Q_{\delta}\right)$ whenever $v \in$ V. 
Step 3. Mollification: In this step we finish the proof by considering a $v \in V$ and mollifying the time-translated extension $\tau_{\delta} \tilde{E} v$ constructed above.

To recall the standard symmetric mollifier, let $\rho_{\epsilon} \in \mathcal{D}\left(\mathbb{R}^{d}\right)$, for each $\varepsilon>0$ be defined by

$$
\rho_{\varepsilon}(x, t)=\varepsilon^{-d} \rho_{1}\left(\varepsilon^{-1} x, \varepsilon^{-1} t\right)
$$

where

$$
\rho_{1}(x, t)= \begin{cases}k \exp \left(-\frac{1}{1-|x|^{2}-t^{2}}\right) & \text { if }|x|^{2}+t^{2}<1 \\ 0 & \text { if }|x|^{2}+t^{2} \geq 1\end{cases}
$$

and $k$ is a constant chosen so that $\int_{\mathbb{R}^{d}} \rho_{1}=1$. Here $|\cdot|$ is the euclidean norm in $\mathbb{R}^{d}$. Let $\rho_{\varepsilon} * v$ denote the function obtained by component-wise convolution, i.e, $\left[\rho_{\varepsilon} * v\right]_{j}=[v]_{j} * \rho_{\varepsilon}$ for all $j$-components. Then $\rho_{\varepsilon} * v$ is a infinitely smooth vector function that satisfies

$$
\lim _{\varepsilon \rightarrow 0}\left\|v-\rho_{\varepsilon} * v\right\|_{\mathbb{R}^{d}}=0, \quad \forall v \in L^{2}\left(\mathbb{R}^{d}\right)^{d}
$$

Consider any

$$
0<\delta<\min _{1 \leq i \leq d-1}\left(a_{i} / 2, T / 2\right)
$$

and define two functions $v_{\varepsilon}=\rho_{\varepsilon} * \tau_{\delta} \tilde{E} v$ and $a_{\varepsilon}=\rho_{\varepsilon} * \tau_{\delta} \tilde{E} A v$. Note that the $A v_{\varepsilon}=a_{\varepsilon}$ on $\Omega$ whenever $\varepsilon<\delta / 2$, thanks to 5.3 .12 .

We now proceed to show that

$$
\lim _{\varepsilon \rightarrow 0}\left\|\left.v_{\varepsilon}\right|_{\Omega}-v\right\|_{W}=0
$$


Set $\delta=3 \varepsilon$ and let $\varepsilon<\min _{1 \leq i \leq d-1}\left(a_{i} / 2, T / 2\right) / 3$ go to zero. Note that

$$
\begin{aligned}
\left\|A v_{\varepsilon}-A v\right\| & =\left\|a_{\varepsilon}-A v\right\|=\left\|\rho_{\varepsilon} * \tau_{\delta} \tilde{E} A v-A v\right\|_{\Omega} \\
& \leq\left\|\rho_{\varepsilon} * \tau_{\delta} \tilde{E} A v-\tilde{E} A v\right\|_{\mathbb{R}^{d}} \\
& \leq\left\|\rho_{\varepsilon} * \tau_{\delta} \tilde{E} A v-\tau_{\delta} \tilde{E} A v\right\|_{\mathbb{R}^{d}}+\left\|\tau_{\delta} \tilde{E} A v-\tilde{E} A v\right\|_{\mathbb{R}^{d}} \\
\left\|v_{\varepsilon}-v\right\| & \leq\left\|\rho_{\varepsilon} * \tau_{\delta} \tilde{E} v-\tau_{\delta} \tilde{E} v\right\|_{\Omega}+\left\|\tau_{\delta} \tilde{E} v-v\right\|_{\Omega} \\
& \leq\left\|\rho_{\varepsilon} * \tau_{\delta} \tilde{E} v-\tau_{\delta} \tilde{E} v\right\|_{\mathbb{R}^{d}}+\left\|\tau_{\delta} \tilde{E} v-\tilde{E} v\right\|_{\mathbb{R}^{d}} .
\end{aligned}
$$

Using (5.3.11) and 5.3.14) it now immediately follows that 5.3.15) holds.

To conclude, it suffices to prove that $\left.v_{\varepsilon}\right|_{\Omega}$ is in $\mathcal{V}$. Clearly, $\tau_{\delta} \tilde{E} v$ is identically zero in a neighborhood of $\Gamma_{0}$. Hence we conclude that $v_{\varepsilon}=\rho_{\varepsilon} * \tau_{\delta} \tilde{E} v$ vanishes on $\Gamma_{0}$ for small enough $\varepsilon$. Next, let us examine the value of $\left[v_{\varepsilon}\right]_{\mu}$ at points $(x, t)$ on $\Gamma_{b}$, namely

$$
\left[v_{\varepsilon}\right]_{\mu}(x, t)=\int_{\mathbb{R}} \int_{\mathbb{R}^{d-1}} \rho_{\varepsilon}\left(x-x^{\prime}, t-t^{\prime}\right)\left[\tau_{\delta} \tilde{E} v\right]_{\mu}\left(x^{\prime}, t^{\prime}\right) d x^{\prime} d t^{\prime} .
$$

Note that $\rho_{\varepsilon}\left(x-x^{\prime}, t-t^{\prime}\right)$ is a symmetric function of $x^{\prime}$ about $x$. The other term in the

integrand, namely $\left[\tau_{\delta} \tilde{E} v\right]_{\mu}\left(x^{\prime}, t^{\prime}\right)$, is odd about every facet of $\Gamma_{b}$. Hence the integral of their product vanishes whenever $(x, t) \in \Gamma_{b}$. Thus, $\left.\left[v_{\varepsilon}\right]_{\mu}\right|_{\Gamma_{b}}=0$ and $v_{\varepsilon} \in \mathcal{V}$.

\subsection{The method and its error estimates}

In this section, we present the approximation of the previously described broken weak formulation by the (ideal) DPG method and provide a priori and a posteriori error estimates.

5.4.1. The DPG method. We recall the ideal DPG setting of Section 1.5 in terms of $u_{h}, \lambda_{h}$ and $w_{h}$. Namely, we seeks $u_{h}$ and $\lambda_{h}$ in finite-dimensional subspaces 
$U_{h} \subset L^{2}(\Omega)^{d}$ and $Q_{h} \subset Q$, respectively, satisfying

$$
b\left(\left(u_{h}, \lambda_{h}\right), w_{h}\right)=F\left(w_{h}\right), \quad \text { for all } w_{h} \in T\left(U_{h} \times Q_{h}\right) .
$$

where $T: L^{2}(\Omega)^{d} \times Q \rightarrow W_{h}$ is such that $\left.(T(v, \rho), w)_{W}\right)_{W_{h}}=b((v, \rho), w)$ for all $w \in W_{h}$ and any $(v, \rho) \in L^{2}(\Omega)^{d} \times Q$. Hereon we denote $U$ to be $L^{2}(\Omega)^{d}$ and abbreviate the $W_{h}$ inner product $(\cdot, \cdot)_{W_{h}}$ to simply $(\cdot, \cdot)_{h}$.

We rewrite the mixed system introduced in 1.5.1) in terms of $\varepsilon_{h} \in W_{h}, u_{h}, \lambda_{h}$ given by

$$
\left(\varepsilon_{h}, w\right)_{h}=(f, w)-b\left(\left(u_{h}, \lambda_{h}\right), w\right), \quad \text { for all } w \in W_{h} .
$$

One of the two equations in the mixed formulation given below is a restatement of this defining equation for $\varepsilon_{h}$. The mixed formulation seeks $\varepsilon_{h} \in W_{h}$ and $\left(u_{h}, \lambda_{h}\right) \in$ $\left(U_{h} \times Q_{h}\right)$ such that

$$
\begin{aligned}
\left(\varepsilon_{h}, w\right)_{h}+ & b\left(\left(u_{h}, \lambda_{h}\right), w\right)=F(w) & & \text { for all } w \in W_{h}, \\
& b\left((v, \rho), \varepsilon_{h}\right)=0 & & \text { for all }(v, \rho) \in U_{h} \times Q_{h} .
\end{aligned}
$$

We think of

$$
\eta=\left\|\varepsilon_{h}\right\|_{W_{h}} \equiv\left(\sum_{K \in \Omega_{h}}\left\|\varepsilon_{h}\right\|_{W(K)}^{2}\right)^{1 / 2}
$$

as an a posteriori error estimator because $\varepsilon_{h}$ can be computed from (5.4.2) after $u_{h}$ and $\lambda_{h}$ has been computed. Alternately, one can view $\varepsilon_{h}$ as one of the unknowns together with $u_{h}$ and $\lambda_{h}$ as in (5.4.3). Note that (5.4.2) implies

$$
\eta=\sup _{w \in W_{h}} \frac{b\left(\left(u-u_{h}, \lambda-\lambda_{h}\right), w\right)}{\|w\|_{W_{h}}},
$$


so it immediately follows that the estimator is globally reliable and efficient, namely

$$
\beta\left\|\left(u-u_{h}, \lambda-\lambda_{h}\right)\right\|_{U \times Q} \leq \eta \leq\|b\|\left\|\left(u-u_{h}, \lambda-\lambda_{h}\right)\right\|_{U \times Q}
$$

where $\beta$ is as in (5.2.8). In practice, we use element-wise norms of $\varepsilon_{h}$ as a posteriori element error indicator.

To give an a priori error estimate with convergence rates, we need to specify all the approximation subspaces. We choose the space $Q_{h} \subset Q$ by first selecting a finite element space $V_{h} \subset V$ and then applying $D_{h}$ to all functions in it, namely

$$
Q_{h}=D_{h} V_{h}
$$

This way we guarantee that $Q_{h}$ is a subspace of $Q$. The definition of $V_{h}$ and the finite element subspaces of $U$ are based on the type of elements in $\Omega_{h}$. We consider two cases:

Case $\mathbf{A}: \Omega_{h}$ is a geometrically conforming mesh of $(d+1)$-simplices:

$$
\begin{aligned}
& V_{h}=\left\{u \in V \cap C(\bar{\Omega})^{d}:\left.u\right|_{K} \in P_{p+1}(K)^{d} \text { for all } K \in \Omega_{h}\right\} \\
& U_{h}=\left\{u \in L^{2}(\Omega)^{d}:\left.u\right|_{K} \in P_{p}(K)^{d} \text { for all } K \in \Omega_{h}\right\}
\end{aligned}
$$

where $P_{p}(K)$ is the space of polynomials of total degree $\leq p$ on $K$.

Case B: $\Omega_{h}$ is a geometrically conforming mesh of hyperrectangles.

$$
\begin{aligned}
& V_{h}=\left\{u \in V \cap C(\bar{\Omega})^{d}:\left.u\right|_{K} \in Q_{p+1}(K)^{d} \text { for all } K \in \Omega_{h}\right\} \\
& U_{h}=\left\{u \in L^{2}(\Omega)^{d}:\left.u\right|_{K} \in Q_{p}(K)^{d}\right\}
\end{aligned}
$$

where $Q_{p}(K)$ is the space of polynomials on $K$ that are of degree at most $p$ in each variable. 
Since the wave operator $A$ is a first order differential operator, $H^{1}(\Omega)^{d} \subset W(\Omega)$. Hence, the Lagrange finite element space $V_{h}$ is contained in $W$. The space $V_{h}$ has a nodal interpolation operator $I_{h}: H^{s+1}(\Omega)^{d} \rightarrow V_{h}$ which is bounded for $s+1>d / 2$, which we shall use in the proof below. We will use $c$ to denote a generic meshindependent constant whose value at different occurrences may differ. Note that in the estimate of the theorem below, $h$ is the discretization parameter in both space and time.

Theorem 5.4.1. Suppose $u \in V \cap H^{s+1}(\Omega)^{d}$ and $\lambda=D_{h} u$ solve (5.2.2). Suppose also that $U_{h}$ and $V_{h}$ are set as in (5.4.4) or (5.4.5) depending on the mesh type, and $Q_{h}=D_{h} V_{h}$. Then, there exists a constant $C>0$ independent of $h$ such that the discrete solution $u_{h} \in U_{h}$ and $\lambda_{h} \in Q_{h}$ solving (5.4.1) satisfies

$$
\left\|u-u_{h}\right\|+\left\|\lambda-\lambda_{h}\right\|_{Q} \leq C h^{s}|u|_{H^{s+1}(\Omega)^{d}}
$$

for $d / 2<s \leq p+1$.

Proof. The ideal DPG method is quasioptimal, i.e., by [19, Theorem 2.2],

$$
\begin{aligned}
\left\|(u, \lambda)-\left(u_{h}, \lambda_{h}\right)\right\|_{U \times Q}^{2} & \leq C \inf _{\left(v_{h}, \rho_{h}\right) \in U_{h} \times Q_{h}}\left\|(u, \lambda)-\left(v_{h}, \rho_{h}\right)\right\|_{U \times Q}^{2} \\
& \leq C \inf _{\left(v_{h}, \rho_{h}\right) \in U_{h} \times Q_{h}}\left\|u-v_{h}\right\|^{2}+\left\|\lambda-\rho_{h}\right\|_{Q}^{2} .
\end{aligned}
$$

The well-known best approximation estimates for $U_{h}$ imply

$$
\inf _{v_{h} \in U_{h}}\left\|u-v_{h}\right\| \leq C h^{s}|u|_{H^{s}(\Omega)^{d+1}} \text {, for all } 0<s \leq p+1
$$


To estimate the remaining term, choose $\rho_{h}=D_{h} I_{h} u$. Then, since $\lambda=D_{h} u$, by the definition of the $Q$-norm in (5.2.1) and the Bramble-Hilbert lemma,

$$
\begin{aligned}
\inf _{\rho_{h} \in Q_{h}}\left\|\lambda-\rho_{h}\right\|_{Q} & \leq\left\|u-I_{h} u\right\|_{W} \\
& \leq C\left\|u-I_{h} u\right\|_{H^{1}(\Omega)^{d}} \leq C h^{s}|u|_{H^{s+1}(\Omega)^{d+1}}
\end{aligned}
$$

for any $u \in H^{s+1}(\Omega)$, for $(d-1) / 2<s \leq p+1$. Thus, from (5.4.7) and (5.4.8), we have that (5.4.6) holds.

\subsection{Implementation and numerical results}

We finish this chapter with numerical results of the DPG discretization in the form (5.4.3) with the following change. Since $W_{h}$ is infinite-dimensional, in order to get a practical method, we must replace $W_{h}$ by a sufficiently rich finite-dimensional space $Y_{h}^{r}$. A full theoretical analysis of this practical realization of the ideal DPG method is currently open, but we will provide numerical studies showing its efficacy in this section. For some integer $r$, set $Y_{h}^{r}$ as follows.

- In Case A (see (5.4.4)) we set $Y_{h}^{r}=\left\{w \in W_{h}(\Omega):\left.w\right|_{K} \in P_{r}(K)^{d}\right\}$,

- In Case B (see (5.4.5)) we set $Y_{h}^{r}=\left\{w \in W_{h}(\Omega):\left.w\right|_{K} \in Q_{r}(K)^{d}\right\}$.

Then, we compute $e_{h} \in Y_{h}^{r}, u_{h} \in U_{h}$ and $\lambda_{h} \in Q_{h}$ satisfying

$$
\begin{array}{rlrl}
\left(e_{h}, w\right)_{h}+ & b\left(\left(u_{h}, \lambda_{h}\right), w\right)=F(w) & & \text { for all } w \in Y_{h}^{r}, \\
b\left((v, \rho), e_{h}\right)=0 & & \text { for all }(v, \rho) \in U_{h} \times Q_{h} .
\end{array}
$$

In our numerical experience, the choice $r=p+d+1$ gave optimal convergence rates (as reported in detail below). This choice is motivated by the study in [38]. The choice $r=p+d-1$ did not give optimal convergence rates for $p>2$ and $d=2$. A brief report of the performance of an adaptive algorithm is also included in the $d=2$ case. Here again, we observed marked deterioration of adaptivity if $r=p+d-1$ 
is used instead of $r=p+d$ for higher degrees. Beyond these comments, we shall not describe these negative results further, but will henceforth focus solely on the $r=p+d+1$ case. All the numerical results have been implemented using the NGSolve [60] finite element software and the codes used for the experiments below are available in [26].

5.5.1. A null space. In order to implement 5.5.1), one strategy is to set $\lambda_{h}=$ $D_{h} z_{h}$ for some $z_{h} \in V_{h}$ and solve

$$
\begin{array}{rlrl}
\left(e_{h}, w\right)_{h}+ & b\left(\left(u_{h}, D_{h} z_{h}\right), w\right)=F(w) & & \text { for all } w \in Y_{h}^{r}, \\
b\left(\left(v, D_{h} r\right), e_{h}\right)=0 & & \text { for all } v \in U_{h}, r \in V_{h} .
\end{array}
$$

We can decompose $V_{h}$ into interior "bubbles" in $V_{h}^{0}=\left\{z \in V_{h}:\left.z\right|_{\partial K}=0\right.$ for all $\left.K \in \Omega_{h}\right\}$, and a remainder $V_{h}^{1} \equiv V_{h} / V_{h}^{0}$. Since $b\left(\left(v, D_{h} V_{h}^{0}\right), w\right)=0$, we may replace $V_{h}$ by $V_{h}^{1}$ in (5.5.2) (and compute a $z_{h} \in V_{h}^{1}$ ). Let $\left\{y_{k}\right\},\left\{u_{i}\right\}$, and $\left\{z_{j}\right\}$ denote a local finite element basis for $Y_{h}^{r}, U_{h}$ and $V_{h}^{1}$, respectively. Using this basis, the system (5.5.2) with $V_{h}$ replaced by $V_{h}^{1}$, yields a matrix equation of the following form

$$
\left[\begin{array}{cc}
A & B \\
B^{T} & 0
\end{array}\right]\left[\begin{array}{l}
e \\
x
\end{array}\right]=\left[\begin{array}{l}
f \\
0
\end{array}\right],
$$

where $\mathrm{e}$ and $\mathrm{x}$ are the vectors of coefficients in the basis expansion of $e_{h} \in Y_{h}^{r}$ and $\left(u_{h}, z_{h}\right) \in U_{h} \times V_{h}$, respectively, $\mathrm{A}_{k l}=\left(y_{l}, y_{k}\right)_{h},\left[\mathrm{~B}_{0}\right]_{k i}=b\left(\left(u_{i}, 0\right), y_{k}\right),\left[\mathrm{B}_{1}\right]_{k j}=$ $b\left(\left(0, D_{h} z_{j}\right), y_{k}\right)$, and $\mathrm{B}=\left[\mathrm{B}_{0}, \mathrm{~B}_{1}\right]$. In all our numerical experiments, for the abovementioned choice of $r=p+d+1$, we observed that the matrices $\mathrm{A}$ and $\mathrm{B}_{0}$ have trivial null spaces.

However, we caution that $B_{1}$ may have a null space. This runs contrary to our experience with DPG methods on non-spacetime problems, so we expand on it. Note 
that (cf. (5.1.5))

$$
\left[\mathrm{B}_{1}\right]_{k j}=b\left(\left(0, D_{h} z_{j}\right), y_{k}\right)=\sum_{K \in \Omega_{h}} \int_{\partial K} \mathrm{D}_{\mathrm{x}, \mathrm{t}} z_{j} \cdot y_{k}
$$

where

$$
\mathrm{D}_{\mathrm{x}, \mathrm{t}}=\left[\begin{array}{cc}
n_{t} & -c n_{x} \\
-c n_{x}^{T} & n_{t}
\end{array}\right] .
$$

It is immediate that on mesh facets with certain combinations of $n_{x}$ and $n_{t}$, the matrix $\mathrm{D}_{\mathrm{x}, \mathrm{t}}$ is singular. Then $\mathrm{B}_{1}$ will have a nontrivial kernel.

As an example, in Figure 5.2, we show one of the $z_{j}$ that is in the null space of $\mathrm{B}_{1}$ on a triangular mesh for $p=1$ and $c=1$. In fact, on the mesh shown, there are 8 basis functions of $V_{h}^{1}$ that are in the null space of $\mathrm{B}_{1}$, two for each diagonal edge. Recall that the wave speed is 1 for our model wave problem, so these edges align with the light cone for $d=2$. In the case of $d=3$ space dimensions, we continued to find a nontrivial null space for $B_{1}$ on analogous meshes.

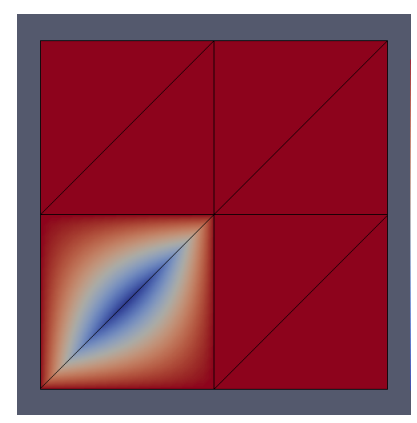

Figure 5.2. Example of a spacetime shape function $z_{j}$ in the kernel

This null space problem occurs because the interface variable $\lambda_{h}$ is set indirectly by applying the singular operator $D_{h}$ on $V_{h}$. If one could directly construct a basis for $Q_{h}=D_{h} V_{h}$, then one can directly implement (5.5.1) (instead of (5.5.2)). However, 
we do not know how to construct such a basis easily on general simplicial meshes. Hence we continue on to describe how to solve (5.5.2) despite its kernel.

5.5.2. Techniques to solve despite the null space. Despite the above- mentioned problem, one may solve the DPG system using one of the following approaches.

5.5.2.1. Technique 1: Remaining orthogonal to null space in conjugate gradients. The matrix system $(5.5 .3)$ can be solved by reducing it to its Schur complement

$$
B^{T} A^{-1} B x=B^{T} A^{-1} f
$$

first. Let $\mathrm{C}=\mathrm{B}^{\mathrm{T}} \mathrm{A}^{-1} \mathrm{~B}$ and $\mathrm{g}=\mathrm{B}^{\mathrm{T}} \mathrm{A}^{-1} \mathrm{f}$. The matrix $\mathrm{C}$ is symmetric and positive semi-definite. Its easy to see that

$$
\operatorname{ker} \mathrm{C}=\operatorname{ker} \mathrm{B} \text {. }
$$

Thus solutions of (5.5.4) are defined only up to this kernel. Note however that since ker $\mathrm{B}=\operatorname{ker} \mathrm{B}_{1}$ and $\mathrm{B}_{0}$ has only the trivial kernel, the $U_{h}$-component of the $\mathrm{DPG}$ solution is uniquely defined independently of ker $\mathrm{B}_{1}$.

One may obtain one solution of 5.5.4 using the conjugate gradient method, which computes its $n$th iterate $\mathbf{x}_{n}$ in the Krylov space

$$
K_{n}\left(\mathrm{C}, \mathrm{r}_{0}\right)=\operatorname{span}\left\{\mathrm{C}^{\mathrm{k}} \mathrm{r}^{0}: k=0, \ldots, n-1\right\}
$$

where $\mathrm{r}_{0}=\mathrm{g}-\mathrm{Ax}_{0}$ is the initial residual. This iteration will converge if $K_{n}\left(\mathrm{C}, \mathrm{r}_{0}\right)$ remains $\left(\ell^{2}\right)$ orthogonal to $\operatorname{ker}(\mathrm{C})$ for all $n$. A simple prescription to guarantee this orthogonality is to choose the initial iterate $\mathrm{x}_{0}=0$. Indeed, if $\mathrm{x}_{0}=0$, then $\mathrm{r}_{0}=\mathrm{g}=$ $\mathrm{B}^{\mathrm{T}} \mathrm{A}^{-1} \mathrm{f}$ is in the range of $\mathrm{B}^{\mathrm{T}}$ which equals the orthogonal complement of ker $\mathrm{B}=\operatorname{ker} \mathrm{C}$. Then for all $n \geq 1$, its obvious that $\mathrm{C}^{n} \mathrm{r}_{0}$ is also orthogonal to ker C. Thus $K_{n}\left(\mathrm{C}, \mathrm{r}_{0}\right)$ is orthogonal to ker C. 
To summarize this technique, we use the conjugate gradient algorithm to compute one solution orthogonal to $\operatorname{ker}(\mathrm{C})$ and extract the unique $U_{h}$-component from that solution for reporting the errors.

5.5.2.2. Technique 2: Regularization of the linear system. Another technique to solve the singular system 5.5.4 approximately is regularization. First, we rewrite 5.5.4 in block form as

$$
\left[\begin{array}{cc}
B_{0}^{T} A^{-1} B_{0} & B_{0}^{T} A^{-1} B_{1} \\
B_{1}^{T} A^{-1} B_{0} & B_{1}^{T} A^{-1} B_{1}
\end{array}\right] x=g .
$$

Since only $\mathrm{B}_{1}$ may have a nontrivial kernel in $V_{h}^{1}$, we can convert this to an invertible system by adding a small positive-definite term in $V_{h}^{1}$. Namely, let $\mathrm{M}$ be the mass matrix $\mathrm{M}_{j l}=\left(z_{l}, z_{j}\right)$. Instead of solving (5.5.4), we solve for

$$
\left[\begin{array}{cc}
B_{0}^{T} A^{-1} B_{0} & B_{0}^{T} A^{-1} B_{1} \\
B_{1}^{T} A^{-1} B_{0} & B_{1}^{T} A^{-1} B_{1}+\alpha M
\end{array}\right] x=g .
$$

where $\alpha$ is a positive regularization parameter, usually set much smaller than the order of the expected discretization errors. In all our reported experiments it was set to $10^{-9}$. The regularized system (5.5.5) is invertible and can be solved using any direct or iterative methods.

5.5.3. Convergence rates in two-dimensional spacetime. Let $\Omega=(0,1)^{2}$. We consider a problem with homogeneous boundary and initial conditions where the exact solution to the second order wave equation is given by $\phi(x, t)=\sin (\pi x) \sin ^{2}(\pi t)$. Then, the exact solution for the first order system is

$$
u=\left[\begin{array}{l}
c \pi \cos (\pi x) \sin ^{2}(\pi t) \\
\pi \sin (\pi x) \sin (2 \pi t)
\end{array}\right]
$$




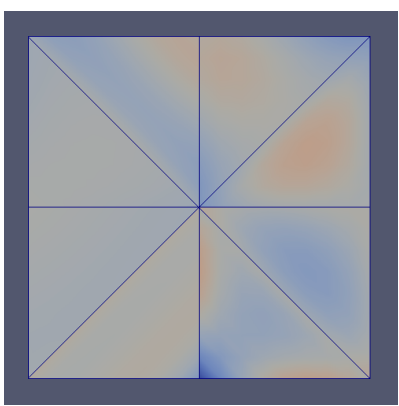

(a) 0 refinements

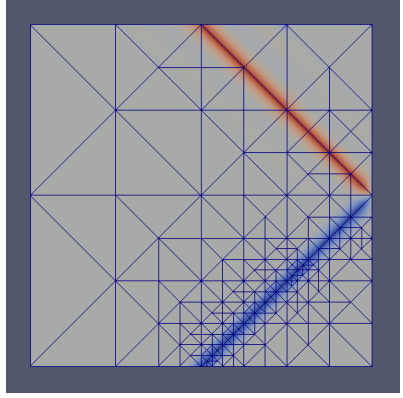

(c) 14 refinements

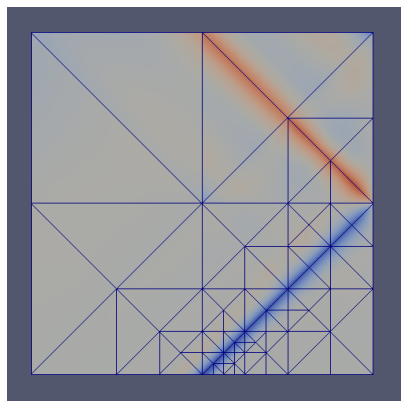

(b) 6 refinements

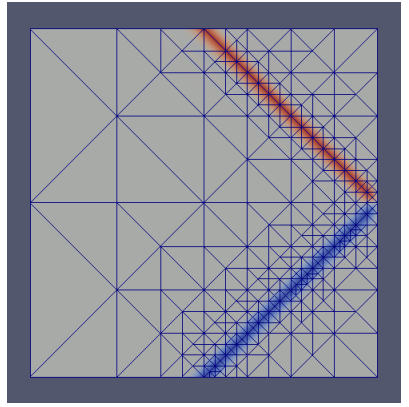

(d) 22 refinements

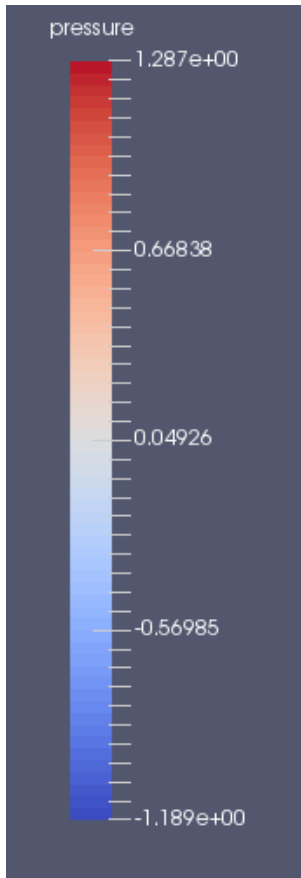

Figure 5.3. Iterates from the adaptive algorithm. Numerical pressure $\mu$ is shown for $p=3$. Time axis is vertical.

and the corresponding source terms are

$$
g=0, \quad f=\pi^{2} \sin (\pi x)\left(2 \cos (2 \pi t)+c^{2} \sin ^{2}(\pi t)\right)
$$

In each experiment, a (non-uniform) coarse triangular mesh of $\Omega$ was constructed, with element diameters not exceeding a reported mesh size $h$, with $c=1$. Successive refinements of the mesh were obtained by connecting the mid points of the edges.

We observe in Table 5.5 that the order of convergence for $u_{h}$ in the $L^{2}$ norm is $O\left(h^{p+1}\right)$ in accordance with Theorem 5.4.1. Similarly in Table 5.5, we observe the same convergence rates for rectangular meshes. All results in both tables were obtained using Technique 1. 

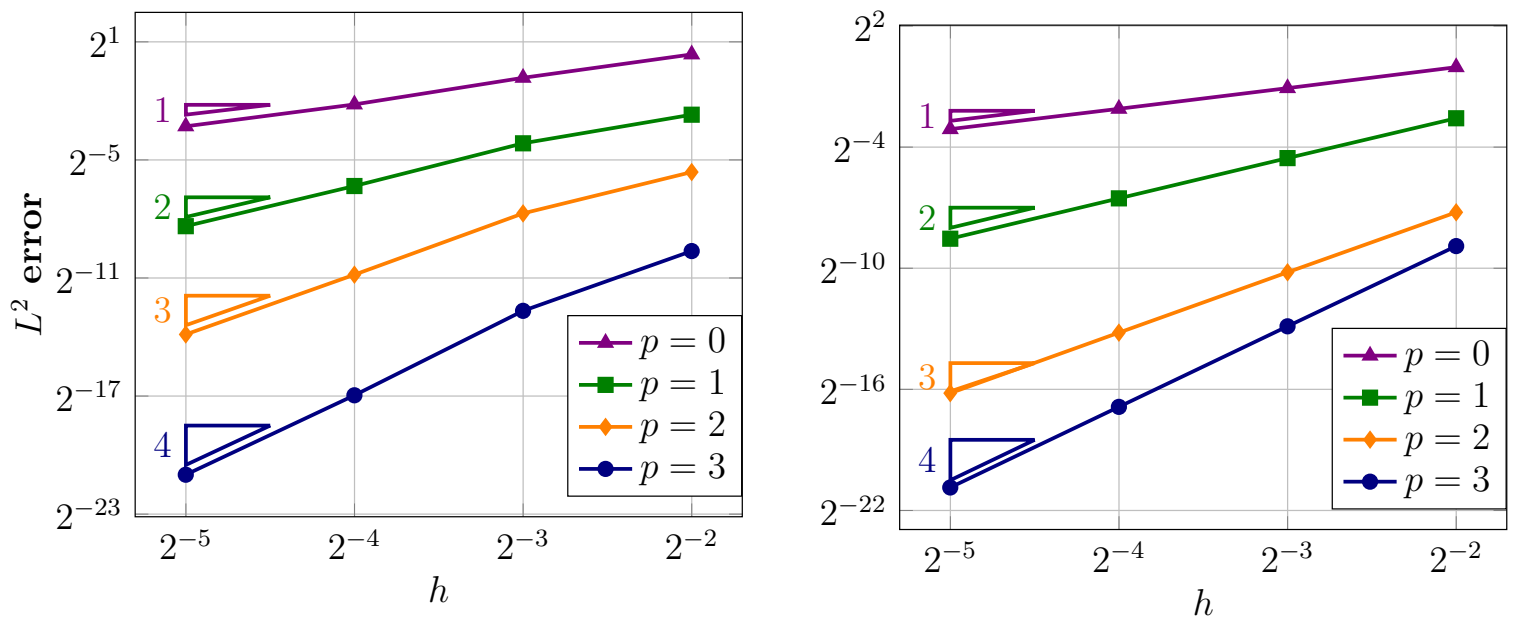

Figure 5.4. Convergence rates for $\left\|u-u_{h}\right\|$ on triangular meshes using Technique 1 on the left, and on rectangular meshes using Technique 1 on the right

5.5.4. Adaptivity. Let $\Omega=(0,1)^{2}$. We consider the same model problem (5.1.1) but now with zero sources $f=g=0$ and the non-zero initial condition

$$
\left.\mu\right|_{t=0}=-\phi_{0},\left.\quad q\right|_{t=0}=\phi_{0}
$$

in place of (5.1.1c), where $\phi_{0}=\exp \left(-1000\left((x-0.5)^{2}\right)\right)$. The boundary condition $\mu=0$ continues to remain the same. This simulates a beam reflecting off the Dirichlet boundary.

In Figure 5.3, we display a few iterates from the standard adaptive refinement algorithm using $p=3$ and the DPG error estimator. We started with the extremely coarse mesh shown in Figure 5.3(a), used the element-wise norms of $e_{h}$ to compute the DPG element error indicator, marked elements with more than $50 \%$ of the total indicated error, refined the marked elements (and more for conformity) by bisection, and repeated this adaptivity loop. The few iterates from the adaptivity loop shown in Figure 5.3 show the potential of the spacetime DPG method to easily capture localized features in spacetime. 
5.5.5. Convergence rates in three-dimensional spacetime. On $\Omega=(0,1)^{3}$, we consider the problem where the exact solution to the second order wave equation is given by $\phi(x, t)=\sin (\pi x) \sin (\pi y) t^{2}$. This corresponds to

$$
u=\left[\begin{array}{c}
c \pi \cos (\pi x) \sin (\pi y) t^{2} \\
c \pi \cos (\pi y) \sin (\pi x) t^{2} \\
2 \sin (\pi x) \sin (\pi y) t
\end{array}\right]
$$

$f=\sin (\pi x) \sin (\pi y)\left(2+2 c^{2} \pi^{2} t^{2}\right)$ and $g=0$.

In Figure 5.5, we show the convergence rates of $u_{h}$ for successively refined tetrahedral meshes, obtained using Technique 2 for $p=0,1,2,3$ to the right it shows analogous results obtained for successively refined hexahedral meshes using Technique 1. In all these cases, we observe $O\left(h^{p+1}\right)$ convergence rates for $u_{h}$.
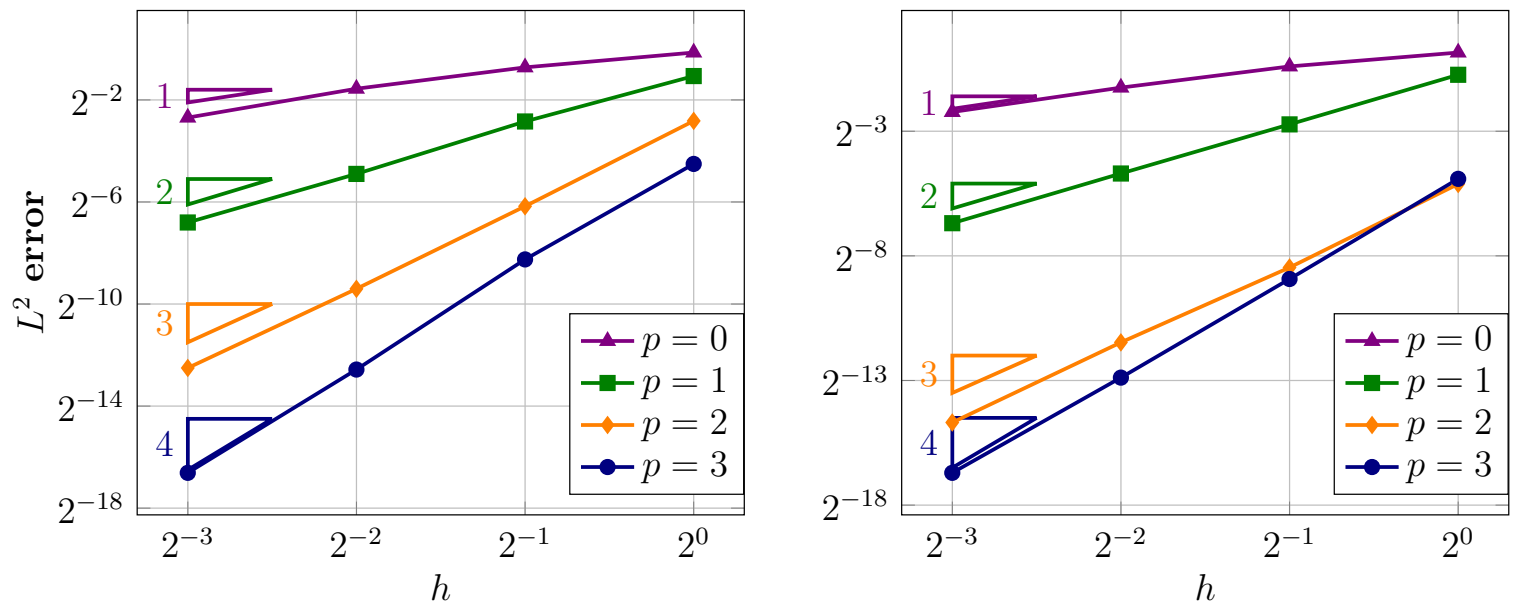

Figure 5.5. Convergence rates for $\left\|u-u_{h}\right\|$ on tretrahedral meshes using Technique 2 on the left, and on hexahedral meshes using Technique 1 on the right 


\section{Conclusions}

We have developed a framework providing sufficient conditions for the wellposedness of certain weak formulations of boundary value problems arising from linear spacetime partial differential equations in bounded domains. This includes not only first order formulations, as in Friedrichs systems, but also higher order partial differential equations. These ideas are essential for the proof of the wellposedness of the ultraweak formulations for evolution operators on spacetime cylinders (Chapters 4 and 5), as well as on spacetime tents (Chapter 2 and Chapter 3). We then studied the application of such formulations in spacetime DPG methods as well as in tent pitching schemes.

When studying the space of functions related to advective problems on domains where the inflow and outflow parts meet, we observed that traces of these functions satisfy a weak continuity property. We designed a new explicit finite element scheme of the tent pitching type for the wave equation that conforms to this weak continuity property for the lowest order case.

One of the contributions in this work is the proof of density of smooth functions that satisfy certain boundary conditions in a subspace of the graph space. These subspaces satisfy the boundary conditions only in a weak sense. It turns out that for hyperbolic problems in tent-shaped domains we have an exact characterization of these spaces through a trace lemma (see 2.4.1), for these spaces. Using this fact, it is easy to prove that a density result holds. In the case of a hyper-rectangular domain, we construct an extension operator that commutes with the spacetime operator such as 
the Schrödinger and wave operator, which allowed us to prove that the extensions are in graph space of the extension, and such that the smooth functions after convolution satisfy the corresponding boundary conditions.

The formulations we considered are applicable to multidimensional problems. In Chapter 5, numerical results confirmed the predicted optimal convergence rates of the DPG formulation for the case of the wave equation. A few adaptive iterates for the spacetime wave equation show the potential of the spacetime method to easily capture localized features in spacetime meshes.

Some future works that could build off those presented in this dissertation could include the study of other finite element methods, such as the the Constrained First Order System Least Squares (CFOSLS) method. Other future directions include the use of parallel techniques and fast solvers for spacetime formulations. 


\section{References}

[1] R. Abedi, M.A. Hawker, R.B. Haber, and K. Matous. An adaptive space-time discontinuous Galerkin method for cohesive models of elastodynamic fracture. Internat. J. Numer. Methods Eng., 81:12071241, 2010.

[2] R. A. Adams, and J. J. F. Fournie. Sobolev spaces, Elsevier Science, Oxford. Pure and Applied Mathematics, Vol. 140, 2003.

[3] G. P. Agrawal. Nonlinear Fiber Optics, fifth Edition. Academic Press, Waltham, Massachusetts, USA, 2012.

[4] J. H. Argyris and D.W. Scharpf. Finite Elements in Time and Space, Nuclear Engineering and Design, 10: 456-464, 1969.

[5] C. I. Bajer. Triangular and tetrahedral space-time finite elements in vibration analysis, International Journal for numerical methods in engineering, 23 (1986), pp. 2031-2048.

[6] J. Bramwell, L. Demkowicz, J. Gopalakrishnan and W. Qiu. A locking-free hp DPG method for linear elasticity with symmetric stresses. Numer. Math., 122(4):671-707, 2012.

[7] H. Brezis. Functional analysis, Sobolev spaces and Partial Differential Equations. Universitext, Springer, 2011.

[8] T. Bui-Thanh, L. Demkowicz and O. Ghattas. A unified discontinuous PetrovGalerkin method and its analysis for Friedrich's systems, SIAM Journal on Numerical Analysis, 51:1933-1958, 2013. 
[9] C. Carstensen, L. Demkowicz and J. Gopalakrishnan. Breaking spaces and forms for the DPG method and applications including Maxwell equations. Computers and Mathematics with Applications, 72, no. 3, 494-522, 2016.

[10] C. Carstensen, L. F. Demkowicz and J. Gopalakrishnan. A posteriori error control for DPG methods. SIAM J. Numer. Anal., 52:1335-1353, 2014.

[11] C. Carstensen, L. Demkowicz and J. Gopalakrishnan. DPG methods for Maxwell equations. In Susanne C. Brenner, Carsten Carstensen, Leszek Demkowicz, and Peter Wriggers, editors, Oberwolfach Reports: Computational Engineering, volume 43/2015, September 2015.

[12] T. Cazenave and A. Haraux. An Introduction to Semilinear Evolution Equations, Oxford Lecture Ser. Mah. Appl. 13, The Clarendon Press, Oxford University Press, New York, 1998; translated from the 1990 French original by Yvan Martel and revised by the authors.

[13] P. G. Ciarlet. The Finite Element Method for Elliptic Problems, North-Holland Publishing Company, Amsterdam, 1978.

[14] R. Courant, K. Friedrichs and H. Lewy. Über die partiellen Differenzengleichungen der mathematischen Physik, Math. Ann., 100: 32-74, 1928.

[15] W. Dahmen, C. Huang, C. Schwab and G. Welper. Adaptive Petrov-Galerkin methods for 1st order transport equations. SIAM J. Numer. Anal., 50:2420-2445, 2012.

[16] L. Demkowicz, S. Nagaraj, J. Gopalakrishnan and P. Sepúlveda. A spacetime DPG method for the Schrödinger equation. SIAM Journal on Numerical Analysis, Vol. 55, No. 4, pp. 1740-1759, 201.7

[17] L. Demkowicz and J. Gopalakrishnan. A class of discontinuous Petrov-Galerkin methods. Part I: The transport equation. Computer Methods in Applied Mechanics and Engineering, 199:1558-1572, 2010. 
[18] L. Demkowicz and J. Gopalakrishnan. Analysis of the DPG method for the Poisson equation. SIAM J. Numer. Anal., 49:1788-1809, 2011.

[19] L. Demkowicz and J. Gopalakrishnan. A class of discontinuous Petrov-Galerkin methods. Part II: Optimal test functions. Numerical Methods for Partial Differential Equations, 27:70-105, 2011.

[20] L. Demkowicz and J. Gopalakrishnan An overview of the discontinuous Petrov Galerkin method, in Recent Developments in Discontinuous Galerkin Finite Element Methods for Partial Differential Equations: 2012 John H Barret Memorial Lectures, X. Feng, O. Karakashian, and Y. Xing, eds., vol. 157 of The IMA Volumes in Mathematics and its Applications, Institute for Mathematics and its Applications, Minneapolis, Springer, pp. 149-180, 2013.

[21] L. Demkowicz, J. Gopalakrishnan, I. Muga and J. Zitelli. Wavenumber explicit analysis for a DPG method for the multidimensional Helmholtz equation. Computer Methods in Applied Mechanics and Engineering, 213/216:126-138, March 2012.

[22] L. Demkowicz, J. Gopalakrishnan, A.H. Niemi. A class of discontinuous PetrovGalerkin methods. Part III: Adaptivity. Appl. Numer. Math. 62 , no. 4, 396427. $65 \mathrm{~N} 30$ (65N50), 2012.

[23] L. Demkowicz and N. Heuer. Robust DPG method for convection-dominated diffusion problems. SIAM J. Numer. Anal., 51(5):2514-2537, 2013.

[24] D.A. Di Pietro and A. Ern. Mathematical Aspects of Discontinuous Galerkin Methods. Series: Mathematiques et Applications, Vol. 69, 2012.

[25] W. Dorfler, S.Findeisen, and C.Wierners Space-time Discontinuous Galerkin Discretizations for linear First-order hyperbolic evolution systems, Comput. Meth. in Appl. Math, Vol16, 409-428, 2016. 
[26] DPG Methods in NGSolve, https://github.com/jayggg/DPG. Software hosted on GitHub.

[27] T. E. Ellis, J.L. Chan and L. F. Demkowicz. Robust DPG methods for transient convection-diffusion. ICES Report, The Institute for Computational Engineering and Sciences, The University of Texas at Austin, 15-21, 2015.

[28] T. E. Ellis, L. F. Demkowicz, J. L. Chan and R. D. Moser. Space-time DPG: Designing a method for massively parallel CFD. ICES Report, The Institute for Computational Engineering and Sciences, The University of Texas at Austin, 1432,2014 .

[29] J. Erickson, D. Guoy, J. M. Sullivan and A. Üngör. Building spacetime meshes over arbitrary spatial domains, Engineering with Computers, 20: 342-353, 2005.

[30] A. Ern, J.-L. Guermond and G. Caplain. An intrinsic criterion for the bijectivity of Hilbert operators related to Friedrichs' systems, Comm. Partial Differential Equations, 32 : 317-341, 2007.

[31] J. Ernesti and C. Wieners. A space-time DPG method for acoustic waves. Submitted.

[32] L. C. Evans. Partial Differential Equations. Graduate Studies in Mathematics vol. 19, American Mathematical Society, Providence, Rhode Island, USA, 1998.

[33] R. S. Falk and G. R. Richter. Explicit finite element methods for symmetric hyperbolic equations, SIAM J. Numer. Anal., 36 : 935-952, 1999 (electronic).

[34] T. Führer, N. Heuer and J. Sen Gupta. A time-stepping DPG scheme for the heat equation. Comput. Methods Appl. Math. 17 (2), 237-253, 2017.

[35] F. Fuentes, B. Keith, L. F. Demkowicz and S. Nagaraj. Orientation embedded high order shape functions for the exact sequence elements of all shapes. Computers and Mathematics with Applications, 70:353-458, 2015. 
[36] K. O. Friedrichs. Symmetric positive linear differential equations, Comm. Pure Appl. Math., 11: 333-418, 1958.

[37] J. Gopalakrishnan and P. Sepúlveda. A spacetime DPG method for acoustic waves, in review, 2018.

[38] J. Gopalakrishnan and W. Qiu. An analysis of the practical DPG method. Math. Comp., 83:537-552, 2014.

[39] J. Gopalakrishnan, J. Schöberl and C. Wintersteiger. Mapped tent pitching schemes for hyperbolic systems, SIAM Journal on Scientific Computing, Vol. 39, No. 6, pp. B1043-B1063, 2017.

[40] J. Gopalakrishnan, P. Monk and P. Sepúlveda. A tent pitching scheme motivated by Friedrichs theory, Computers and Mathematics with Applications, 70:1114$1135,2015$.

[41] B. Gustafsson. High order difference methods for time dependent PDE, of Springer Series in Computational Mathematics, Springer-Verlag, Berlin, vol 38, 2008.

[42] M. Jensen. Discontinuous Galerkin Methods for Friedrichs Systems with Irregular Solutions. PhD thesis, University of Oxford, 2004.

[43] Kato, Tosio, Chapter 5. Operators in Hilbert Space, Perturbation theory for linear operators. Classics in Mathematics, Springer-Verlag, 1995.

[44] Z. Kaczkowski, The method of finite space-time elements in dynamics of structures, Journal of Technical Pysics, 16 , pp. 69-84, 1975.

[45] B. Keith, F. Fuentes and L. F. Demkowicz. The DPG methodology applied to different variational formulations of linear elasticity. Computer Methods in Applied Mechanics and Engineering, 309:579-609, 2016.

[46] S. Kesavan. Topics in Functional Analysis and Applications Wiley Eastern Limited,1989. 
[47] R. J. LeVeque. Finite volume methods for hyperbolic problems, Cambridge Texts in Applied Mathematics, Cambridge University Press, Cambridge, 2002.

[48] R. B. Lowrie, P. L. Roe and B. Van Leer. A space-time discontinuous Galerkin method for the time-accurate numerical solution of hyperbolic conservation laws, in Proceedings of the 12th AIAA Computational Fluid Dynamics Conference, no. 95-1658, 1995.

[49] S. T. Miller and R. B. Haber, A spacetime discontinuous Galerkin method for hyperbolic heat conduction, Computer Methods in Applied Mechanics and Engineering, 198 (2008), pp. 194-209.

[50] P. Monk and G. R. Richter. A discontinuous Galerkin method for linear symmetric hyperbolic systems in inhomogeneous media, J. Sci. Comput., 22/23: 443-477, 2005.

[51] M. Neumüeller. Space-time Methods: Fast Solvers and Applications, PhD thesis, Graz University of Technology, 2013.

[52] M. Neumüeller, P. S. Vassilevski and U. Villa. Space-time CFOSLS Methods with AMGe Upscaling, 23th International Conference on Domain Decomposition Methods Jeju Island, South Korea, July 6, 2015 through July 10, , 2015.

[53] J. T. Oden. A general theory of finite elements II. Applications, International Journal for Numerical Methods in Engeneering, 1 : 247-259, 1969.

[54] J. T. Oden and L. Demkowicz. Applied Functional Analysis. CRC Press, 2010.

[55] J. Palaniappan, R. B. Haber and R. L. Jerrard, A spacetime discontinuous Galerkin method for scalar conservation laws, Computer Methods in Applied Mechanics and Engineering, 193 (2004), pp. 3607-3631.

[56] G. R. Richter. An explicit finite element method for the wave equation. Appl. Numer. Math., 16:65-30, 1994. A Festschrift to honor Professor Robert Vichnevetsky on his 65 th birthday. 
[57] R. D. Richtmyer and K. W. Morton. Difference methods for initial-value problems, Robert E. Krieger Publishing Co., Inc., Malabar, FL, second ed., 1994.

[58] N. V. Roberts, T. Bui-Thanh and L. F. Demkowicz. The DPG Method for the Stokes Problem. Computers and Mathematics with Applications, 67:966-995, 2014.

[59] J. Schöberl. Netgen. https://sourceforge.net/projects/netgen-mesher/

[60] —. NGSolve, Sofware hosted. https://ngsolve.org.

[61] M. Renardy and R. C. Rogers. An introduction to partial differential equations, Springer Science \& Business Media, 13, 2006.

[62] C. Schwab and R. Stevenson, Space-time adaptive wavelent methods for parabolic evolution problems, Math. Comp. 78, 1293-1318, 2009.

[63] J. K. Shaw. Mathematical Principles of Optical Fiber Communication. CBMSNSF Regional Conference Series in Applied Mathematics (76). SIAM: Society for Industrial and Applied Mathematics, 2004.

[64] O. Steinbach, Space-time finite element methods for parabolic problems, Comput. Methods Appl. Math. 15, 551-566, 2015.

[65] J. C. Strikwerda. Finite difference schemes and partial differential equations, The Wadsworth \& Brooks/Cole Mathematics Series, Wadsworth \&3 Brooks/Cole Advanced Books \& Software, Pacific Grove, CA, 1989.

[66] T. Tao. Nonlinear Dispersive Equations. CBMS Regional Conference Series in Mathematics vol. 106, Published for the Conference Board of the Mathematical Sciences, Washington, DC by the American Mathematical Society, 2006.

[67] A. Üngör and A. Sheffer. Pitching tents in space-time: mesh generation for discontinuous Galerkin method, Internat. J. Found. Comput. Sci., 13: 201-221, 2002.

[68] C. Wieners. The skeleton reduction for finite element substructuring methods. ENUMATH 2015 Proceedings, 2016. 
[69] C. Wieners and B. Wohlmuth. Robust operator estimates and the application to substructuring methods for first-order systems, ESAIM: Mathematical Modelling and Numerical Analysis, 48, 1473-1494, 2014.

[70] K. Yee. Numerical solution of initial boundary value problems involving maxwell's equations in isotropic media, IEEE Trans. Antennas and Prop., 14: 302-307, 1966.

[71] L. Yin, A. Acharia, N. Sobh, R. B. Haber, and D. A. Tortorelli, A spacetime discontinuous Galerkin method for elastodynamics analysis, in Discontinuous Galerkin Methods: Theory, Computation and Applications, B. Cockburn and G. Karniadakis and C. W. Shu(eds), Springer Verlag, pp. 459-464, 2000. 